Supporting Information

\title{
Isocyanides synthesis by reacting primary amines with difluorocarbene
}

\author{
Yi-Xin Si, Peng-Fei Zhu and Song-Lin Zhang* \\ Key Laboratory of Synthetic and Biological Colloids, Ministry of Education, School of Chemical \\ and Material Engineering, Jiangnan University, Wuxi 214122, Jiangsu, China \\ E-mail: slzhang@jiangnan.edu.cn
}

Table of contents

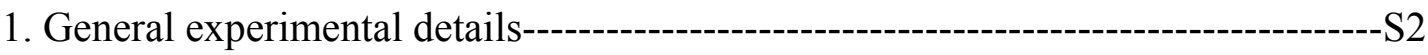

2. General procedure for isocyanides synthesis from primary amines-------------------S3

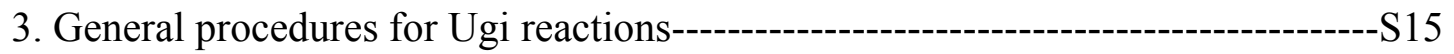

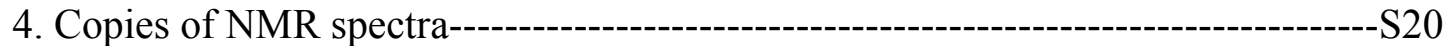




\section{General experimental details}

All of the chemicals were purchased commercially, and used as received without further purification. All of the reactions were performed in a sealed Schlenk tube under $\mathrm{N}_{2}$ atmosphere which was realized through evacuation/backfill techniques after three times. The reactions were monitored by TLC analysis with stains visualized under UV irradiation until the amine substrates were completely consumed. Column chromatography on silica gel was used to obtain purified products that are suitable for NMR spectroscopic characterization. NMR spectra were recorded on a $400 \mathrm{MHz}$ spectrometer for ${ }^{1} \mathrm{H} \mathrm{NMR}, 101 \mathrm{MHz}$ for ${ }^{13} \mathrm{C} \mathrm{NMR}$, and $376 \mathrm{MHz}$ for ${ }^{19} \mathrm{~F}$ NMR. Chemical shifts are reported in ppm and referenced to residual solvent peaks $\left({ }^{1} \mathrm{H}\right.$ and ${ }^{13} \mathrm{C}$ are referenced to $\mathrm{HCCl}_{3} ;{ }^{19} \mathrm{~F}$ are relative to $\mathrm{CFCl}_{3}$ ). NMR signals are reported as follows to delineate possible splitting: s, singlet; br s, broad singlet; d, doublet; t, triplet; q, quartet; and m, multiplet. Coupling constants are reported in Hertz where present. All the ${ }^{13} \mathrm{C}$ and ${ }^{19} \mathrm{~F}$ NMR spectra were obtained with proton decoupling. Elemental analyses were performed by the Analytic Laboratory of Jiangnan University. High resolution mass spectra (HRMS) were determined on Thermo Scientific LTQ Orbitrap XL with ESI ionization mode. FT-IR spectra were recorded on an IRTracer-100 spectrometer. 


\section{General procedure for isocyanides synthesis from primary amines}

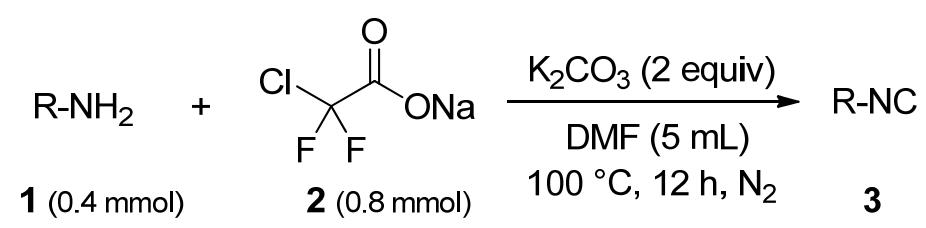

In an oven-dried 25-mL Schlenk tube equipped with a magnetic stir bar were added a primary amine $\mathrm{R}-\mathrm{NH}_{2}(\mathbf{1})(0.4 \mathrm{mmol})$, sodium chlorodifluoroacetate (2) $(0.8$ mmol) and $\mathrm{K}_{2} \mathrm{CO}_{3}(0.8 \mathrm{mmol})$. The Schlenk tube was evacuated and refilled with dry nitrogen. Dry DMF (5 mL) was then added by syringe. The contents in the tube were vigorously stirred for 12 hours at $100{ }^{\circ} \mathrm{C}$ (heated in an oil bath). The reaction mixture was then allowed to cool to room temperature. The resulting mixture was extracted by dichloromethane. The combined organic layers were washed with a large amount of water for 4 times, with brine for once and then dried over magnesium sulfate. The solvent was removed under vacuum and the residuals were purified by column chromatography on silica using a mixture of petroleum ether (PE) and ethyl acetate (EA) as the eluent to give purified isocyanide compound 3.<smiles></smiles>

1-isocyano-4-methoxybenzene (3a; $37 \mathrm{mg}, 70 \%$ ). Eluted with petroleum ether/ethyl acetate $=20: 1(\mathrm{v} / \mathrm{v})$. Yellow solid; melting point: $29-32{ }^{\circ} \mathrm{C} .{ }^{1} \mathrm{H}$ NMR $(400 \mathrm{MHz}$, $\left.\mathrm{CDCl}_{3}\right) \delta 7.29(\mathrm{~d}, J=8.9 \mathrm{~Hz}, 2 \mathrm{H}), 6.86(\mathrm{~d}, J=9.0 \mathrm{~Hz}, 2 \mathrm{H}), 3.81(\mathrm{~s}, 3 \mathrm{H}) .{ }^{13} \mathrm{C} \mathrm{NMR}$ $\left(101 \mathrm{MHz}, \mathrm{CDCl}_{3}\right) \delta 162.6(\mathrm{t}, J=5.7 \mathrm{~Hz}), 159.9$ (s), $127.8(\mathrm{~s}), 119.5$ (t, $\left.J=14.0 \mathrm{~Hz}\right)$, 114.6 (s), $55.6(\mathrm{~s})$. FT-IR $\left(v_{\max }, \mathrm{cm}^{-1}\right): 2962,2123$ (vs, $\mathrm{N}=\mathrm{C}$ stretching vibration), $1606,1506,1300,1192,1106,1027$.

These data are in good agreement with literature report. ${ }^{\mathrm{S} 1}$

Scale-up experiment using 4 mmol 1a (para-methoxyaniline): Following the general procedure using $4 \mathrm{mmol} \mathrm{1a}, 8 \mathrm{mmol}$ 2, the desired 3a was obtained in $324 \mathrm{mg}, 61 \%$ yield. 
$\overbrace{}^{N C}$

4-isocyano- $N, N$-dimethylaniline(3b; $42 \mathrm{mg}, 72 \%$ ). Eluted with petroleum ether/ethyl acetate $=20: 1(\mathrm{v} / \mathrm{v})$. White solid; melting point: $60-62{ }^{\circ} \mathrm{C} .{ }^{1} \mathrm{H} \mathrm{NMR}\left(400 \mathrm{MHz}, \mathrm{CDCl}_{3}\right)$ $\delta 7.22(\mathrm{~d}, J=8.9 \mathrm{~Hz}, 2 \mathrm{H}), 6.59(\mathrm{~d}, J=9.1 \mathrm{~Hz}, 2 \mathrm{H}), 2.99(\mathrm{~s}, 6 \mathrm{H}) .{ }^{13} \mathrm{C} \mathrm{NMR}(101 \mathrm{MHz}$, $\left.\mathrm{CDCl}_{3}\right) \delta 159.7$ - $159.4(\mathrm{~m}), 148.7(\mathrm{~s}), 125.7(\mathrm{~s}), 113.5-113.1(\mathrm{~m}), 110.0(\mathrm{~s}), 38.6(\mathrm{~s})$. FT-IR $\left(v_{\max }, \mathrm{cm}^{-1}\right): 2901,2818,2118,1883,1909,1524,1447,1368,1172,1123$, $1066,945,819,516$.

These data are in good agreement with literature report. ${ }^{\mathrm{S} 1}$<smiles>O=[N+]([O-])c1ccc(O)cc1</smiles>

4-isocyanophenol (3c; $20 \mathrm{mg}, 42 \%)$. Eluted with petroleum ether/ethyl acetate $=4: 1$ (v/v). White solid; ${ }^{1} \mathrm{H}$ NMR (400 MHz, $\left.\mathrm{CDCl}_{3}\right) \delta 7.26(\mathrm{~d}, J=8.6 \mathrm{~Hz}, 2 \mathrm{H}), 6.83(\mathrm{~d}, J=$ $8.8 \mathrm{~Hz}, 2 \mathrm{H}), 6.10(\mathrm{~s}, 1 \mathrm{H}) .{ }^{13} \mathrm{C} \mathrm{NMR}\left(101 \mathrm{MHz}, \mathrm{CDCl}_{3}\right) \delta 161.4(\mathrm{~m}), 156.5(\mathrm{~s}), 128.0$ (s), $119.4(\mathrm{~m}), 116.2(\mathrm{~s})$. FT-IR $\left(v_{\max }, \mathrm{cm}^{-1}\right): 3281,2925,2123,1608,1508,1447$, 1224, 836. HRMS (ESI) m/z calcd for $\mathrm{C}_{7} \mathrm{H}_{6} \mathrm{NO}^{+}(\mathrm{M}+\mathrm{H})^{+} 120.0449$, found 120.0445 .<smiles>Nc1ccc([N+](=O)[O-])cc1</smiles>

4-isocyanoaniline (3d; $39 \mathrm{mg}, 83 \%$ ). Eluted with petroleum ether/ethyl acetate $=4: 1$ (v/v). White solid; melting point: $64-66{ }^{\circ} \mathrm{C} .{ }^{1} \mathrm{H}$ NMR $\left(400 \mathrm{MHz}, \mathrm{CDCl}_{3}\right) \delta 7.14(\mathrm{~d}, J=$ $8.4 \mathrm{~Hz}, 2 \mathrm{H}), 6.58(\mathrm{~d}, J=8.7 \mathrm{~Hz}, 2 \mathrm{H}), 3.94(\mathrm{~s}, 2 \mathrm{H}) .{ }^{13} \mathrm{C} \mathrm{NMR}\left(101 \mathrm{MHz}, \mathrm{CDCl}_{3}\right) \delta$ $161.3(\mathrm{~m}), 147.3(\mathrm{~s}), 127.7(\mathrm{~s}), 117.1(\mathrm{t}, J=14.4 \mathrm{~Hz}), 114.8(\mathrm{~s})$. FT-IR $\left(v_{\max }, \mathrm{cm}^{-1}\right)$ : $3462,3357,3221,2123,1625,1603,1508,1298,1196,1163,828,515$.

These data are in good agreement with literature report. ${ }^{\mathrm{S} 1}$<smiles></smiles> 
1-isocyano-4-phenoxybenzene (3e; $40 \mathrm{mg}, 51 \%)$. Eluted with petroleum ether/ethyl acetate $=10: 1(\mathrm{v} / \mathrm{v})$. Yellow oil; ${ }^{1} \mathrm{H}$ NMR $\left(400 \mathrm{MHz}, \mathrm{CDCl}_{3}\right) \delta 7.42-7.36(\mathrm{~m}, 2 \mathrm{H})$, $7.33(\mathrm{~d}, J=8.9 \mathrm{~Hz}, 2 \mathrm{H}), 7.19$ (t, $J=7.4 \mathrm{~Hz}, 1 \mathrm{H}), 7.03$ (d, $J=7.6 \mathrm{~Hz}, 2 \mathrm{H}), 6.96$ (d, $J=$ $9.0 \mathrm{~Hz}, 2 \mathrm{H}) .{ }^{13} \mathrm{C} \mathrm{NMR}\left(101 \mathrm{MHz}, \mathrm{CDCl}_{3}\right) \delta 163.5$ (m), 158.3 (s), 155.7 (s), 130.1 (s), $128.0(\mathrm{~s}), 124.6(\mathrm{~s}), 121.2(\mathrm{t}, J=13.3 \mathrm{~Hz}), 119.8(\mathrm{~s}), 118.6(\mathrm{~s})$. FT-IR $\left(v_{\max }, \mathrm{cm}^{-1}\right)$ : 2123, 1508, 1499, 1438, 1244, 1101, 1022, 871, 801, 691. Anal. Calcd for $\mathrm{C}_{13} \mathrm{H}_{9} \mathrm{NO}$ : C, 79.98; H, 4.65; N, 7.17. Found: C, 80.12; H, 4.72; N, 7.06.<smiles>N#[N+]c1ccc(Oc2ccc([N+]=[N+]=[N-])cc2)cc1</smiles>

4,4'-oxybis(isocyanobenzene) (3f; $17 \mathrm{mg}, 20 \%$ ). Eluted with petroleum ether/ethyl acetate $=20: 1(\mathrm{v} / \mathrm{v})$. White solid; melting point: $144-146{ }^{\circ} \mathrm{C} .{ }^{1} \mathrm{H}$ NMR $(400 \mathrm{MHz}$, $\left.\mathrm{CDCl}_{3}\right) \delta 7.39(\mathrm{~d}, J=8.9 \mathrm{~Hz}, 4 \mathrm{H}), 7.01(\mathrm{~d}, J=9.0 \mathrm{~Hz}, 4 \mathrm{H}) .{ }^{13} \mathrm{C}$ NMR $(101 \mathrm{MHz}$, $\left.\mathrm{CDCl}_{3}\right) \delta 164.35$ - $164.16(\mathrm{~m}), 156.7$ (s), $128.3(\mathrm{~s}), 122.5$ - $122.3(\mathrm{~m}), 119.7$ (s). FT-IR $\left(v_{\max }, \mathrm{cm}^{-1}\right): 3441,2962,2125,1592,1492,1295,1186,1097,879,837,800$.

These data are in good agreement with literature report. ${ }^{\mathrm{S} 2}$<smiles>Nc1ccc(Oc2ccc([N+](=O)[O-])cc2)cc1</smiles>

4-(4-isocyanophenoxy)aniline (3g; $34 \mathrm{mg}, 40 \%$ ). Eluted with petroleum ether/ethyl acetate $=10: 1(\mathrm{v} / \mathrm{v})$. Yellow solid; ${ }^{1} \mathrm{H}$ NMR $\left(400 \mathrm{MHz}, \mathrm{CDCl}_{3}\right) \delta 7.30(\mathrm{~d}, J=9.0 \mathrm{~Hz}$, 2H), 6.89 (t, $J=8.3 \mathrm{~Hz}, 4 \mathrm{H}), 6.72(\mathrm{~d}, J=8.7 \mathrm{~Hz}, 2 \mathrm{H}), 3.54(\mathrm{br} \mathrm{s}, 2 \mathrm{H}) .{ }^{13} \mathrm{C}$ NMR $(101$ $\left.\mathrm{MHz}, \mathrm{CDCl}_{3}\right) \delta 162.9(\mathrm{~m}), 159.7$ (s), 147.1 (s), 143.7 (s), 127.8 (s), 121.6 (s), 120.4 (m), 117.2 (s), 116.3 (s). FT-IR ( $\left.v_{\max }, \mathrm{cm}^{-1}\right)$ : 3355, 3221, 2960, 2125, 1608, 1493, 1241, 1197, 1103, 834. Anal. calcd. for $\mathrm{C}_{13} \mathrm{H}_{10} \mathrm{~N}_{2} \mathrm{O}: \mathrm{C}, 74.27 ; \mathrm{H}, 4.79 ; \mathrm{N}, 13.33$. Found: C, 74.40; H, 4.85; N, 13.20 .<smiles>N#Cc1ccc(Nc2ccccc2)cc1</smiles> 
4-isocyano-N-phenylaniline (3h; $47 \mathrm{mg}, 61 \%$ ). Eluted with petroleum ether/ethyl acetate $=20: 1(\mathrm{v} / \mathrm{v})$. White solid; melting point: $71-73{ }^{\circ} \mathrm{C} .{ }^{1} \mathrm{H}$ NMR $\left(400 \mathrm{MHz}, \mathrm{CDCl}_{3}\right)$ $\delta 7.33(\mathrm{t}, J=7.9 \mathrm{~Hz}, 2 \mathrm{H}), 7.23(\mathrm{~d}, J=8.8 \mathrm{~Hz}, 2 \mathrm{H}), 7.12(\mathrm{~d}, J=7.5 \mathrm{~Hz}, 2 \mathrm{H}), 7.05$ (t, $J$ $=7.4 \mathrm{~Hz}, 1 \mathrm{H}), 6.96(\mathrm{~d}, J=8.9 \mathrm{~Hz}, 2 \mathrm{H}), 5.92(\mathrm{br} \mathrm{s}, 1 \mathrm{H}) .{ }^{13} \mathrm{C} \mathrm{NMR}\left(101 \mathrm{MHz}, \mathrm{CDCl}_{3}\right)$ $\delta 162.0$ (t, $J=8.1 \mathrm{~Hz}), 144.7$ (s), 141.1 (s), 129.6 (s), 127.7 (s), 123.0 (s), 119.9 (s), $118.3(\mathrm{t}, J=14.4 \mathrm{~Hz}), 116.0$ (s). FT-IR $\left(v_{\max }, \mathrm{cm}^{-1}\right): 3323,2961,2120,1593,1512$, 1495, 1323, 1260, 1163, 1078, 1026, 808, 758, 693, 515. HRMS (ESI) m/z calcd for $\mathrm{C}_{13} \mathrm{H}_{11} \mathrm{~N}_{2}^{+}(\mathrm{M}+\mathrm{H})^{+}$195.0922, found 195.0919.<smiles>CC(=O)Nc1ccc([N+](=O)[O-])cc1</smiles>

$N$-(4-isocyanophenyl)acetamide (3i; $26 \mathrm{mg}, 41 \%)$. Eluted with petroleum ether/ethyl acetate $=5: 1(\mathrm{v} / \mathrm{v})$. White solid; melting point: $182-185{ }^{\circ} \mathrm{C} .{ }^{1} \mathrm{H}$ NMR $(400 \mathrm{MHz}$, DMSO-d $\left.^{6}\right) \delta 10.24$ (br s, 1H), $7.68(\mathrm{~d}, J=8.9 \mathrm{~Hz}, 2 \mathrm{H}), 7.49$ (d, $\left.J=8.9 \mathrm{~Hz}, 2 \mathrm{H}\right), 2.07$ (s, 3H). ${ }^{13} \mathrm{C}$ NMR (101 MHz, DMSO-d $\left.{ }^{6}\right) \delta 169.3(\mathrm{~s}), 163.6$ - $163.3(\mathrm{~m}), 140.9$ (s), $127.5(\mathrm{~s}), 119.8(\mathrm{~s}), 119.1$ - $118.9(\mathrm{~m}), 24.5(\mathrm{~s})$. FT-IR $\left(v_{\max }, \mathrm{cm}^{-1}\right): 3648,3446,3299$, $2124,1667,1602,1555,1504,1408,1331,1265,831$.

These data are in good agreement with literature report. ${ }^{\mathrm{S} 3}$<smiles>CSc1ccc([N+](C)(C)C)cc1</smiles>

(4-isocyanophenyl)(methyl)sulfane (3j; $19 \mathrm{mg}, 32 \%$ ). Eluted with petroleum ether/ethyl acetate $=20: 1(\mathrm{v} / \mathrm{v})$. Yellow solid; ${ }^{1} \mathrm{H}$ NMR $\left(400 \mathrm{MHz}, \mathrm{CDCl}_{3}\right) \delta 7.28(\mathrm{~d}, J$ $=8.6 \mathrm{~Hz}, 2 \mathrm{H}), 7.21(\mathrm{~d}, J=8.7 \mathrm{~Hz}, 2 \mathrm{H}), 2.49(\mathrm{~s}, 3 \mathrm{H}) .{ }^{13} \mathrm{C} \mathrm{NMR}\left(101 \mathrm{MHz}, \mathrm{CDCl}_{3}\right) \delta$ 164.3 - 164.0 (m), 141.3 (s), 126.7 (s), 126.4 (s), 123.3 - 122.9 (m), 15.3 (s). FT-IR $\left(v_{\max }, \mathrm{cm}^{-1}\right): 3439,2962,2121,1593,1488,1436,1403,1260,1198,1091,1015,819$, 512.

These data are in good agreement with literature report. ${ }^{\mathrm{S} 1}$ 
$\overbrace{}^{N C}$

1-ethoxy-4-isocyanobenzene (3k; $39 \mathrm{mg}, 67 \%$ ). Eluted with petroleum ether/ethyl acetate $=20: 1(\mathrm{v} / \mathrm{v})$. Yellow oil; ${ }^{1} \mathrm{H}$ NMR $\left(400 \mathrm{MHz}, \mathrm{CDCl}_{3}\right) \delta 7.28(\mathrm{~d}, J=8.9 \mathrm{~Hz}$, $2 \mathrm{H}), 6.84(\mathrm{~d}, J=9.0 \mathrm{~Hz}, 2 \mathrm{H}), 4.03(\mathrm{q}, J=7.0 \mathrm{~Hz}, 2 \mathrm{H}), 1.41(\mathrm{t}, J=7.0 \mathrm{~Hz}, 3 \mathrm{H}) .{ }^{13} \mathrm{C}$ NMR (101 MHz, $\left.\mathrm{CDCl}_{3}\right) \delta 162.5(\mathrm{t}, J=5.1 \mathrm{~Hz}), 159.3(\mathrm{~s}), 127.7(\mathrm{~s}), 119.3(\mathrm{t}, J=$ $14.0 \mathrm{~Hz}), 115.0$ (s), 63.9 (s), 14.6 (s). FT-IR $\left(v_{\max }, \mathrm{cm}^{-1}\right): 2981,2930,2122,1606$, 1583, 1504, 1476, 1395, 1298, 1256, 1115, 1042, 837, 723, 524.

These data are in good agreement with literature report. ${ }^{\mathrm{S} 4}$<smiles>[N-]Cc1ccccc1</smiles>

(isocyanomethyl)benzene (3l; $30 \mathrm{mg}, 65 \%$ ). Eluted with petroleum ether/ethyl acetate $=20: 1(\mathrm{v} / \mathrm{v})$. Yellow oil; ${ }^{1} \mathrm{H}$ NMR $\left(400 \mathrm{MHz}, \mathrm{CDCl}_{3}\right) \delta 7.45-7.31(\mathrm{~m}, 5 \mathrm{H})$, 4.65 (br s, 2H). ${ }^{13} \mathrm{C}$ NMR (101 MHz, $\mathrm{CDCl}_{3}$ ) $\delta 157.7$ (t, $J=5.4 \mathrm{~Hz}$ ), 132.3 (s), 129.0 (s), $128.4(\mathrm{~s}), 126.6(\mathrm{~s}), 45.6(\mathrm{t}, J=7.2 \mathrm{~Hz})$. FT-IR $\left(v_{\max }, \mathrm{cm}^{-1}\right): 3726,3440,2961$, 2925, 2149, 1735, 1600, 1496, 1454, 1260, 1081, 1022, 799.

These data are in good agreement with literature report. ${ }^{\mathrm{S} 5}$<smiles>COc1ccc(CN)cc1</smiles>

1-(isocyanomethyl)-4-methoxybenzene (3m; $39 \mathrm{mg}, 67 \%$ ). Eluted with petroleum ether/ethyl acetate $=20: 1(\mathrm{v} / \mathrm{v})$. Colorless liquid; ${ }^{1} \mathrm{H}$ NMR $\left(400 \mathrm{MHz}, \mathrm{CDCl}_{3}\right) \delta 7.26$ $(\mathrm{d}, J=8.7 \mathrm{~Hz}, 2 \mathrm{H}), 6.92(\mathrm{~d}, J=8.7 \mathrm{~Hz}, 2 \mathrm{H}), 4.56$ (t, $J=1.9 \mathrm{~Hz}, 2 \mathrm{H}), 3.82(\mathrm{~s}, 3 \mathrm{H}) .{ }^{13} \mathrm{C}$ NMR (101 MHz, $\left.\mathrm{CDCl}_{3}\right) \delta 159.7$ (s), 157.1 (t, $\left.J=5.2 \mathrm{~Hz}\right), 128.2$ (s), $124.6(\mathrm{~s}), 114.4$ (s), $55.4(\mathrm{~s}), 45.1(\mathrm{t}, J=7.0 \mathrm{~Hz})$. FT-IR $\left(v_{\max }, \mathrm{cm}^{-1}\right): 3440,2961,2837,2148,1613$, 1514, 1441, 1302, 1254, 1178, 1032, 814, 574.

These data are in good agreement with literature report. ${ }^{\mathrm{S} 6}$ 
NC

1-fluoro-4-(isocyanomethyl)benzene (3n; $45 \mathrm{mg}, 83 \%$ ). Eluted with petroleum ether/ethyl acetate $=20: 1(\mathrm{v} / \mathrm{v})$. Yellow oil; ${ }^{1} \mathrm{H}$ NMR $\left(400 \mathrm{MHz}, \mathrm{CDCl}_{3}\right) \delta 7.33(\mathrm{dd}, J$ $=8.5,5.2 \mathrm{~Hz}, 2 \mathrm{H}), 7.09(\mathrm{t}, J=8.6 \mathrm{~Hz}, 2 \mathrm{H}), 4.61(\mathrm{~s}, 2 \mathrm{H}) .{ }^{13} \mathrm{C} \mathrm{NMR}\left(101 \mathrm{MHz}, \mathrm{CDCl}_{3}\right)$ $\delta 162.7(\mathrm{~d}, J=247.5 \mathrm{~Hz}), 158.0(\mathrm{t}, J=5.1 \mathrm{~Hz}), 128.5(\mathrm{~d}, J=8.3 \mathrm{~Hz}), 128.2(\mathrm{~d}, J=$ $3.3 \mathrm{~Hz}), 116.0$ (d, $J=21.9 \mathrm{~Hz}), 44.9$ (t, $J=7.2 \mathrm{~Hz})$. FT-IR $\left(v_{\max }, \mathrm{cm}^{-1}\right): 3447,2961$, $2150,1607,1510,1442,1418,1260,1229,1082,1017,800$.

These data are in good agreement with literature report. ${ }^{\text {S7 }}$<smiles>Cc1cccc(CN)c1</smiles>

1-(isocyanomethyl)-3-methylbenzene (3o; $44 \mathrm{mg}, 84 \%)$. Eluted with petroleum ether/ethyl acetate $=20: 1(\mathrm{v} / \mathrm{v})$. Yellow oil; ${ }^{1} \mathrm{H}$ NMR $\left(400 \mathrm{MHz}, \mathrm{CDCl}_{3}\right) \delta 7.29(\mathrm{t}, J=$ $7.9 \mathrm{~Hz}, 1 \mathrm{H}), 7.20-7.12(\mathrm{~m}, 3 \mathrm{H}), 4.60$ (s, 2H), 2.39 (s, 3H). ${ }^{13} \mathrm{C}$ NMR (101 MHz, $\left.\mathrm{CDCl}_{3}\right) \delta 157.5$ (t, $J=5.3 \mathrm{~Hz}$ ), 138.9 (s), 132.3 (s), 129.2 (s), 128.9 (s), 127.3 (s), $123.7(\mathrm{~s}), 45.5$ (t, $J=7.2 \mathrm{~Hz}), 21.4(\mathrm{~s})$. FT-IR $\left(v_{\max }, \mathrm{cm}^{-1}\right): 3439,2961,2149,1611$, 1491, 1440, 1342, 1260, 1094, 1039, 785,692. Anal. Calcd for $\mathrm{C}_{9} \mathrm{H}_{9} \mathrm{~N}: \mathrm{C}, 82.41 ; \mathrm{H}$, 6.92; N, 10.68. Found: C, 82.28; H, 6.84; N, 10.78 .

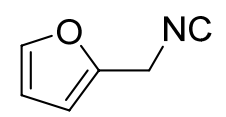

2-(isocyanomethyl)furan (3p; $17 \mathrm{mg}, 38 \%$ ). Eluted with petroleum ether/ethyl acetate $=20: 1(\mathrm{v} / \mathrm{v})$. Yellow oil; ${ }^{1} \mathrm{H}$ NMR $\left(400 \mathrm{MHz}, \mathrm{CDCl}_{3}\right) \delta 7.42(\mathrm{t}, J=1.2 \mathrm{~Hz}$, $1 \mathrm{H}), 6.38(\mathrm{~d}, J=1.3 \mathrm{~Hz}, 2 \mathrm{H}), 4.60(\mathrm{br} \mathrm{s}, 2 \mathrm{H}) .{ }^{13} \mathrm{C} \mathrm{NMR}\left(101 \mathrm{MHz}, \mathrm{CDCl}_{3}\right) \delta 157.9(\mathrm{t}$, $J=4.8 \mathrm{~Hz}), 145.5(\mathrm{~s}), 143.3(\mathrm{~s}), 110.7$ (s), 108.9 (s), 38.8 (t, $J=7.6 \mathrm{~Hz})$. FT-IR ( $v_{\max }$, $\mathrm{cm}^{-1}$ ): 3467, 3443, 2961, 2147, 1747, 1668, 1489, 1398, 1260, 1082, 1020, 799. (Low isolated yield due to significant loss of $3 \mathbf{p}$ with highly volatile property).

These data are in good agreement with literature report. ${ }^{\mathrm{S} 6}$ 
(1)

(S)-(1-isocyanoethyl)benzene (3q; $43 \mathrm{mg}, 81 \%$ ). Eluted with petroleum ether/ethyl acetate $=20: 1(\mathrm{v} / \mathrm{v})$. Colorless liquid; ${ }^{1} \mathrm{H}$ NMR $\left(400 \mathrm{MHz}, \mathrm{CDCl}_{3}\right) \delta 7.44-7.31(\mathrm{~m}$, $5 \mathrm{H}), 4.83(\mathrm{qt}, J=6.9,1.8 \mathrm{~Hz}, 1 \mathrm{H}), 1.69(\mathrm{dt}, J=6.9,2.2 \mathrm{~Hz}, 3 \mathrm{H}) .{ }^{13} \mathrm{C}$ NMR $(101 \mathrm{MHz}$, $\left.\mathrm{CDCl}_{3}\right) \delta 156.4(\mathrm{t}, J=4.8 \mathrm{~Hz}), 138.6(\mathrm{~s}), 129.0(\mathrm{~s}), 128.3(\mathrm{~s}), 125.4(\mathrm{~s}), 53.8$ (t, $J=$ $6.4 \mathrm{~Hz}), 25.1$ (s). FT-IR ( $\left.v_{\max }, \mathrm{cm}^{-1}\right): 3444,2988,2139,1496,1450,1377,1349,1260$, 1076, 1028, 799, 758, 697.

These data are in good agreement with literature report. ${ }^{\text {S8 }}$<smiles></smiles>

(2-isocyanoethyl)benzene (3r; $40 \mathrm{mg}, 76 \%$ ). Eluted with petroleum ether/ethyl acetate $=20: 1(\mathrm{v} / \mathrm{v})$. Yellow oil; ${ }^{1} \mathrm{H}$ NMR $\left(400 \mathrm{MHz}, \mathrm{CDCl}_{3}\right) \delta 7.44-7.18(\mathrm{~m}, 5 \mathrm{H})$, $3.61(\mathrm{tt}, J=7.1,1.9 \mathrm{~Hz}, 2 \mathrm{H}), 2.99(\mathrm{tt}, J=7.1,2.0 \mathrm{~Hz}, 2 \mathrm{H}) .{ }^{13} \mathrm{C}$ NMR (101 MHz, $\left.\mathrm{CDCl}_{3}\right) \delta 156.6(\mathrm{t}, J=5.4 \mathrm{~Hz}), 136.7(\mathrm{~s}), 128.8(\mathrm{~s}), 128.7(\mathrm{~s}), 127.3(\mathrm{~s}), 43.0(\mathrm{t}, J=$ $6.6 \mathrm{~Hz}), 35.7$ (s). FT-IR ( $\left.v_{\max }, \mathrm{cm}^{-1}\right): 3440,2962,2146,1495,1454,1352,1260,1082$, $1028,800,699$.

These data are in good agreement with literature report. ${ }^{\mathrm{S} 5}$<smiles></smiles>

1-(2-isocyanoethyl)-4-methoxybenzene (3s; $56 \mathrm{mg}, 86 \%$ ). Eluted with petroleum ether/ethyl acetate $=20: 1(\mathrm{v} / \mathrm{v})$. Colorless liquid; ${ }^{1} \mathrm{H}$ NMR $\left(400 \mathrm{MHz}, \mathrm{CDCl}_{3}\right) \delta 7.15$ $(\mathrm{d}, J=8.7 \mathrm{~Hz}, 2 \mathrm{H}), 6.88(\mathrm{~d}, J=8.7 \mathrm{~Hz}, 2 \mathrm{H}), 3.80(\mathrm{~s}, 3 \mathrm{H}), 3.56(\mathrm{tt}, J=7.1,1.8 \mathrm{~Hz}$, 2H), $2.92(\mathrm{tt}, J=7.0,1.8 \mathrm{~Hz}, 2 \mathrm{H}) .{ }^{13} \mathrm{C} \mathrm{NMR}\left(101 \mathrm{MHz}, \mathrm{CDCl}_{3}\right) \delta 158.8(\mathrm{~s}), 156.4(\mathrm{t}$, $J=5.6 \mathrm{~Hz}$ ), 129.8 (s), 128.8 (s), 114.2 (s), 55.3 (s), 43.3 (t, $J=6.5 \mathrm{~Hz}), 34.8$ (s). FT-IR $\left(v_{\max }, \mathrm{cm}^{-1}\right)$ : 2957, 2835, 2147, 1612, 1513, 1455, 1301, 1249, 1178, 1111, 
$1033,805$.

These data are in good agreement with literature report. ${ }^{\mathrm{S} 9}$<smiles>C=NC(C)CCc1ccccc1</smiles>

(3-isocyanobutyl)benzene (3t; $58 \mathrm{mg}, 90 \%$ ). Eluted with petroleum ether/ethyl acetate $=20: 1(\mathrm{v} / \mathrm{v})$. Yellow oil; ${ }^{1} \mathrm{H}$ NMR $\left(400 \mathrm{MHz} \mathrm{CDCl}_{3}\right) \delta 7.37-7.19(\mathrm{~m}, 5 \mathrm{H})$, $3.66-3.53(\mathrm{~m}, 1 \mathrm{H}), 2.94-2.70(\mathrm{~m}, 2 \mathrm{H}), 2.03-1.77(\mathrm{~m}, 2 \mathrm{H}), 1.40(\mathrm{dt}, J=6.6,2.2$ $\mathrm{Hz}, 3 \mathrm{H}) .{ }^{13} \mathrm{C} \mathrm{NMR}\left(101 \mathrm{MHz}, \mathrm{CDCl}_{3}\right) \delta 155.0$ (t, $\left.J=5.1 \mathrm{~Hz}\right), 140.3$ (s), 128.7 (s), $128.5(\mathrm{~s}), 126.4(\mathrm{~s}), 49.5(\mathrm{t}, J=5.6 \mathrm{~Hz}), 38.4(\mathrm{~s}), 32.0(\mathrm{~s}), 21.7(\mathrm{~s})$. FT-IR $\left(v_{\max }, \mathrm{cm}^{-1}\right)$ : 3027, 2938, 2137, 1603, 1495, 1454, 1347, 1109, 1052, 699.

These data are in good agreement with literature report. ${ }^{\text {S10 }}$<smiles>CC(C)(C)c1ccccc1</smiles>

(2-isocyanopropan-2-yl)benzene (3u; $45 \mathrm{mg}, 78 \%)$. Eluted with petroleum ether/ethyl acetate $=20: 1(\mathrm{v} / \mathrm{v})$. Yellow oil; ${ }^{1} \mathrm{H}$ NMR $\left(400 \mathrm{MHz}, \mathrm{CDCl}_{3}\right) \delta 7.51-7.46$ (m, 2H), $7.43-7.37(\mathrm{~m}, 2 \mathrm{H}), 7.35-7.29(\mathrm{~m}, 1 \mathrm{H}), 1.79(\mathrm{t}, J=2.0 \mathrm{~Hz}, 6 \mathrm{H})$.

${ }^{13} \mathrm{C}$ NMR (101 MHz, $\left.\mathrm{CDCl}_{3}\right) \delta 155.3$ (t, $\left.J=4.4 \mathrm{~Hz}\right), 142.3$ (s), 128.7 (s), 127.8 (s), $124.2(\mathrm{~s}), 60.7(\mathrm{t}, J=5.9 \mathrm{~Hz}), 32.0(\mathrm{~s})$. FT-IR $\left(v_{\max }, \mathrm{cm}^{-1}\right): 3446,2987,2133,1602$, 1447, 1368, 1257, 1166, 763, 698.

These data are in good agreement with literature report. ${ }^{\text {S11 }}$<smiles>N#CCc1ccc(N)cc1</smiles>

4-(2-isocyanoethyl)aniline (3v. $29 \mathrm{mg}, 50 \%$ ). Eluted with petroleum ether/ethyl acetate $=20: 1(\mathrm{v} / \mathrm{v})$. Yellow oil; ${ }^{1} \mathrm{H}$ NMR $\left(400 \mathrm{MHz}, \mathrm{CDCl}_{3}\right) \delta 7.01(\mathrm{~d}, J=8.3 \mathrm{~Hz}$, 2H), $6.65(\mathrm{~d}, J=8.3 \mathrm{~Hz}, 2 \mathrm{H}), 3.62-3.42(\mathrm{~m}, 4 \mathrm{H}), 2.87(\mathrm{t}, J=7.1 \mathrm{~Hz}, 2 \mathrm{H}) .{ }^{13} \mathrm{C} \mathrm{NMR}$ 
$\left(101 \mathrm{MHz}, \mathrm{CDCl}_{3}\right) \delta 154.9(\mathrm{t}), 144.5(\mathrm{~s}), 128.6(\mathrm{~s}), 125.5(\mathrm{~s}), 114.3(\mathrm{~s}), 42.4(\mathrm{t}), 33.9$ (s). FT-IR (vmax, $\mathrm{cm}^{-1}$ ): 2961, 2148, 1621, 1517, 1260, 1092, 1020, 800. Anal. Calcd for $\mathrm{C}_{9} \mathrm{H}_{10} \mathrm{~N}_{2}$ : C, 73.94; $\mathrm{H}, 6.89 ; \mathrm{N}, 19.16$. Found: C, 73.80; H, 6.76; N, 19.35.

Following the general procedure using anilines with an ortho-OH or ortho- $\mathrm{NH}_{2}$ group (4a-c), and an ethylenediamine compound 4d, benzoxazoles and benzimidazole 5a-c, and dihydroimidazole $\mathbf{5 d}$ were readily obtained.<smiles>Cc1ccc2ncoc2c1</smiles>

6-methylbenzo[d]oxazole (5a; $35 \mathrm{mg}, 65 \%$ ). Eluted with petroleum ether/ethyl acetate $=10: 1(\mathrm{v} / \mathrm{v})$. White solid; ${ }^{1} \mathrm{H}$ NMR $\left(400 \mathrm{MHz}, \mathrm{CDCl}_{3}\right) \delta 8.00(\mathrm{~s}, 1 \mathrm{H}), 7.63(\mathrm{~d}$, $J=8.1 \mathrm{~Hz}, 1 \mathrm{H}), 7.35(\mathrm{~s}, 1 \mathrm{H}), 7.15(\mathrm{~d}, J=8.1 \mathrm{~Hz}, 1 \mathrm{H}), 2.46(\mathrm{~s}, 3 \mathrm{H}) .{ }^{13} \mathrm{C}$ NMR $(101$ $\left.\mathrm{MHz}, \mathrm{CDCl}_{3}\right) \delta 152.0(\mathrm{~s}), 150.3$ (s), 137.8 (s), 136.0 (s), 125.8 (s), 119.9 (s), 111.0 (s), $21.7(\mathrm{~s})$.

These data are in good agreement with literature report. ${ }^{\mathrm{S} 12}$<smiles>c1ccc2ocnc2c1</smiles>

benzo[d]oxazole $(5 \mathbf{b} ; 17 \mathrm{mg}, 35 \%)$. Eluted with petroleum ether/ethyl acetate $=10: 1$ (v/v). Yellow oil; ${ }^{1} \mathrm{H}$ NMR (400 MHz, $\left.\mathrm{CDCl}_{3}\right) \delta 8.09(\mathrm{~s}, 1 \mathrm{H}), 7.83-7.76(\mathrm{~m}, 1 \mathrm{H})$, $7.61-7.55(\mathrm{~m}, 1 \mathrm{H}), 7.42-7.33(\mathrm{~m}, 2 \mathrm{H}) .{ }^{13} \mathrm{C} \mathrm{NMR}\left(101 \mathrm{MHz}, \mathrm{CDCl}_{3}\right) \delta 152.5(\mathrm{~s})$, $150.0(\mathrm{~s}), 140.0(\mathrm{~s}), 125.6(\mathrm{~s}), 124.6(\mathrm{~s}), 120.6(\mathrm{~s}), 111.0(\mathrm{~s})$.

These data are in good agreement with literature report. ${ }^{\mathrm{S} 13}$<smiles>FC(F)(F)n1cnc2ccccc21</smiles>

1-(difluoromethyl)-1H-benzo[d]imidazole (5c; $34 \mathrm{mg}, 50 \%)$. Eluted with petroleum ether/ethyl acetate $=10: 1(\mathrm{v} / \mathrm{v})$. Yellow oil; ${ }^{1} \mathrm{H}$ NMR $\left(400 \mathrm{MHz}, \mathrm{CDCl}_{3}\right) \delta 8.12(\mathrm{~s}$, 1H), $7.89-7.81(\mathrm{~m}, 1 \mathrm{H}), 7.65-7.57(\mathrm{~m}, 1 \mathrm{H}), 7.44-7.35(\mathrm{~m}, 2 \mathrm{H}), 7.33(\mathrm{t}, J=60.4$ 
$\mathrm{Hz}, 1 \mathrm{H}) .{ }^{13} \mathrm{C}$ NMR $\left(101 \mathrm{MHz}, \mathrm{CDCl}_{3}\right) \delta 143.9$ (s), 139.1 (s), 130.5 (s), 124.8 (s), 124.2 (s), 121.0 (s), 111.1 (s), 109.0 (t, $J=249.9 \mathrm{~Hz}) .{ }^{19} \mathrm{~F}$ NMR $\left(376 \mathrm{MHz}, \mathrm{CDCl}_{3}\right) \delta$ $-93.7(\mathrm{~s})$.

These data are in good agreement with literature report. ${ }^{\text {S14 }}$<smiles>Cc1ccc(S(=O)(=O)N2C=NC(c3ccccc3)C2c2ccccc2)cc1</smiles>

4,5-diphenyl-1-tosyl-4,5-dihydro-1H-imidazole (5d; $107 \mathrm{mg}, 72 \%$ ). Eluted with petroleum ether/ethyl acetate $=4: 1(\mathrm{v} / \mathrm{v})$. Colorless oil; ${ }^{1} \mathrm{H}$ NMR $\left(400 \mathrm{MHz}, \mathrm{CDCl}_{3}\right) \delta$ $7.81(\mathrm{~d}, J=2.0 \mathrm{~Hz}, 1 \mathrm{H}), 7.54(\mathrm{~d}, J=8.3 \mathrm{~Hz}, 2 \mathrm{H}), 7.33-7.19(\mathrm{~m}, 8 \mathrm{H}), 7.19-7.12(\mathrm{~m}$, 2H), $6.84(\mathrm{dd}, J=7.7,1.6 \mathrm{~Hz}, 2 \mathrm{H}), 5.08$ (dd, $J=7.4,2.0 \mathrm{~Hz}, 1 \mathrm{H}), 4.55$ (d, $J=7.4 \mathrm{~Hz}$, 1H), 2.43 (s, 3H). ${ }^{13} \mathrm{C}$ NMR (101 MHz, $\left.\mathrm{CDCl}_{3}\right) \delta 148.5$ (s), 144.7 (s), 140.8 (s), 139.2 (s), 134.4 (s), 129.9 (s), 128.9 (s), 128.7 (s), 128.3 (s), 127.9 (s), 127.4 (s), 126.9 (s), 126.3 (s), 82.3 (s), 69.9 (s), 21.6 (s). HRMS (ESI) m/z calcd for $\mathrm{C}_{22} \mathrm{H}_{21} \mathrm{~N}_{2} \mathrm{O}_{2} \mathrm{~S}^{+}$ $(\mathrm{M}+\mathrm{H})^{+}$377.1324, found 377.1318 .

\section{Late-stage functionalization of biologically active amines}<smiles>CCOC(=O)C1=C(COCCN=[N+]=[N-])NC(C)=C(C(=O)OC)C1c1ccccc1Cl</smiles>

3-ethyl

5-methyl 4-(2-chlorophenyl)-2-((2-isocyanoethoxy)methyl)-6-methyl-1,4-dihydropyridine-3 ,5-dicarboxylate (3x; $125 \mathrm{mg}, 75 \%)$. Eluted with petroleum ether/ethyl acetate $=4: 1$ (v/v). White solid; ${ }^{1} \mathrm{H}$ NMR (400 MHz, $\left.\mathrm{CDCl}_{3}\right) \delta 7.37$ (dd, $\left.J=7.7,1.3 \mathrm{~Hz}, 1 \mathrm{H}\right), 7.22$ $(\mathrm{d}, J=7.1 \mathrm{~Hz}, 1 \mathrm{H}), 7.13(\mathrm{t}, J=7.5 \mathrm{~Hz}, 1 \mathrm{H}), 7.03(\mathrm{t}, J=6.8 \mathrm{~Hz}, 2 \mathrm{H}), 5.40(\mathrm{~s}, 1 \mathrm{H})$, $4.78(\mathrm{q}, J=15.9 \mathrm{~Hz}, 2 \mathrm{H}), 4.03(\mathrm{dtt}, J=10.8,7.2,3.7 \mathrm{~Hz}, 2 \mathrm{H}), 3.73(\mathrm{~d}, J=4.1 \mathrm{~Hz}$, $2 \mathrm{H}), 3.64(\mathrm{t}, J=4.9 \mathrm{~Hz}, 2 \mathrm{H}), 3.59(\mathrm{~s}, 3 \mathrm{H}), 2.35(\mathrm{~s}, 3 \mathrm{H}), 1.17(\mathrm{t}, J=7.1 \mathrm{~Hz}, 3 \mathrm{H}) .{ }^{13} \mathrm{C}$ 
NMR (101 MHz, $\left.\mathrm{CDCl}_{3}\right) \delta 167.9$ (s), 167.1 (s), 158.3 (t, $\left.J=4.5 \mathrm{~Hz}\right), 145.6(\mathrm{~s}), 144.3$ (s), 144.0 (s), 132.4 (s), 131.5 (s), 129.3 (s), 127.5 (s), 126.9 (s), 104.0 (s), 102.0 (s), 68.3 (s), 67.9 (s), 59.9 (s), 50.8 (s), 41.7 (t, $J=6.4 \mathrm{~Hz}$ ), 37.2 (s), 19.6 (s), 14.2 (s). HRMS (ESI) m/z calcd for $\mathrm{C}_{21} \mathrm{H}_{23} \mathrm{ClN}_{2} \mathrm{O}_{5} \mathrm{Na}^{+}(\mathrm{M}+\mathrm{Na})^{+} 441.1193$, found 441.1187.<smiles>CNCc1ccc(N2CCOCC2)cc1</smiles>

4-(4-(isocyanomethyl)phenyl)morpholine (3y; $52 \mathrm{mg}, 67 \%$ ). Eluted with petroleum ether/ethyl acetate $=20: 1(\mathrm{v} / \mathrm{v})$. Colorless oil; ${ }^{1} \mathrm{H}$ NMR $\left(400 \mathrm{MHz}, \mathrm{CDCl}_{3}\right) \delta 7.24(\mathrm{~d}$, $J=8.7 \mathrm{~Hz}, 2 \mathrm{H}), 6.91(\mathrm{~d}, J=8.7 \mathrm{~Hz}, 2 \mathrm{H}), 4.54(\mathrm{~s}, 2 \mathrm{H}), 3.90-3.81(\mathrm{~m}, 4 \mathrm{H}), 3.23-$ $3.08(\mathrm{~m}, 4 \mathrm{H}) .{ }^{13} \mathrm{C}$ NMR (101 MHz, $\left.\mathrm{CDCl}_{3}\right) \delta 156.9(\mathrm{t}), 151.3(\mathrm{~s}), 127.9(\mathrm{~s}), 123.5(\mathrm{~s})$, $115.7(\mathrm{~s}), 66.8(\mathrm{~s}), 49.0(\mathrm{~s}), 45.1(\mathrm{t})$.<smiles>C=NC[C@H]1CCC[C@]2(C)c3ccc(C(C)C)cc3CC[C@H]12</smiles>

$(1 R, 4 a S, 10 a R)-1-(i s o c y a n o m e t h y l)-7-i s o p r o p y l-1,4 a-d i m e t h y l-1,2,3,4,4 a, 9,10,10 a-$ octahydrophenanthrene (3z; $91 \mathrm{mg}, 77 \%)$. Eluted with petroleum ether/ethyl acetate $=10: 1(\mathrm{v} / \mathrm{v})$. Colorless oil; ${ }^{1} \mathrm{H}$ NMR $\left(400 \mathrm{MHz}, \mathrm{CDCl}_{3}\right) \delta 7.21(\mathrm{~d}, J=8.2 \mathrm{~Hz}, 1 \mathrm{H})$, $7.04(\mathrm{dd}, J=8.2,1.7 \mathrm{~Hz}, 1 \mathrm{H}), 6.93(\mathrm{~s}, 1 \mathrm{H}), 3.35$ (d, $J=14.7 \mathrm{~Hz}, 1 \mathrm{H}), 3.19$ (d, $J=$ $14.7 \mathrm{~Hz}, 1 \mathrm{H}), 2.95(\mathrm{dd}, J=8.3,4.5 \mathrm{~Hz}, 2 \mathrm{H}), 2.91-2.81(\mathrm{~m}, 1 \mathrm{H}), 2.34(\mathrm{dt}, J=12.8$, $2.7 \mathrm{~Hz}, 1 \mathrm{H}), 1.79(\mathrm{ddd}, J=12.5,10.8,7.0 \mathrm{~Hz}, 4 \mathrm{H}), 1.73-1.66(\mathrm{~m}, 2 \mathrm{H}), 1.56(\mathrm{t}, J=$ $3.8 \mathrm{~Hz}, 2 \mathrm{H}), 1.27$ (s, 3H), $1.26(\mathrm{~s}, 6 \mathrm{H}), 1.02(\mathrm{~s}, 3 \mathrm{H}) .{ }^{13} \mathrm{C} \mathrm{NMR}\left(101 \mathrm{MHz}, \mathrm{CDCl}_{3}\right) \delta$ $157.1(\mathrm{t}), 146.6(\mathrm{~s}), 145.8(\mathrm{~s}), 134.4(\mathrm{~s}), 126.9(\mathrm{~s}), 124.4(\mathrm{~s}), 124.1(\mathrm{~s}), 53.7(\mathrm{t}), 45.1$ (s), 38.1 (s), 37.5 (s), 37.0 (s), 36.0 (s), 33.5 (s), 30.1 (s), 25.1 (s), 24.0 (d, J=1.1 Hz), 19.0 (s), 18.6 (s), 18.1 (s). HRMS (ESI) m/z calcd for $\mathrm{C}_{21} \mathrm{H}_{29} \mathrm{NNa}^{+}(\mathrm{M}+\mathrm{Na})^{+}$318.2198, found 318.2193. 


\section{References:}

S1. Leifert, D.; Artiukhin, D. G.; Neugebauer, J.; Galstyan, A.; Strassert, C. A.; Studer, A. Chem. Commun. 2016, 52, 5997.

S2. Michalik, D.; Schaks, A.; Wessjohann, L. A. Eur. J. Org. Chem. 2007, 149.

S3. Baena, M. J.; Coco, S.; Espinet, P. Cryst. Growth Des. 2015, 15, 1611.

S4. Neo, A. G.; Marcos, C. F.; Marcaccini, S.; Pepino, R. Tetrahedron Lett. 2005, 46, 7977.

S5. Guirado, A.; Zapata, A.; Gomez, J. L.; Trabalon, L. Galvez, J. Tetrahedron 1999, $55,9631$.

S6. Porcheddu, A.; Giacomelli, G.; Salaris, M. J. Org. Chem. 2005, 70, 2361.

S7. Mocci, R.; Murgia, S.; De Luca, L.; Colacino, E.; Delogu, F.; Porcheddu, A. Org. Chem. Front. 2018, 5, 531.

S8. Flesch, D. et al. J. Med. Chem. 2017, 60, 7199.

S9. Mampuys, P.; Neumann, H.; Sergeyev, S.; Orru, R. V. A.; Jiao, H.; Spannenberg, A.; Maes, B. U. W.; Beller, M. ACS Catal. 2017, 7, 5549.

S10. Adachi, M.; Miyasaka, T.; Hashimoto, H.; Nishikawa, T. Org. Lett. 2017, 19, 380.

S11. Alwedi, E.; Lujan-Montelongo, J. A.; Cortes-Mejia, R.; del Campo, J. M.; Altundas, B.; Fleming, F. F. Eur. J. Org. Chem. 2019, 4644.

S12. Guo, S.; Qian, B.; Xie, Y.; Xia, C.; Huang, H. Angew. Chem., Int. Ed. 2010, 49, 8417.

S13. Shirini, F.; Mamaghani, M.; Seddighi, M. Res. Chem Intermed. 2015, 41, 5611.

S14. Ma, X.; Mai, S.; Zhou, Y.; Cheng, G.-J.; Song, Q. Chem. Commun. 2018, 54, 8960. 


\section{General procedures for the Ugi reactions}

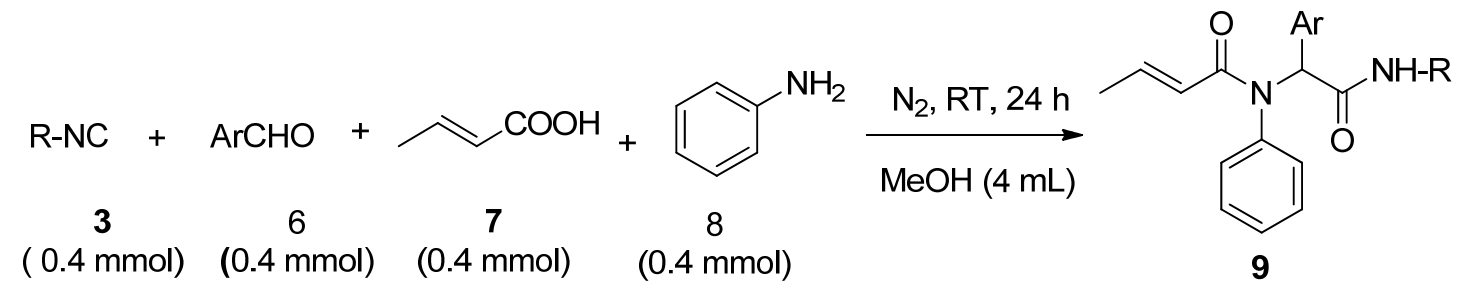

A solution of crotonic acid $7(0.4 \mathrm{mmol})$, aldehyde $6(0.4 \mathrm{mmol})$ and aniline 8 $(0.4 \mathrm{mmol})$ in $\mathrm{MeOH}(4 \mathrm{~mL})$ was stirred for $0.5 \mathrm{~h}$ at room temperature. Then isocyanide $3(0.4 \mathrm{mmol})$ was added to the reaction mixture which was further stirred vigorously. The reaction progress was monitored by TLC. After $24 \mathrm{~h}$, the solvent was removed under reduced pressure. The precipitates were purified by column chromatography using a mixture of petroleum ether (PE) and ethyl acetate (EA) as eluent to give amide products 9 .<smiles>C/C=C/C(=O)N(c1ccccc1)C(C(=O)NCc1ccccc1)c1ccc(Cl)cc1</smiles>

\section{(E)-N-(2-(benzylamino)-1-(4-chlorophenyl)-2-oxoethyl)- $N$-phenylbut-2-enamide}

(9a; $102 \mathrm{mg}, 61 \%)$. Eluted with petroleum ether/ethyl acetate $=2: 1(\mathrm{v} / \mathrm{v})$. White solid; ${ }^{1} \mathrm{H}$ NMR $\left(400 \mathrm{MHz}, \mathrm{CDCl}_{3}\right) \delta 7.39-7.08(\mathrm{~m}, 14 \mathrm{H}), 6.93(\mathrm{~m}, 1 \mathrm{H}), 6.55(\mathrm{t}, J=5.0 \mathrm{~Hz}$, 1H), $6.10(\mathrm{~s}, 1 \mathrm{H}), 5.60(\mathrm{dq}, J=15.1,1.6 \mathrm{~Hz}, 1 \mathrm{H}), 4.49$ (d, $J=5.8 \mathrm{~Hz}, 2 \mathrm{H}), 1.70(\mathrm{dd}, J$ $=6.9,1.4 \mathrm{~Hz}, 3 \mathrm{H}) .{ }^{13} \mathrm{C} \mathrm{NMR}\left(101 \mathrm{MHz}, \mathrm{CDCl}_{3}\right) \delta 169.5$ (s), 166.7 (s), 142.9 (s), 139.5 (s), 138.1 (s), 134.4 (s), 133.0 (s), 131.7 (s), 130.5 (s), 129.0 (s), 128.6 (s), 128.5 (s), 128.3 (s), 127.7 (s), 127.4 (s), 122.6 (s), 64.7 (s), 43.8 (s), 18.1 (s). 
<smiles>C/C=C/C(=O)N(c1ccccc1)C(C(=O)NC(C)(C)c1ccccc1)c1ccccc1</smiles>

(E)- $N$-(2-oxo-1-phenyl-2-((2-phenylpropan-2-yl)amino)ethyl)- $N$-phenylbut-2-ena mide (9b; $92 \mathrm{mg}, 56 \%)$. Eluted with petroleum ether/ethyl acetate $=2: 1(\mathrm{v} / \mathrm{v})$. White solid; ${ }^{1} \mathrm{H}$ NMR (400 MHz, $\left.\mathrm{CDCl}_{3}\right) \delta 7.44-7.10(\mathrm{~m}, 15 \mathrm{H}), 7.06-6.90(\mathrm{~m}, 1 \mathrm{H}), 6.54$ $(\mathrm{s}, 1 \mathrm{H}), 6.14(\mathrm{~s}, 1 \mathrm{H}), 5.63(\mathrm{dq}, J=15.1,1.2 \mathrm{~Hz}, 1 \mathrm{H}), 1.72(\mathrm{~m}, 9 \mathrm{H}) .{ }^{13} \mathrm{C}$ NMR $(101$ $\left.\mathrm{MHz}, \mathrm{CDCl}_{3}\right) \delta 168.7$ (s), 166.5 (s), 146.9 (s), 142.1 (s), 139.7 (s), 134.6 (s), 130.8 (s), 130.4 (s), 128.7 (s), 128.29, 128.25, $128.20,127.8$ (s), 126.5 (s), 124.9 (s), 123.1 (s), $65.6(\mathrm{~s}), 56.1(\mathrm{~s}), 29.2(\mathrm{~s}), 29.1(\mathrm{~s}), 18.1(\mathrm{~s})$.<smiles>C/C=C/C(=O)N(c1ccccc1)C(C(=O)NC(C)(C)c1ccccc1)c1ccc(OC)cc1</smiles>

(E)- $N$-(1-(4-methoxyphenyl)-2-oxo-2-((2-phenylpropan-2-yl)amino)ethyl)- $N$-phen ylbut-2-enamide (9c; $91 \mathrm{mg}, 52 \%$ ). Eluted with petroleum ether/ethyl acetate $=2: 1$ (v/v). White solid; ${ }^{1} \mathrm{H}$ NMR (400 MHz, $\left.\mathrm{CDCl}_{3}\right) \delta 7.40-6.88(\mathrm{~m}, 13 \mathrm{H}), 6.68(\mathrm{~d}, J=$ $8.8 \mathrm{~Hz}, 2 \mathrm{H}), 6.48(\mathrm{~s}, 1 \mathrm{H}), 6.11(\mathrm{~s}, 1 \mathrm{H}), 5.59(\mathrm{dq}, J=15.1,1.7 \mathrm{~Hz}, 1 \mathrm{H}), 3.72(\mathrm{~s}, 3 \mathrm{H})$, 1.73 - $1.64(\mathrm{~m}, 9 \mathrm{H}) .{ }^{13} \mathrm{C}$ NMR (101 MHz, $\left.\mathrm{CDCl}_{3}\right) \delta 168.9$ (s), 166.5 (s), 159.4 (s), 146.9 (s), 141.9 (s), 139.5 (s), 131.8 (s), 130.9 (s), 128.6 (s), 128.3 (s), 127.8 (s), 126.6 (s), 126.5 (s), 124.9 (s), 123.1 (s), 113.5 (s), 64.5 (s), 56.0 (s), 55.2 (s), 29.2 (d, $J=1.5 \mathrm{~Hz}), 18.0(\mathrm{~s})$. 

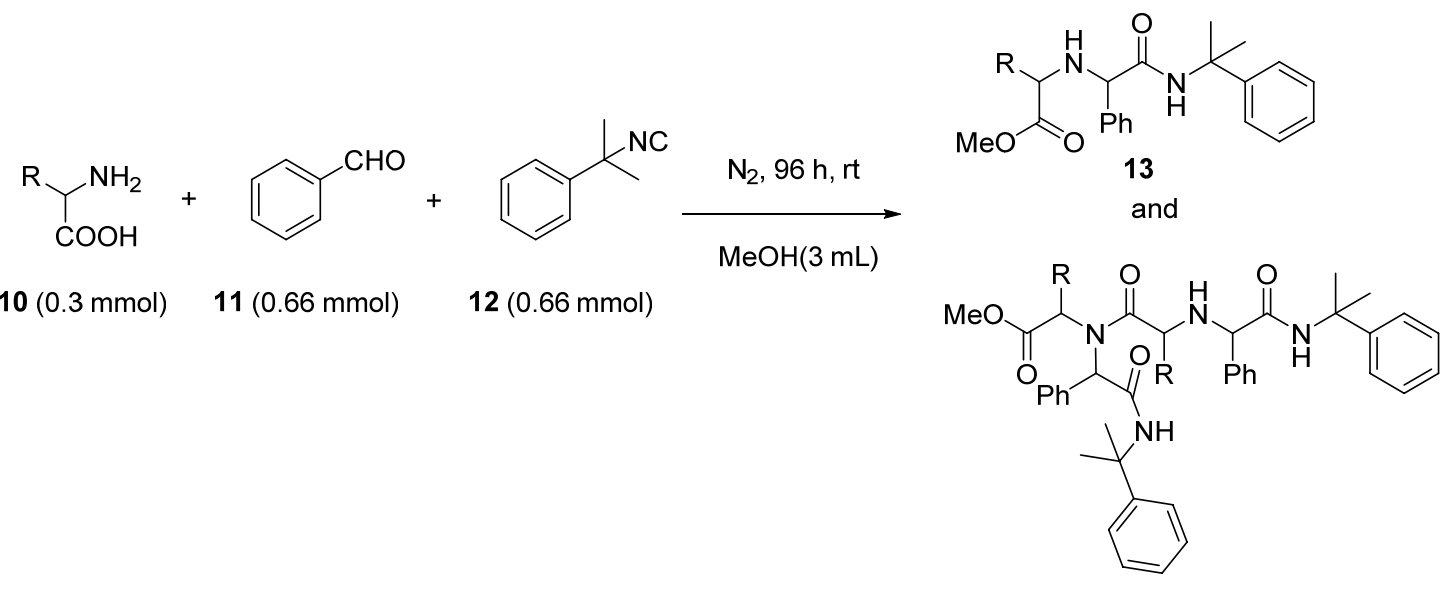

14

A solution of amino acid $10(0.3 \mathrm{mmol})$ and aldehyde $11(0.66 \mathrm{mmol})$ in $\mathrm{MeOH}$ $(3 \mathrm{~mL})$ was stirred for $0.5 \mathrm{~h}$ at room temperature. Then isocyanide $12(0.66 \mathrm{mmol})$ was added to the reaction mixture at the same temperature. The resulting mixture was stirred at $25{ }^{\circ} \mathrm{C}$ for $96 \mathrm{~h}$. The volatile was removed in vacuum, and the residue was dissolved in $1 \mathrm{~mL} \mathrm{CH}_{2} \mathrm{Cl}_{2}$. After precipitating in petroleum ether for three times and dried in vacuum at room temperature to afford polypeptoids $\mathbf{1 3}$ and $\mathbf{1 4}$ as white or light yellow solid. Product $\mathbf{1 4}$ results from the bimolecular condensation of $\mathbf{1 3}$ with the acylation of the secondary amine group in $\mathbf{1 3}$ by the methyl ester group in a second molecule of $\mathbf{1 3 .}$

Using $L$-phenylalanine as a reactant, after the reaction a total of $95 \mathrm{mg}$ of products. Using tryptophan as a reactant, after the reaction a total of $120 \mathrm{mg}$ of products. The monomer ester and dimer ester were determined as the main products.<smiles>COC(=O)C(Cc1ccccc1)NC(C(=O)NC(C)(C)c1ccccc1)c1ccccc1</smiles>

methyl (2-oxo-1-phenyl-2-((2-phenylpropan-2-yl)amino)ethyl)phenylalaninate (13a)

White solid; HRMS (ESI) $\mathrm{m} / \mathrm{z}$ calcd for $\mathrm{C}_{27} \mathrm{H}_{31} \mathrm{~N}_{2} \mathrm{O}_{3}{ }^{+}(\mathrm{M}+\mathrm{H})^{+} 431.2329$, found 431.2336. 
<smiles>COC(=O)C(Cc1ccccc1)N(C(=O)C(Cc1ccccc1)NC(C(=O)NC(C)(C)c1ccccc1)c1ccccc1)C(C(=O)NC(C)(C)c1ccccc1)c1ccccc1</smiles>

methyl

$N$-(2-oxo-1-phenyl-2-((2-phenylpropan-2-yl)amino)ethyl)- $N$-((2-oxo-1-phenyl-2-(( 2-phenylpropan-2-yl)amino)ethyl)phenylalanyl)phenylalaninate (14a)

White solid; HRMS (ESI) $\mathrm{m} / \mathrm{z}$ calcd for $\mathrm{C}_{53} \mathrm{H}_{57} \mathrm{~N}_{4} \mathrm{O}_{5}{ }^{+}(\mathrm{M}+\mathrm{H})^{+}$829.4323, found 829.4327.<smiles>COC(=O)C(Cc1c[nH]c2ccccc12)NC(C(=O)NC(C)(C)c1ccccc1)c1ccccc1</smiles>

methyl (2-oxo-1-phenyl-2-((2-phenylpropan-2-yl)amino)ethyl)tryptophanate (13b)

Yellow solid; HRMS (ESI) $\mathrm{m} / \mathrm{z}$ calcd for $\mathrm{C}_{29} \mathrm{H}_{32} \mathrm{~N}_{3} \mathrm{O}_{3}{ }^{+}(\mathrm{M}+\mathrm{H})^{+} 470.2438$, found 470.2442 .<smiles>COC(=O)C(Cc1c[nH]c2ccccc12)N(C(=O)C(Cc1c[nH]c2ccccc12)NC(C(=O)NC(C)(C)c1ccccc1)c1ccccc1)C(C(=O)NC(C)(C)c1ccccc1)c1ccccc1</smiles>

methyl

$N$-(2-oxo-1-phenyl-2-((2-phenylpropan-2-yl)amino)ethyl)- $N$-((2-oxo-1-phenyl-2-(( 2-phenylpropan-2-yl)amino)ethyl)tryptophyl)tryptophanate (14b) 
Yellow solid; HRMS (ESI) $\mathrm{m} / \mathrm{z}$ calcd for $\mathrm{C}_{57} \mathrm{H}_{59} \mathrm{~N}_{6} \mathrm{O}_{5}^{+}(\mathrm{M}+\mathrm{H})^{+}$907.4542, found 907.4541 . 
4. ${ }^{1} \mathrm{H},{ }^{19} \mathrm{~F}$ and ${ }^{13} \mathrm{C}$ NMR spectra for all the products

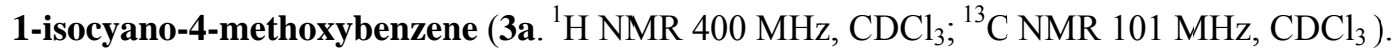
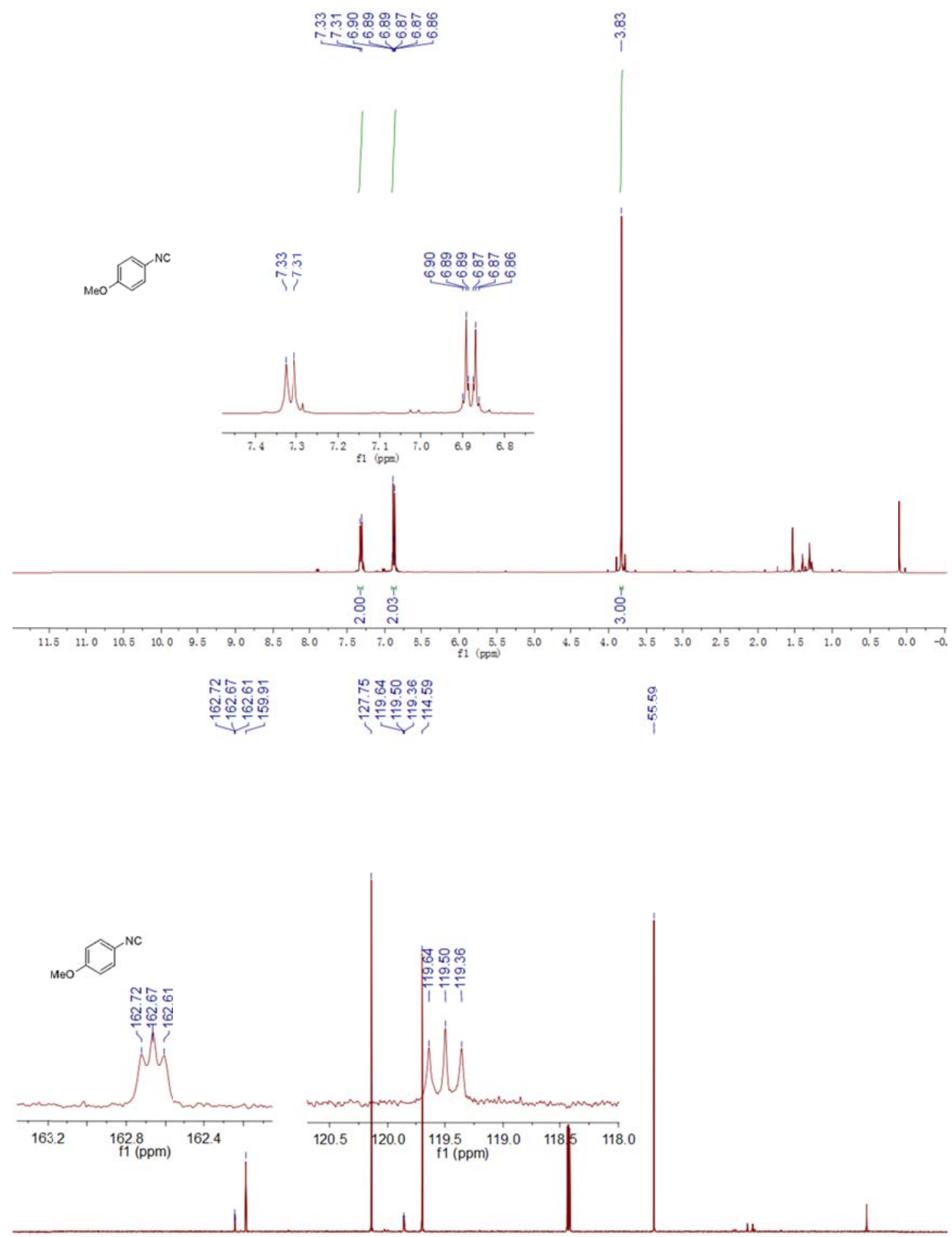

$\begin{array}{lllllllllllllllllllllll}210 & 200 & 190 & 180 & 170 & 160 & 150 & 140 & 130 & 120 & 110 \underset{\mathrm{f} 1(\mathrm{ppm})}{100} & 90 & 80 & 70 & 60 & 50 & 40 & 30 & 20 & 10 & 0 & -10\end{array}$ 
4-isocyano-N,N-dimethylaniline(3b. ${ }^{1} \mathrm{H}$ NMR $400 \mathrm{MHz}, \mathrm{CDCl}_{3} ;{ }^{13} \mathrm{C} \mathrm{NMR} 101 \mathrm{MHz}, \mathrm{CDCl}_{3}$ ).

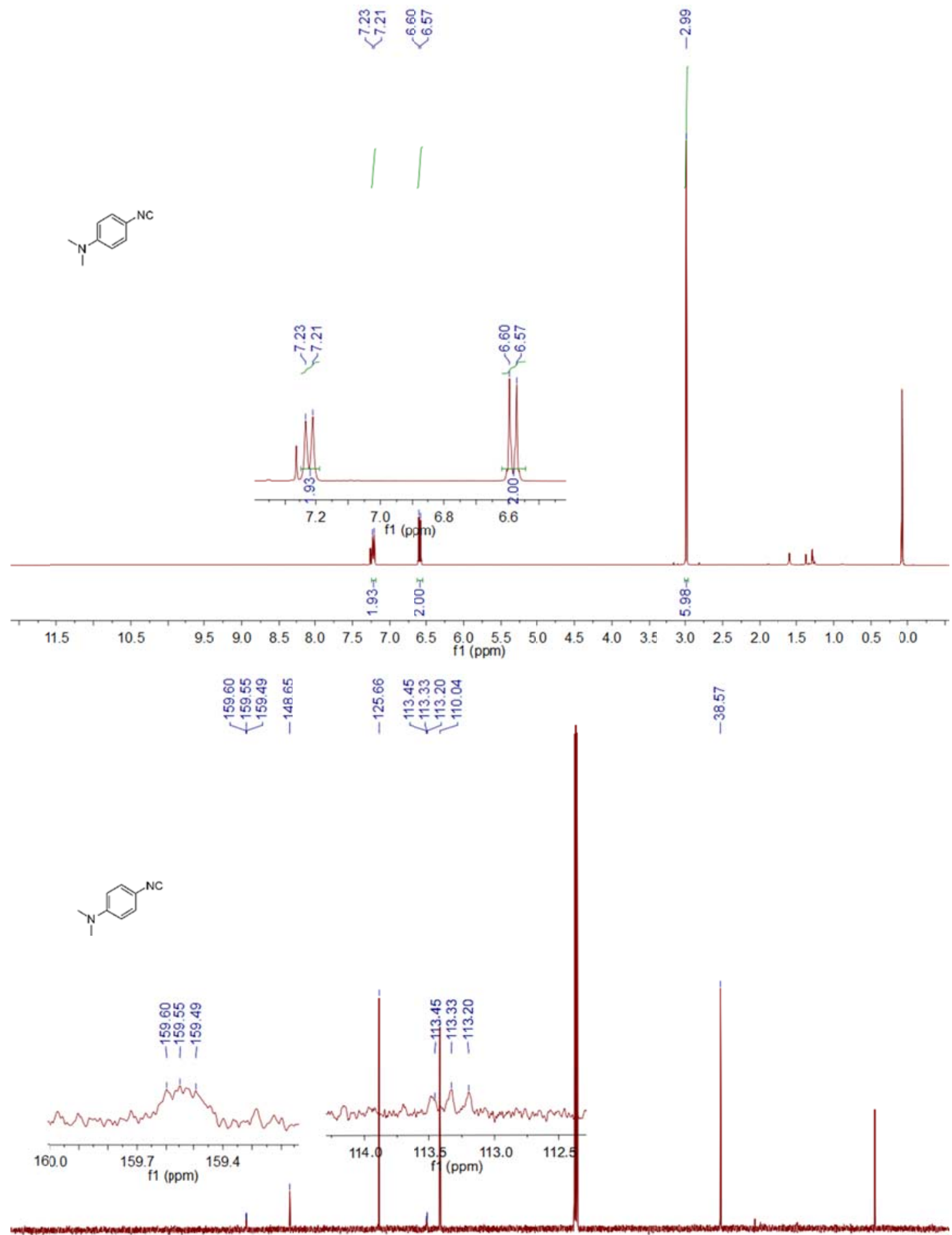

$\begin{array}{lllllllllllllllllllllll}210 & 200 & 190 & 180 & 170 & 160 & 150 & 140 & 130 & 120 & 110 & 100 & 90 & 80 & 70 & 60 & 50 & 40 & 30 & 20 & 10 & 0 & -10\end{array}$ 
4-isocyanophenol (3c. ${ }^{1} \mathrm{H}$ NMR $400 \mathrm{MHz}, \mathrm{CDCl}_{3} ;{ }^{13} \mathrm{C} \mathrm{NMR} 101 \mathrm{MHz}, \mathrm{CDCl}_{3}$ ).

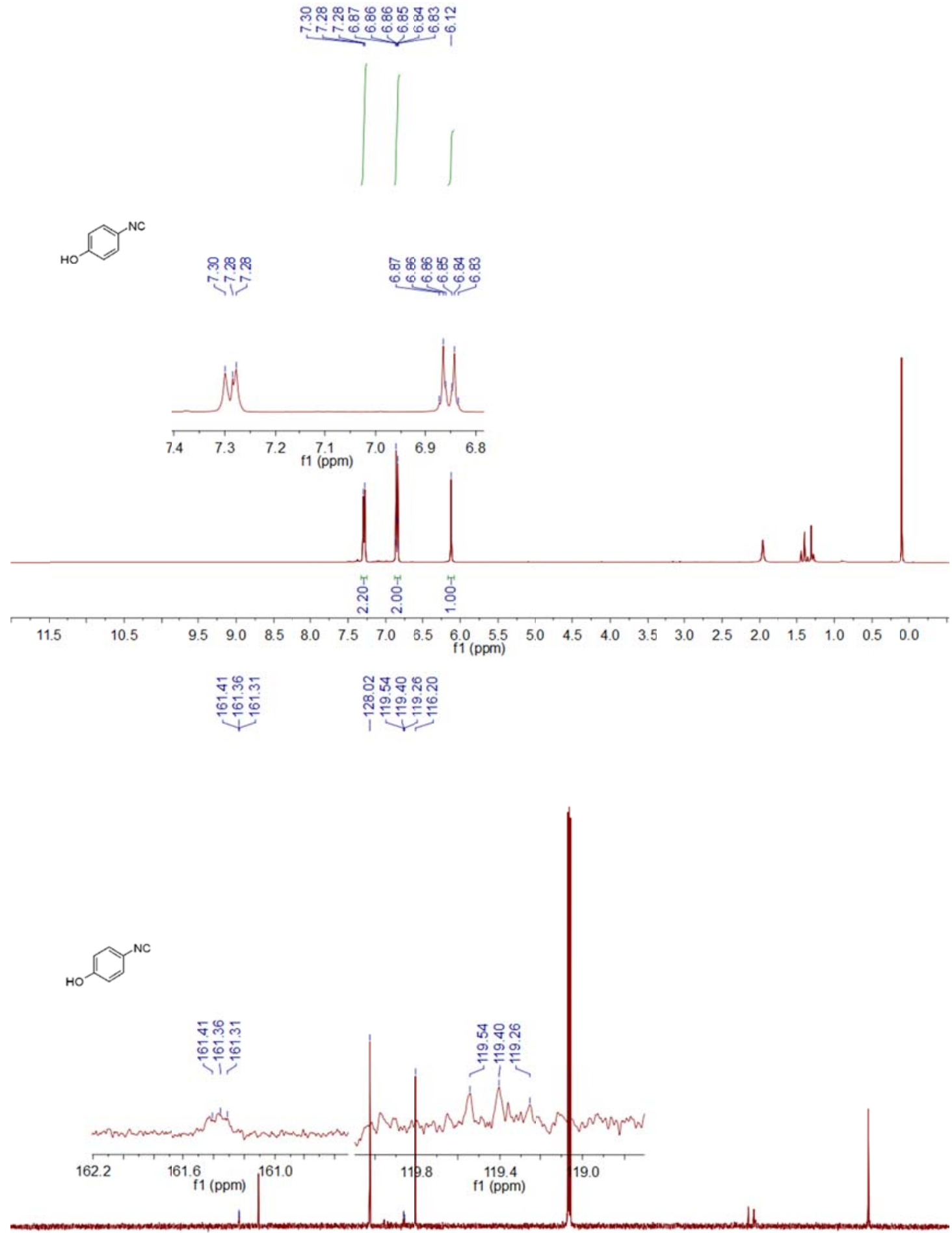

$\begin{array}{lllllllllllllllllllllll}210 & 200 & 190 & 180 & 170 & 160 & 150 & 140 & 130 & 120 & 110 & \begin{array}{l}100 \\ \mathrm{f} 1(\mathrm{ppm})\end{array} & 90 & 80 & 70 & 60 & 50 & 40 & 30 & 20 & 10 & 0 & -10\end{array}$ 


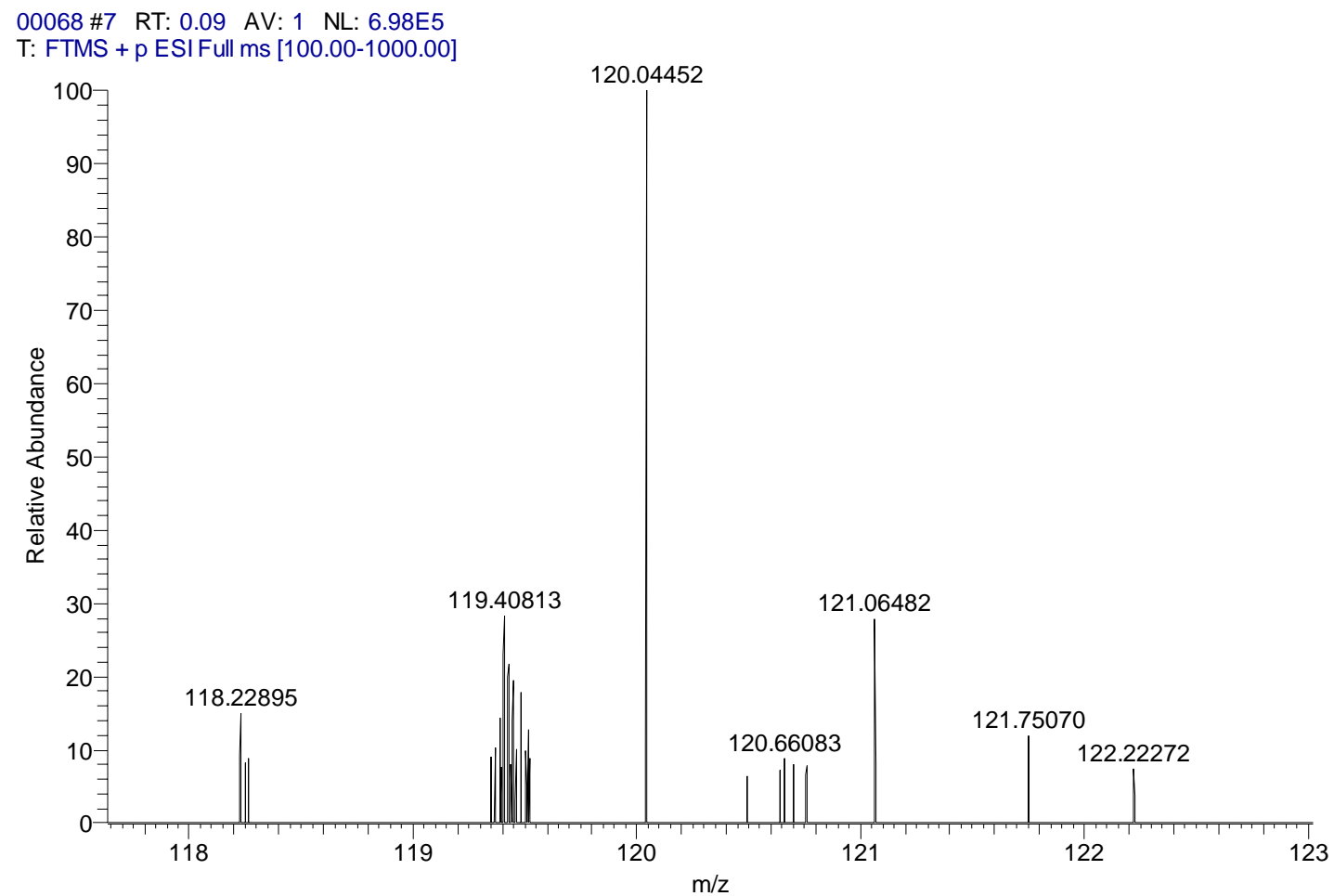


4-isocyanoaniline (3d. ${ }^{1} \mathrm{H}$ NMR $400 \mathrm{MHz}, \mathrm{CDCl}_{3} ;{ }^{13} \mathrm{C} \mathrm{NMR} 101 \mathrm{MHz}, \mathrm{CDCl}_{3}$ ).

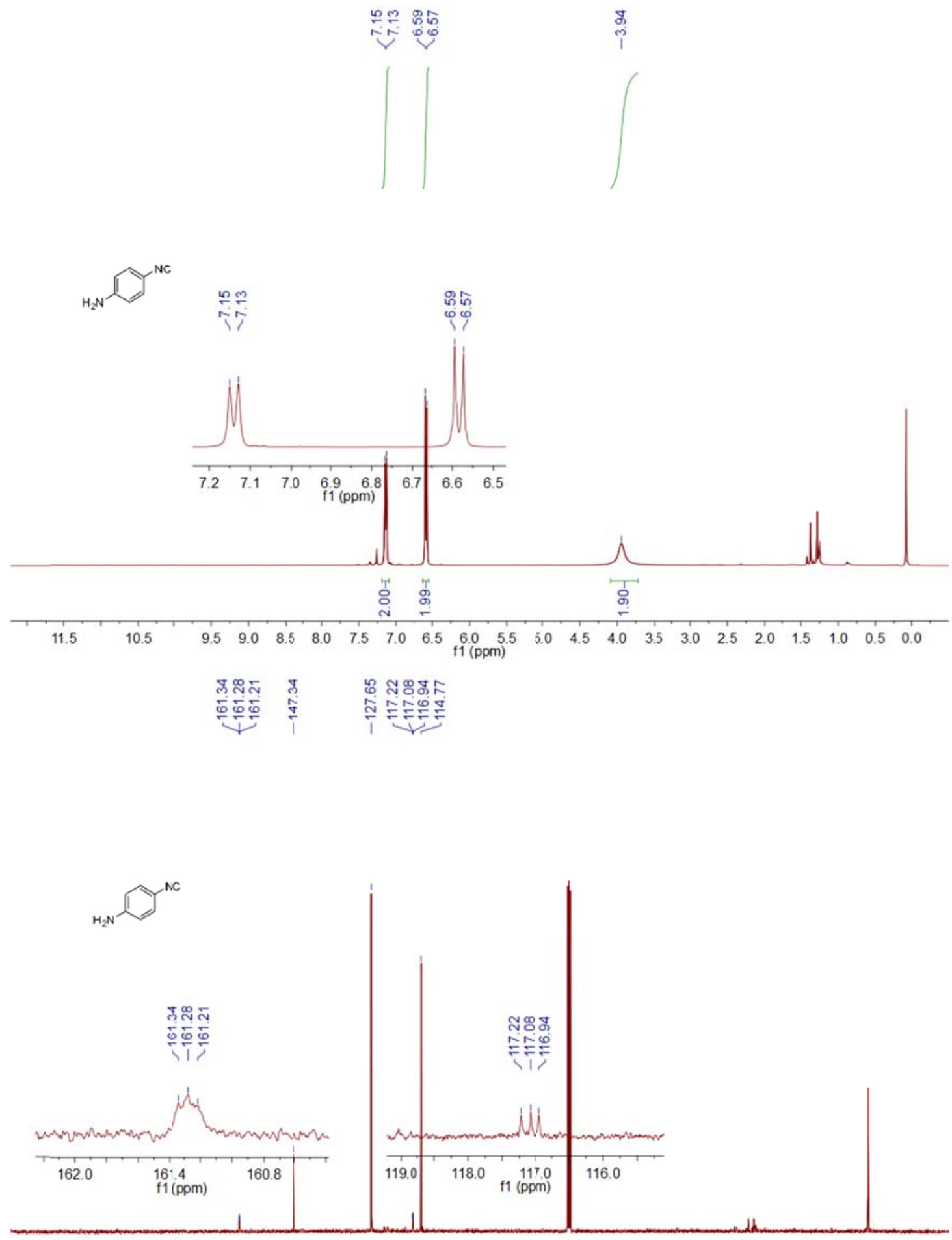

$\begin{array}{lllllllllllllllllllllll}210 & 200 & 190 & 180 & 170 & 160 & 150 & 140 & 130 & 120 & 110 & 100 & 90 & 80 & 70 & 60 & 50 & 40 & 30 & 20 & 10 & 0 & -10\end{array}$ 
1-isocyano-4-phenoxybenzene (3e. ${ }^{1} \mathrm{H}$ NMR $400 \mathrm{MHz}, \mathrm{CDCl}_{3} ;{ }^{13} \mathrm{C} \mathrm{NMR} 101 \mathrm{MHz}, \mathrm{CDCl}_{3}$ ).

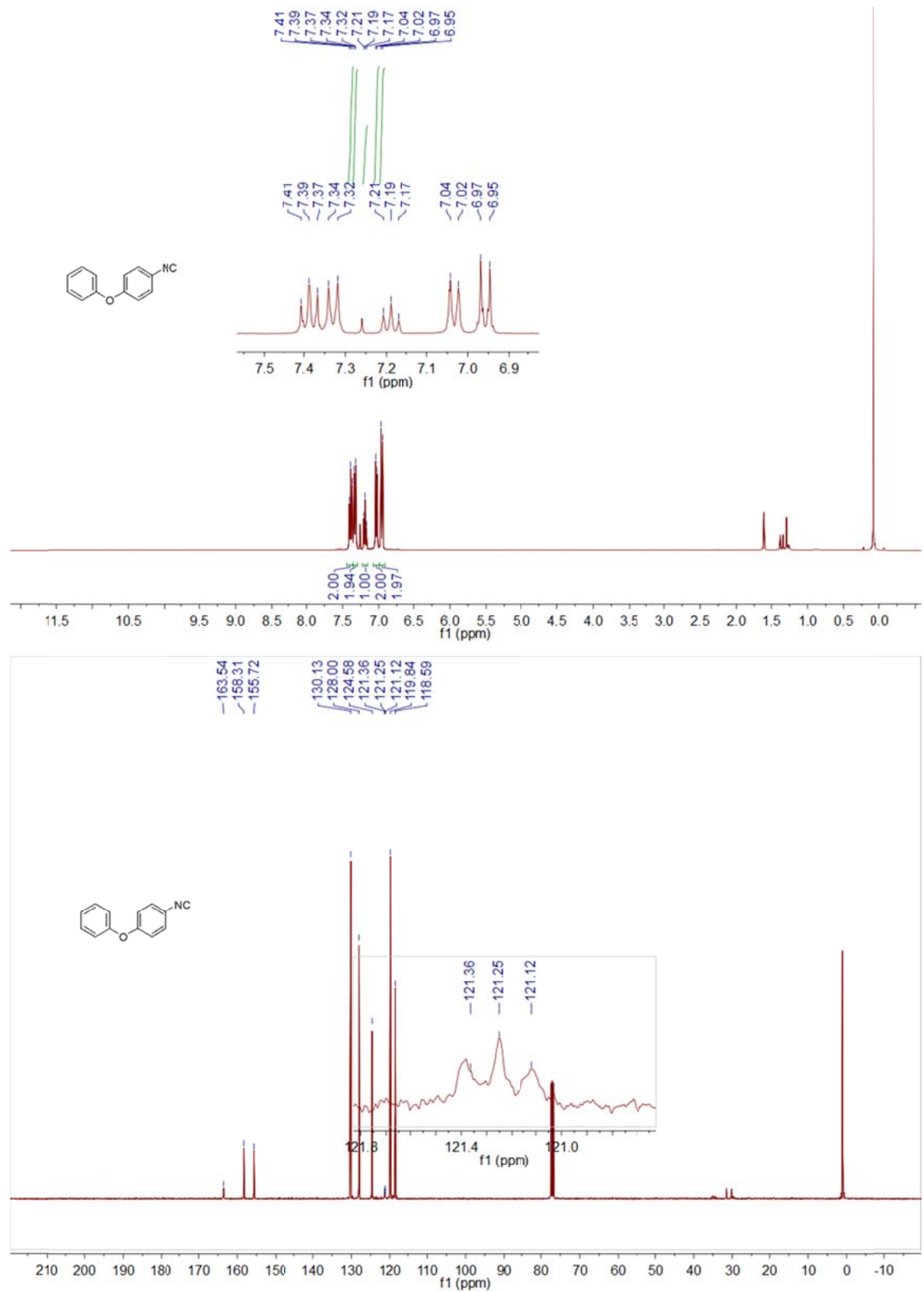


4,4'-oxybis(isocyanobenzene) (3f. ${ }^{1} \mathrm{H}$ NMR $400 \mathrm{MHz}, \mathrm{CDCl}_{3} ;{ }^{13} \mathrm{C} \mathrm{NMR} 101 \mathrm{MHz}, \mathrm{CDCl}_{3}$ ). 25L-5TX-76

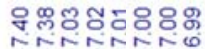
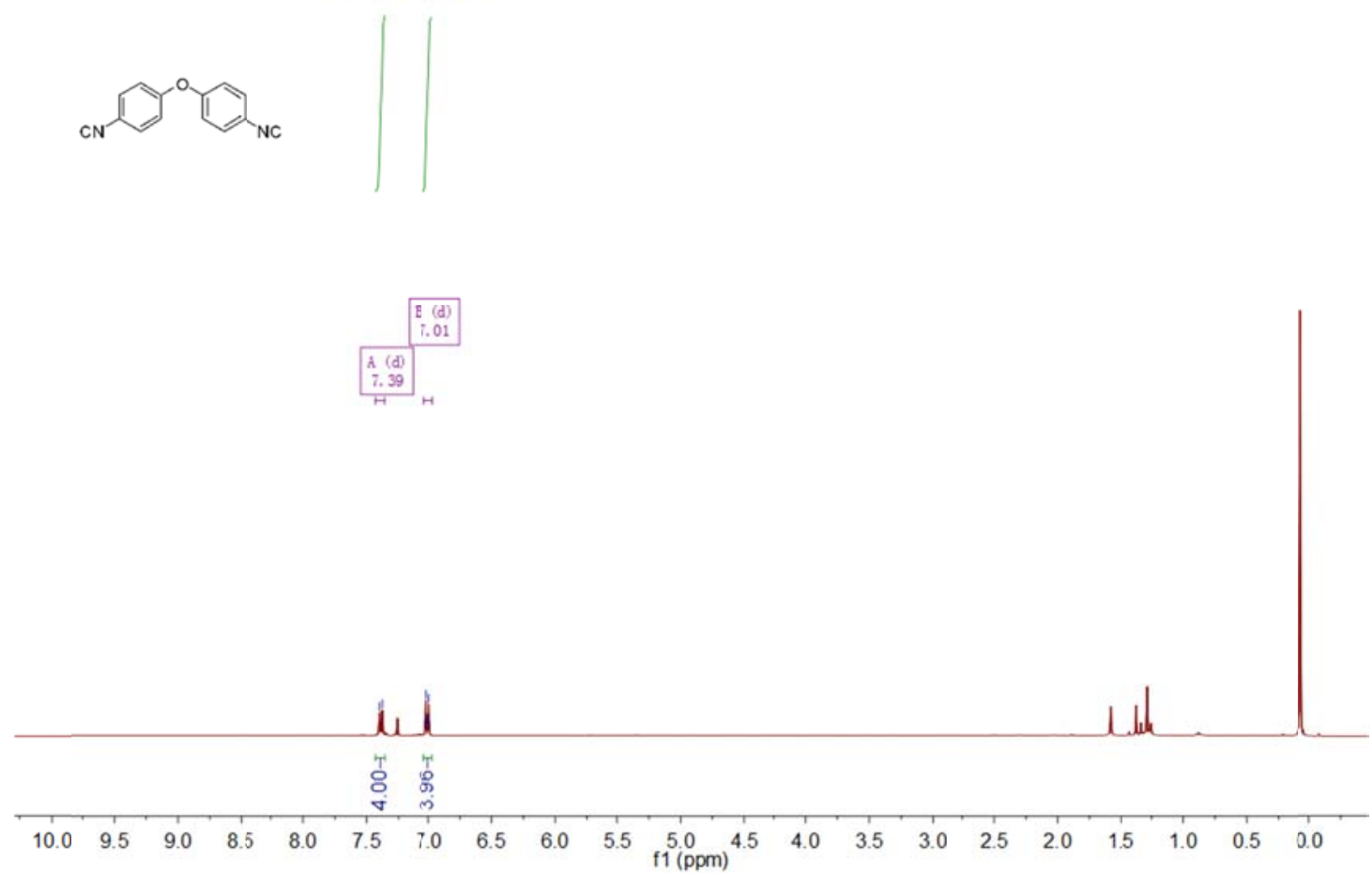

zSL-STX-76CD

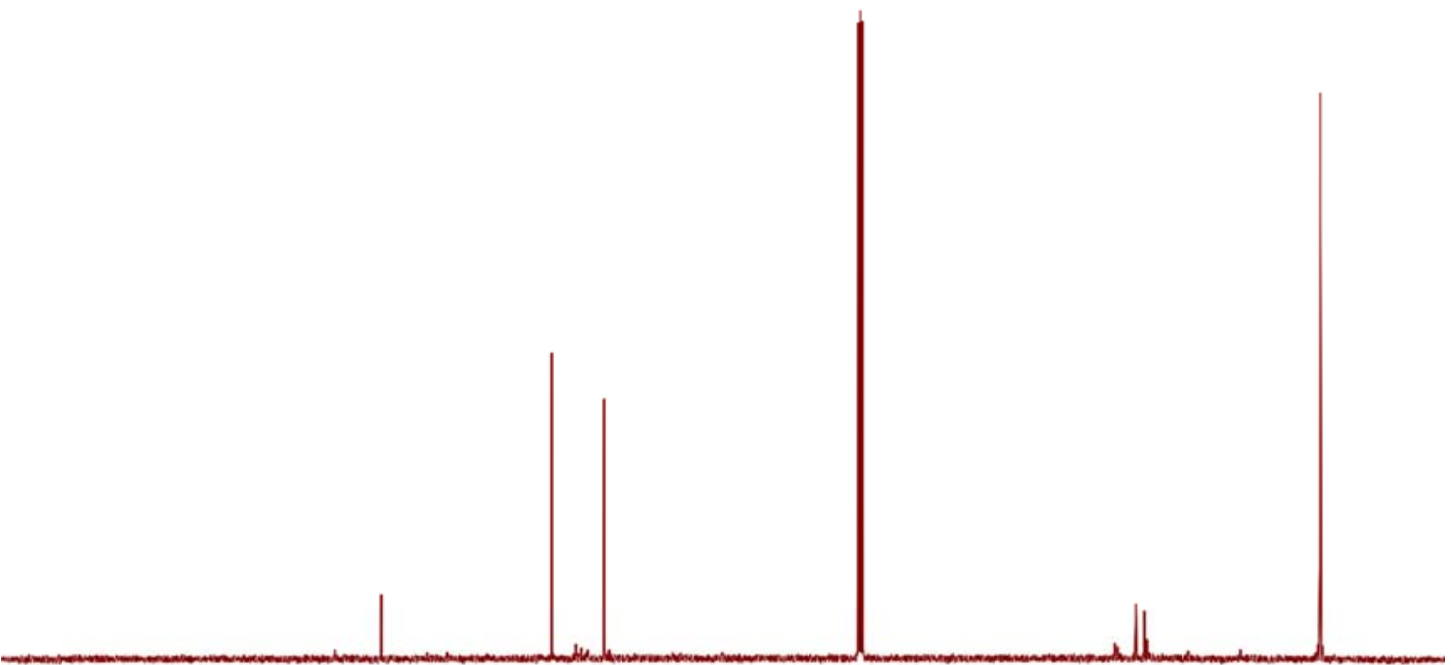

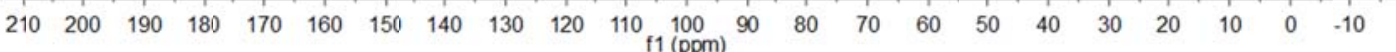


4-(4-isocyanophenoxy)aniline (3g. ${ }^{1} \mathrm{H}$ NMR $400 \mathrm{MHz}, \mathrm{CDCl}_{3} ;{ }^{13} \mathrm{C} \mathrm{NMR} 101 \mathrm{MHz}, \mathrm{CDCl}_{3}$ ).
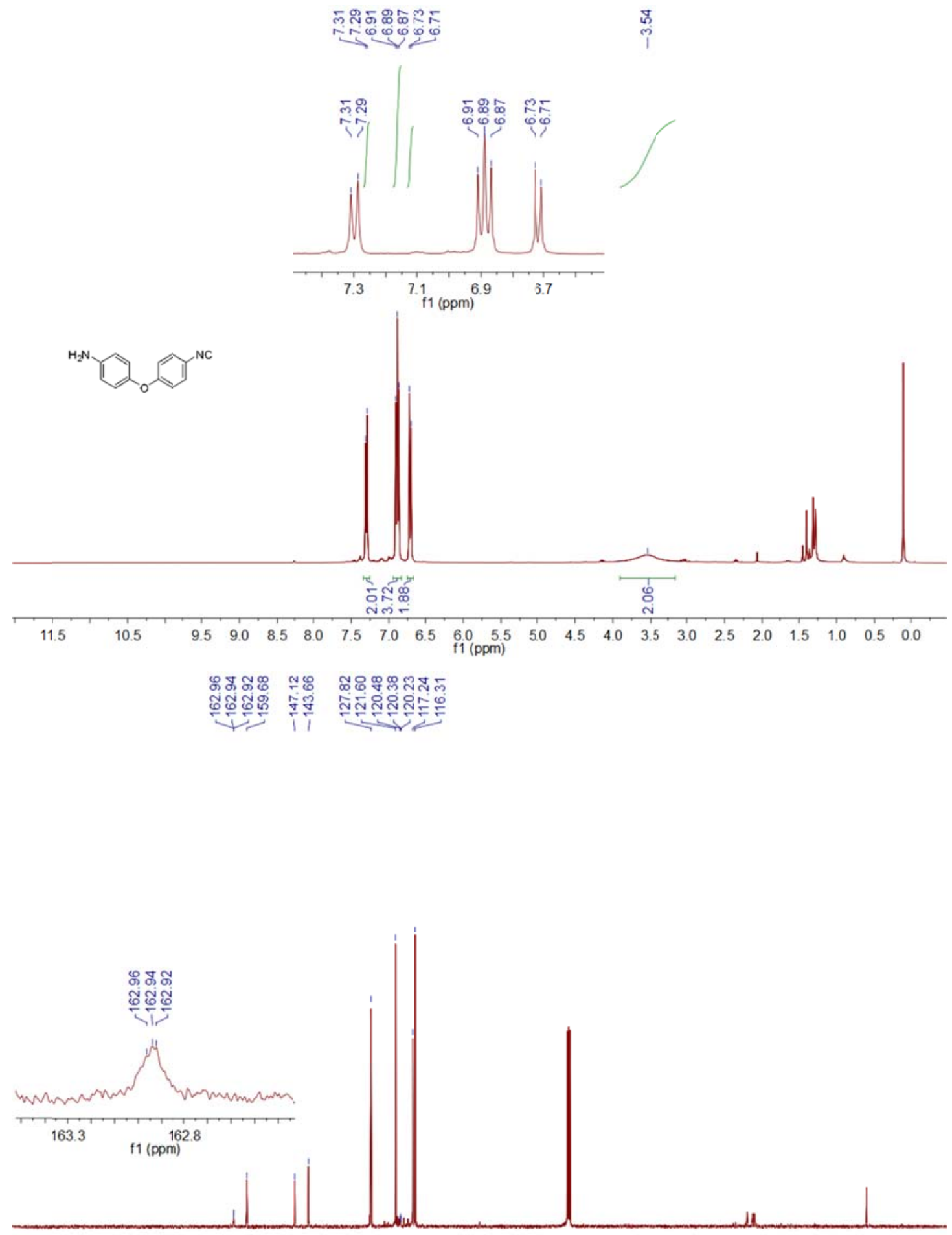

$\begin{array}{lllllllllllllllllllllll}210 & 200 & 190 & 180 & 170 & 160 & 150 & 140 & 130 & 120 & 110 & 100 & 90 & 80 & 70 & 60 & 50 & 40 & 30 & 20 & 10 & 0 & -10\end{array}$ 
4-isocyano-N-phenylaniline (3h. ${ }^{1} \mathrm{H}$ NMR $400 \mathrm{MHz}, \mathrm{CDCl}_{3} ;{ }^{13} \mathrm{C} \mathrm{NMR} 101 \mathrm{MHz}, \mathrm{CDCl}_{3}$ ).
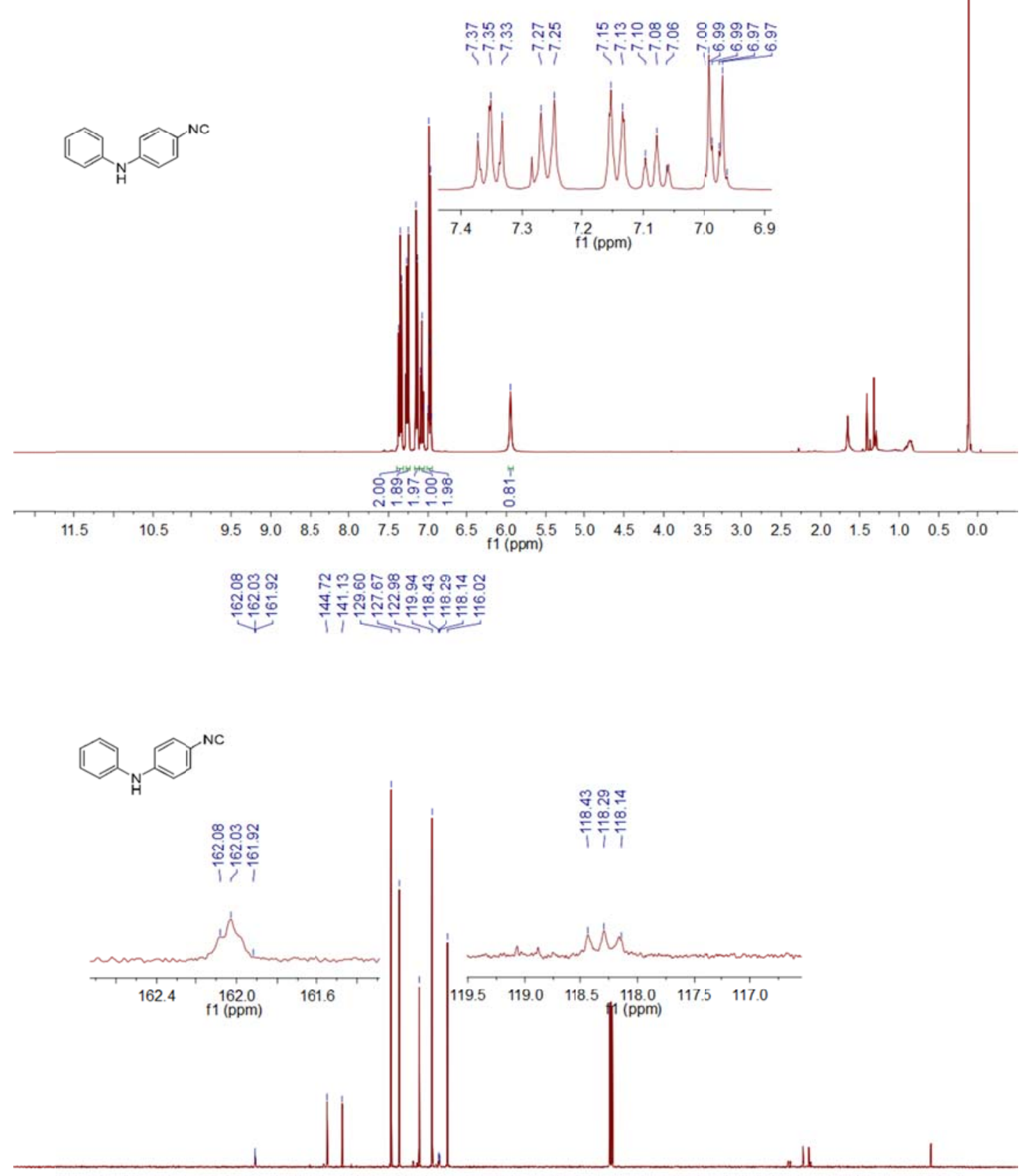

$\begin{array}{lllllllllllllllllllllll}210 & 200 & 190 & 180 & 170 & 160 & 150 & 140 & 130 & 120 & 110 & 100 & 90 & 80 & 70 & 60 & 50 & 40 & 30 & 20 & 10 & 0 & -10\end{array}$ 
N-(4-isocyanophenyl)acetamide (3i. ${ }^{1} \mathrm{H}$ NMR $400 \mathrm{MHz}$, DMSO-d $\mathrm{d}^{6} ;{ }^{13} \mathrm{C}$ NMR $101 \mathrm{MHz}$, DMSO-d $\left.{ }^{6}\right)$.
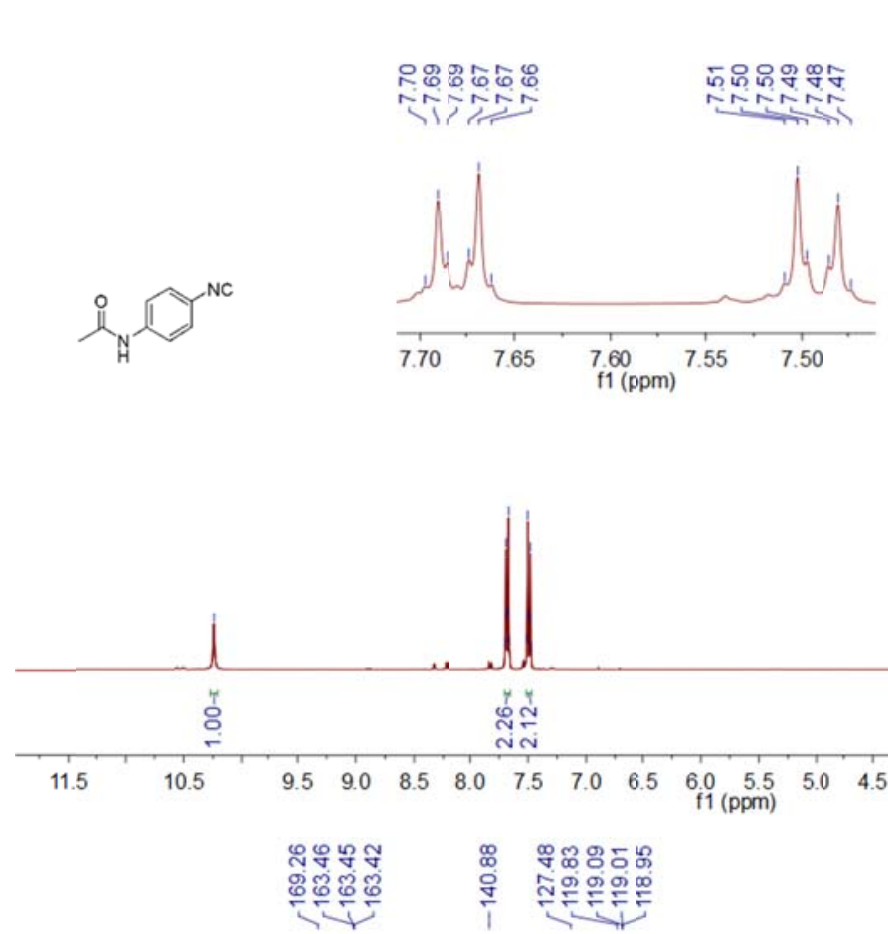

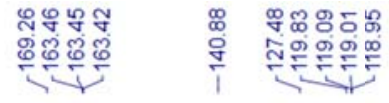

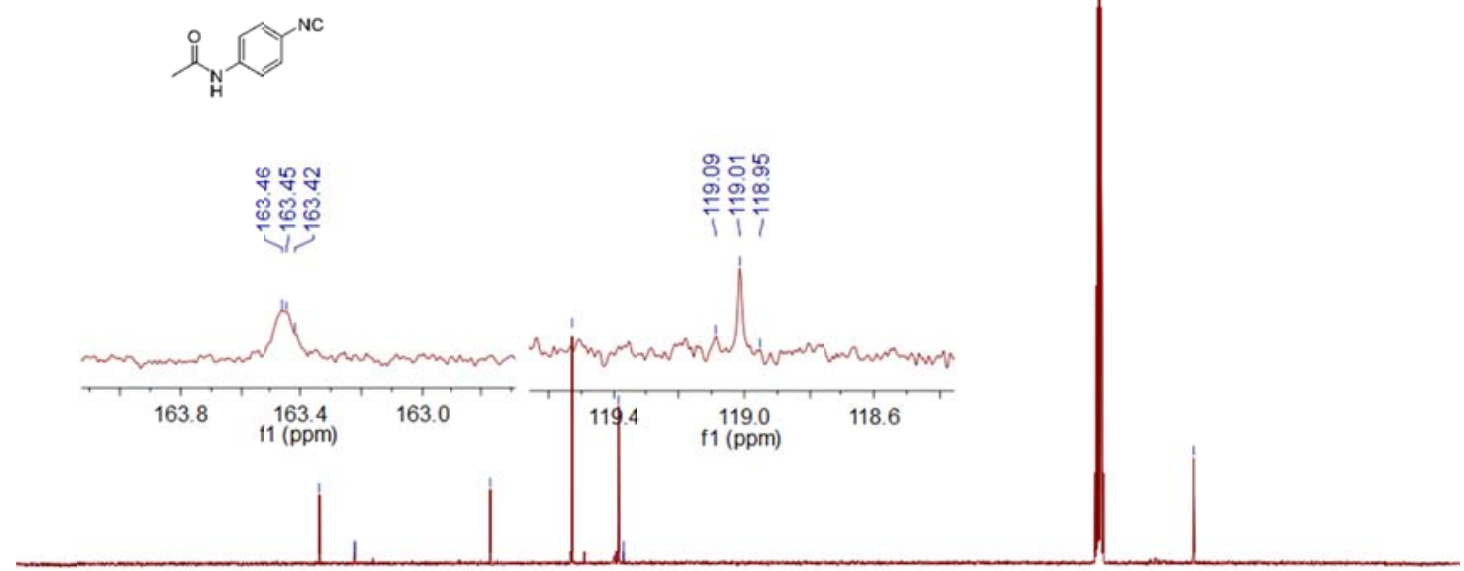

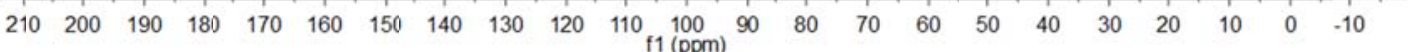


(4-isocyanophenyl)(methyl)sulfane (3j. ${ }^{1} \mathrm{H}$ NMR $400 \mathrm{MHz}, \mathrm{CDCl}_{3} ;{ }^{13} \mathrm{C}$ NMR $101 \mathrm{MHz}$, $\mathrm{CDCl}_{3}$ ).
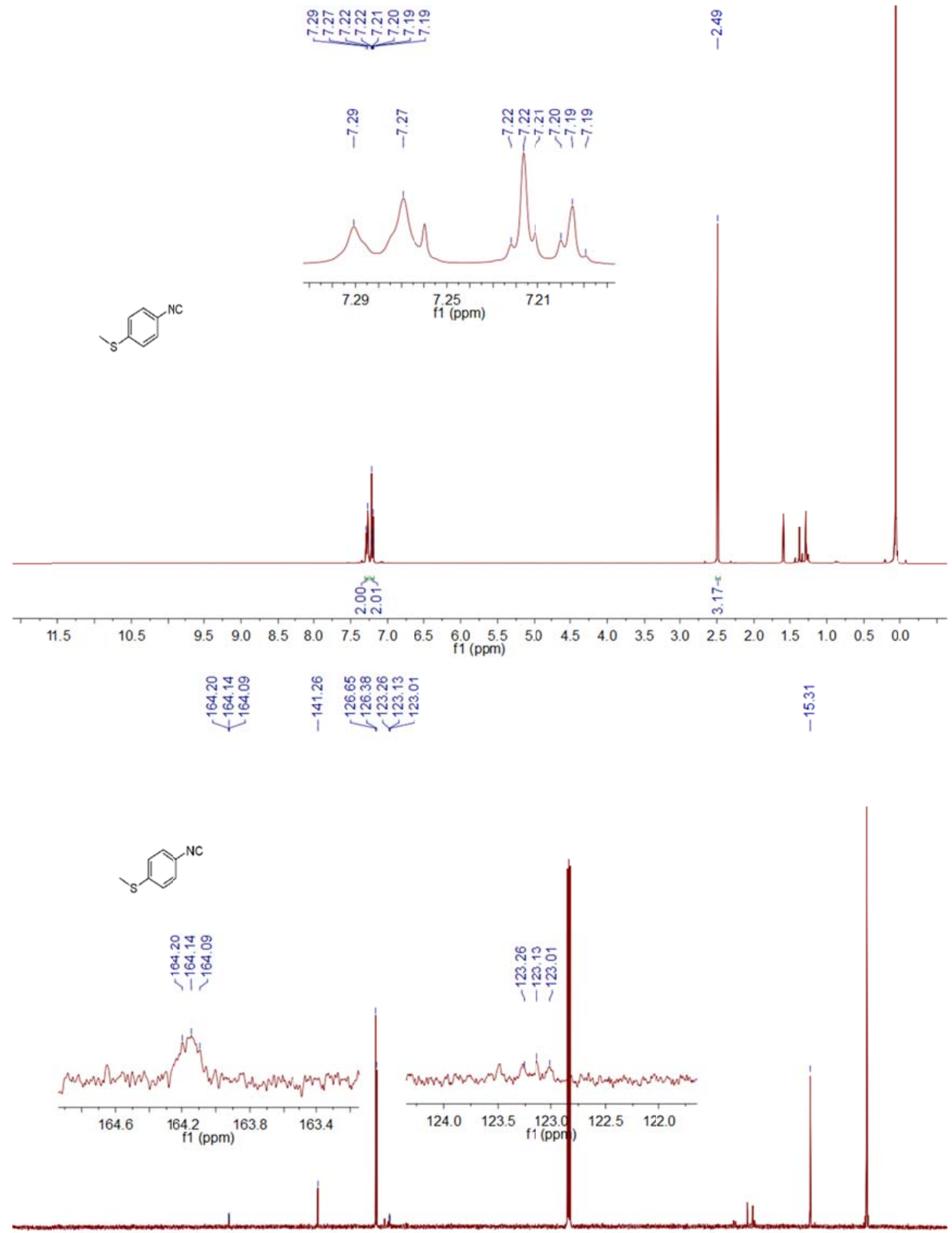

$\begin{array}{lllllllllllllllllllllll}210 & 200 & 190 & 180 & 170 & 160 & 150 & 140 & 130 & 120 & 110 & 100 & 90 & 80 & 70 & 60 & 50 & 40 & 30 & 20 & 10 & 0 & -10\end{array}$ 
1-ethoxy-4-isocyanobenzene (3k. ${ }^{1} \mathrm{H}$ NMR $400 \mathrm{MHz}, \mathrm{CDCl}_{3},{ }^{13} \mathrm{C}$ NMR $101 \mathrm{MHz}, \mathrm{CDCl}_{3}$ ).

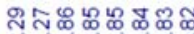

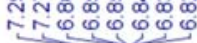

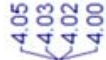

这方学
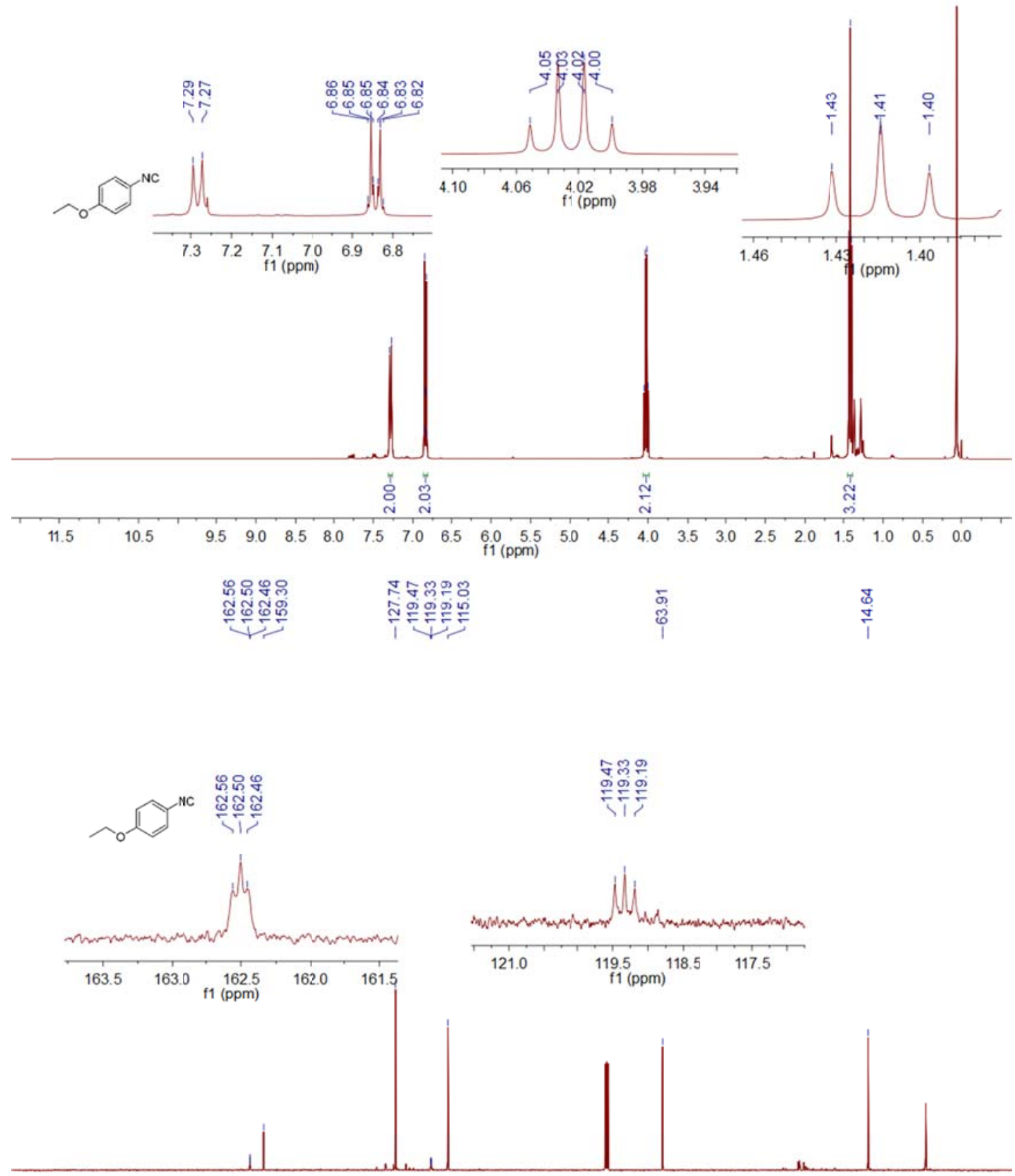

$\begin{array}{lllllllllllllllllllllll}210 & 200 & 190 & 180 & 170 & 160 & 150 & 140 & 130 & 120 & 110 & 100 & 90 & 80 & 70 & 60 & 50 & 40 & 30 & 20 & 10 & 0 & -10\end{array}$ 
(isocyanomethyl)benzene (3l. ${ }^{1} \mathrm{H}$ NMR $400 \mathrm{MHz}, \mathrm{CDCl}_{3} ;{ }^{13} \mathrm{C} \mathrm{NMR} 101 \mathrm{MHz}, \mathrm{CDCl}_{3}$ ).
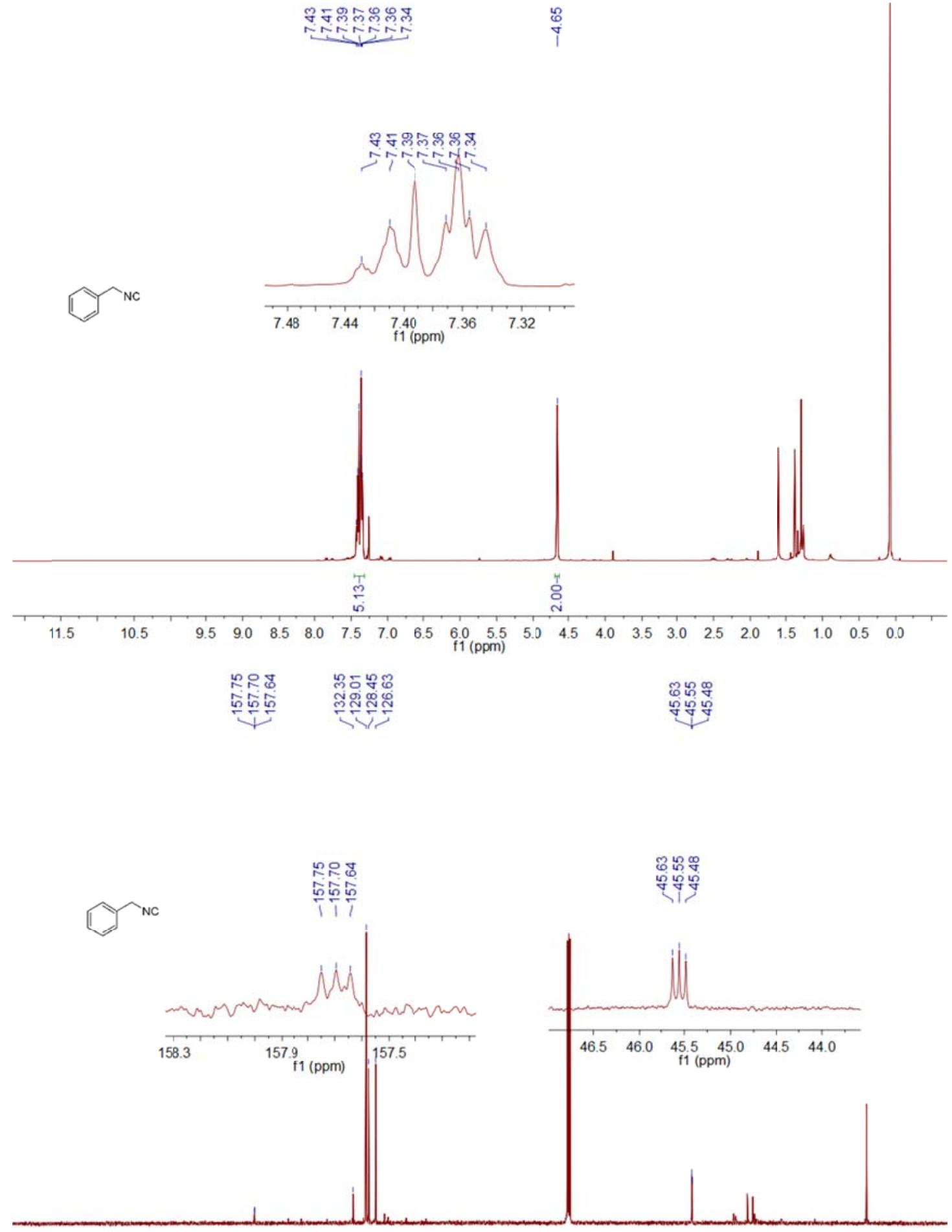

$\begin{array}{lllllllllllllllllllllll}210 & 200 & 190 & 180 & 170 & 160 & 150 & 140 & 130 & 120 & 110 & 100 & 90 & 80 & 70 & 60 & 50 & 40 & 30 & 20 & 10 & 0 & -10\end{array}$ 
1-(isocyanomethyl)-4-methoxybenzene (3m. ${ }^{1} \mathrm{H}$ NMR $400 \mathrm{MHz}, \mathrm{CDCl}_{3} ;{ }^{13} \mathrm{C} \mathrm{NMR} 101 \mathrm{MHz}$, $\left.\mathrm{CDCl}_{3}\right)$.

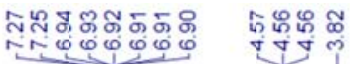
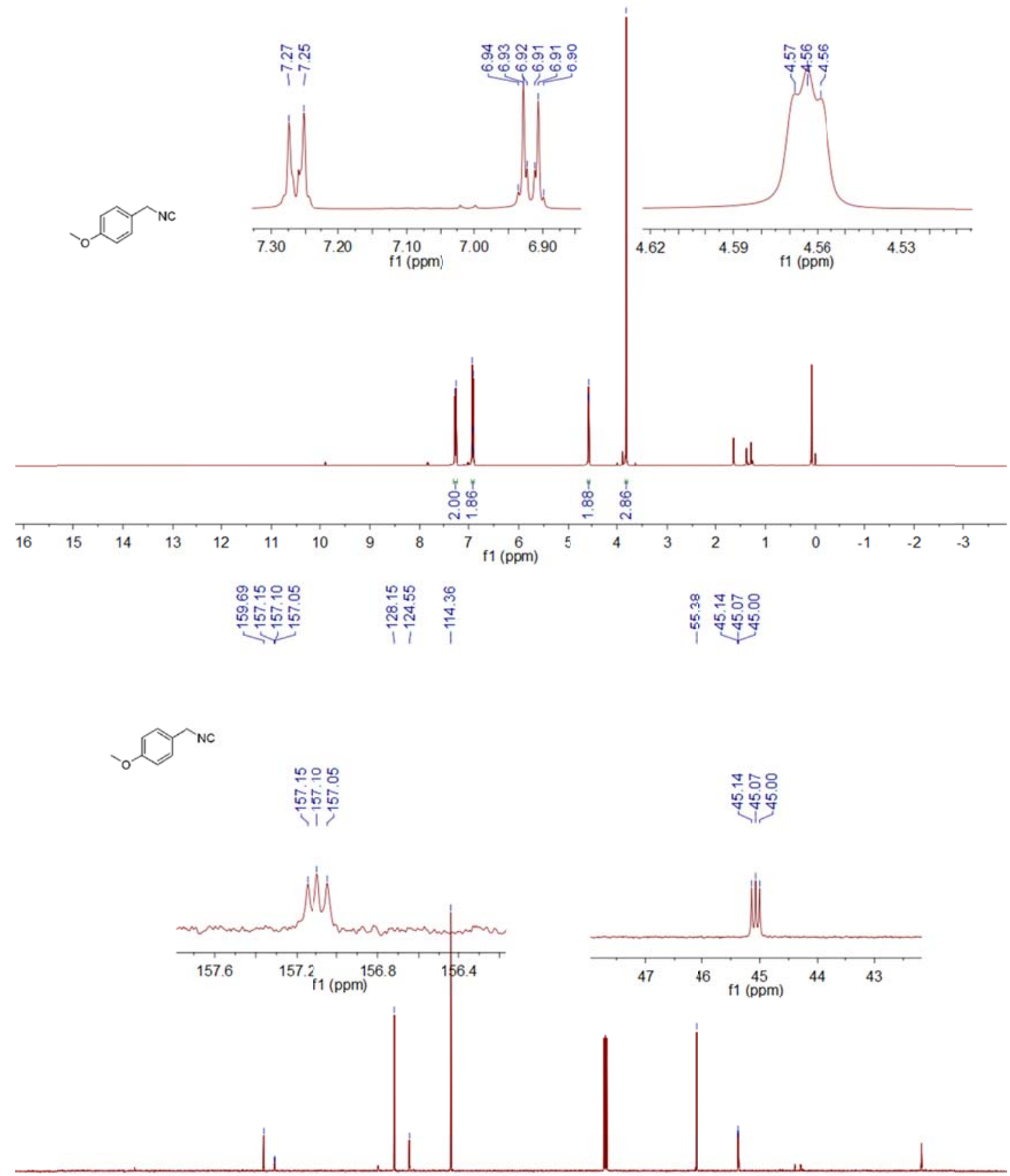

$\begin{array}{lllllllllllllllllllllll}210 & 200 & 190 & 180 & 170 & 160 & 150 & 140 & 130 & 120 & 110 \underset{\mathrm{f} 1(\mathrm{ppm})}{100} & 90 & 80 & 70 & 60 & 50 & 40 & 30 & 20 & 10 & 0 & -10\end{array}$ 
1-fluoro-4-(isocyanomethyl)benzene (3n. ${ }^{1} \mathrm{H}$ NMR $400 \mathrm{MHz}, \mathrm{CDCl}_{3} ;{ }^{13} \mathrm{C}$ NMR $101 \mathrm{MHz}$, $\left.\mathrm{CDCl}_{3}\right)$.

2SL-SYX-86
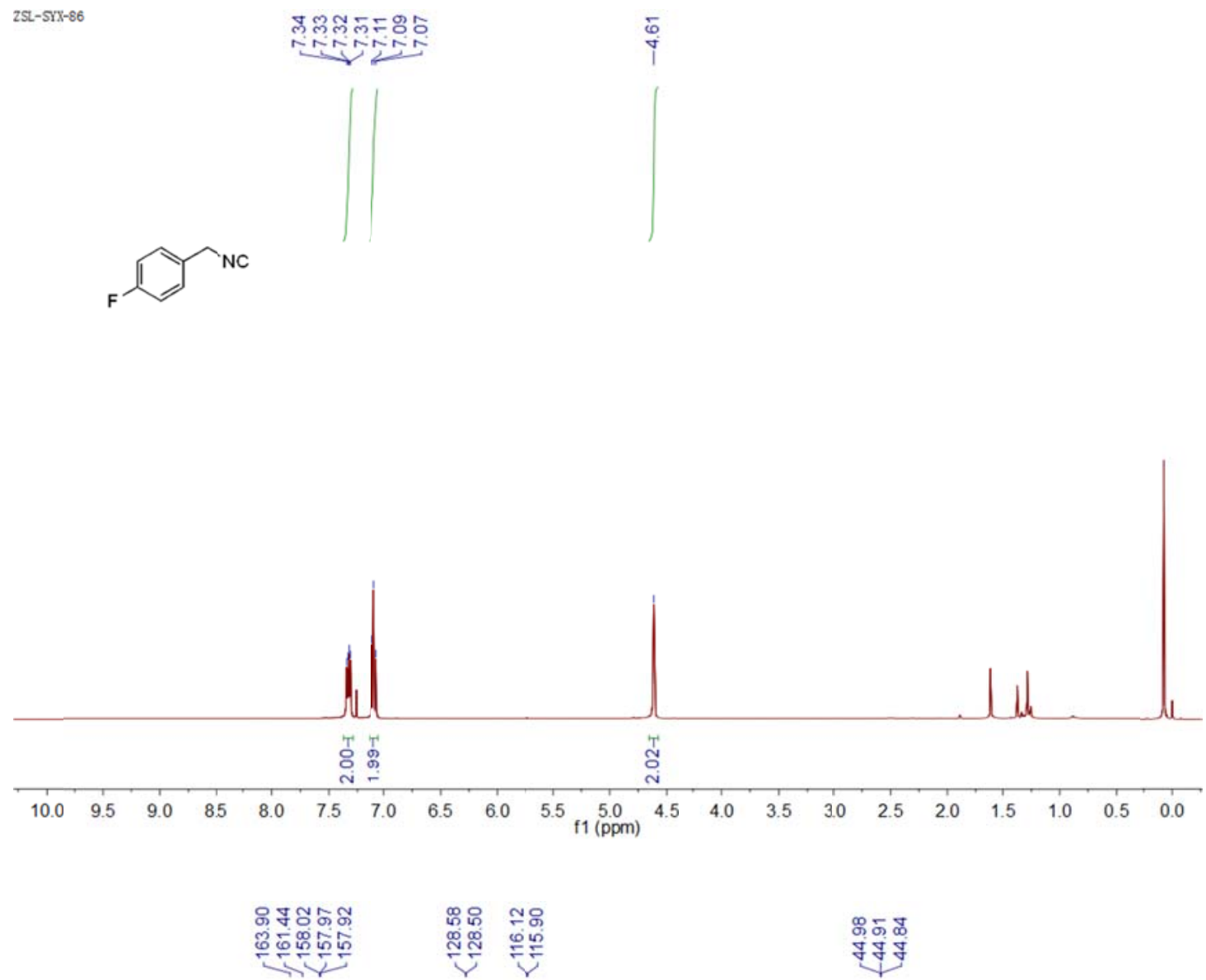

\section{ஜூர்}

过昌

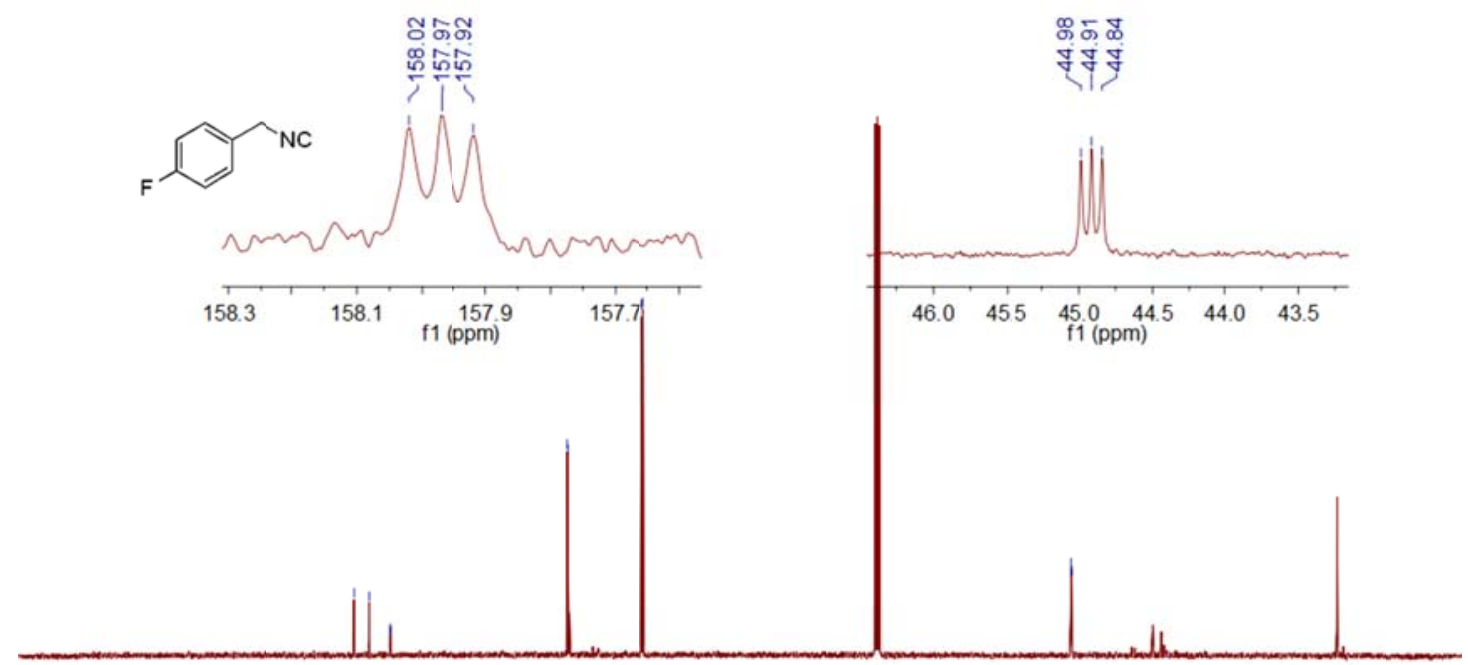

$\begin{array}{lllllllllllllllllllllll}210 & 200 & 190 & 180 & 170 & 160 & 150 & 140 & 130 & 120 & 110 & \begin{array}{l}100 \\ \mathrm{f} 1(\mathrm{ppm})\end{array} & 90 & 80 & 70 & 60 & 50 & 40 & 30 & 20 & 10 & 0 & -10\end{array}$ 
1-(isocyanomethyl)-3-methylbenzene (3o. ${ }^{1} \mathrm{H}$ NMR $400 \mathrm{MHz}, \mathrm{CDCl}_{3} ;{ }^{13} \mathrm{C}$ NMR $101 \mathrm{MHz}$, $\left.\mathrm{CDCl}_{3}\right)$.

\begin{tabular}{|c|}
\hline 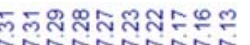 \\
\hline
\end{tabular}
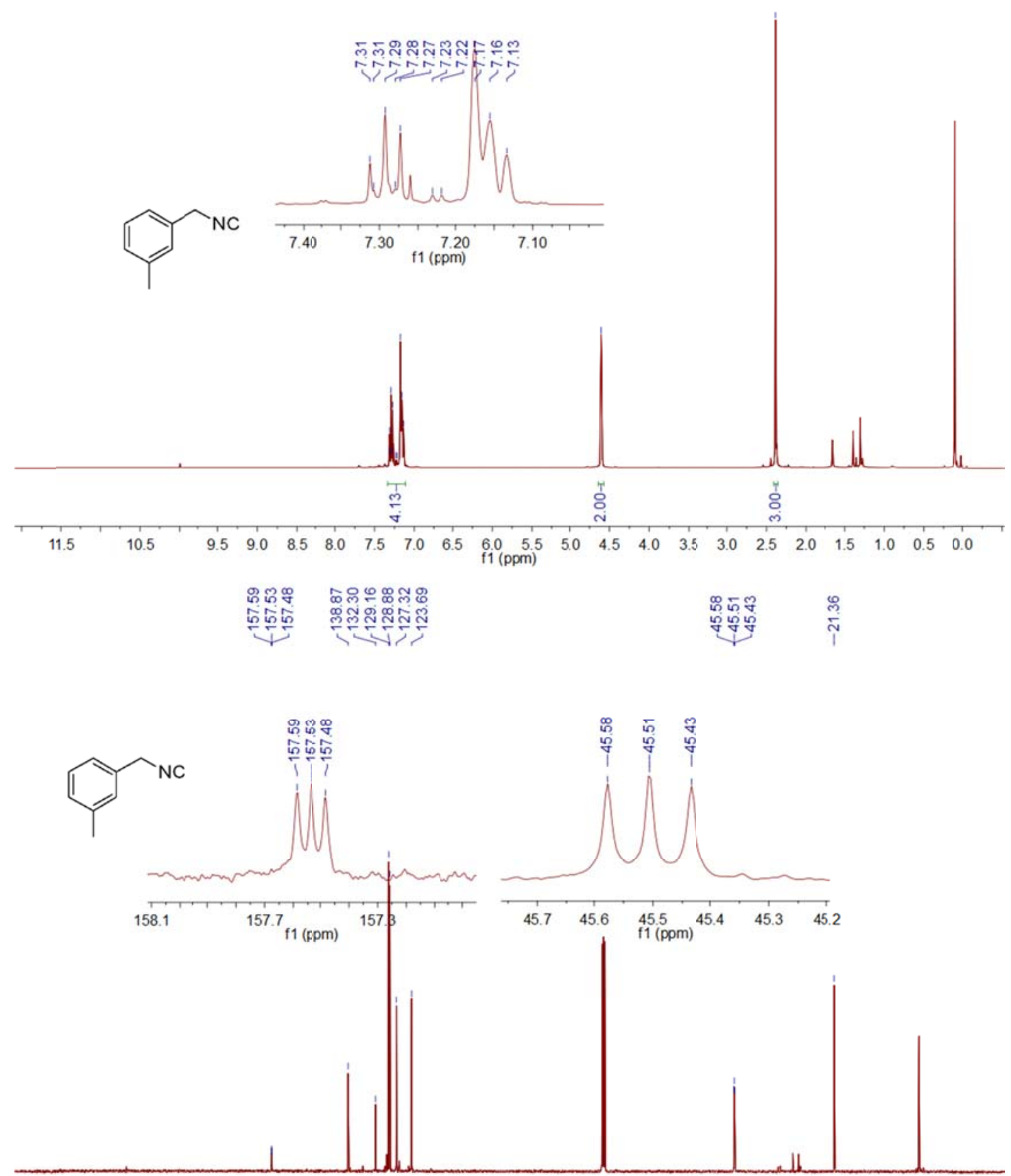

$\begin{array}{lllllllllllllllllllllll}210 & 200 & 190 & 180 & 170 & 160 & 150 & 140 & 130 & 120 & 110 & 100 & 90 & 80 & 70 & 60 & 50 & 40 & 30 & 20 & 10 & 0 & -10\end{array}$ 
2-(isocyanomethyl)furan (3p. ${ }^{1} \mathrm{H} \mathrm{NMR} 400 \mathrm{MHz}, \mathrm{CDCl}_{3} ;{ }^{13} \mathrm{C} \mathrm{NMR} 101 \mathrm{MHz}, \mathrm{CDCl}_{3}$ ).
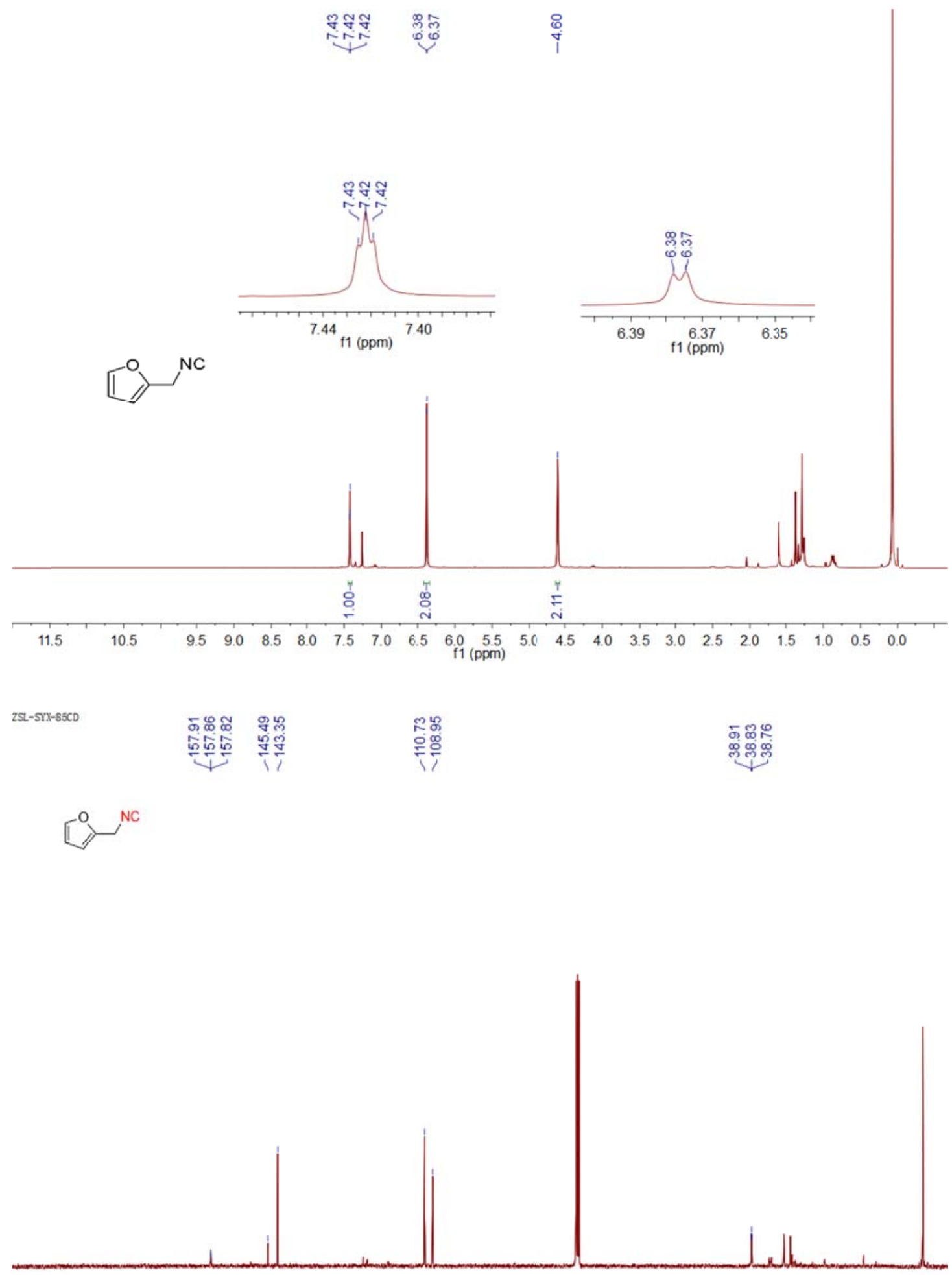

$\begin{array}{lllllllllllllllllllll}200 & 190 & 180 & 170 & 160 & 150 & 140 & 130 & 120 & 110 & 100 & 90 & 80 & 70 & 60 & 50 & 40 & 30 & 20 & 10 & 0\end{array}$ 
(S)-(1-isocyanoethyl)benzene (3q. ${ }^{1} \mathrm{H}$ NMR $400 \mathrm{MHz}, \mathrm{CDCl}_{3} ;{ }^{13} \mathrm{C} \mathrm{NMR} 101 \mathrm{MHz}, \mathrm{CDCl}_{3}$ ).

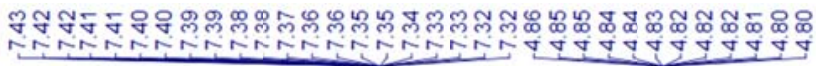

우우영용요
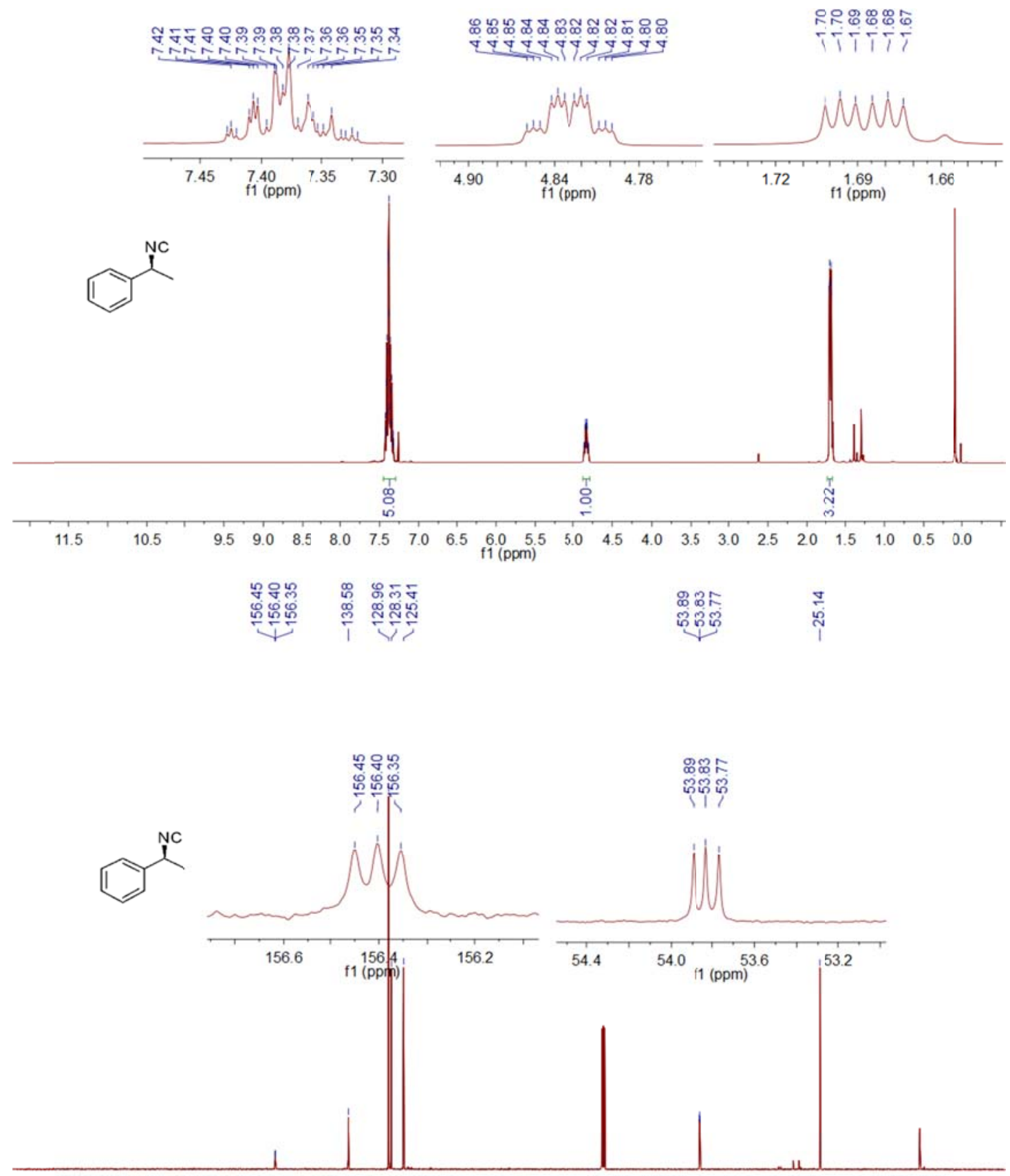

$\begin{array}{lllllllllllllllllllllll}210 & 200 & 190 & 180 & 170 & 160 & 150 & 140 & 130 & 120 & 110 & \begin{array}{l}100 \\ \mathrm{f} 1(\mathrm{ppm})\end{array} & 90 & 80 & 70 & 60 & 50 & 40 & 30 & 20 & 10 & 0 & -10\end{array}$ 
(2-isocyanoethyl)benzene (3r. ${ }^{1} \mathrm{H}$ NMR $400 \mathrm{MHz}, \mathrm{CDCl}_{3} ;{ }^{13} \mathrm{C} \mathrm{NMR} 101 \mathrm{MHz}, \mathrm{CDCl}_{3}$ ). 2SL-SYX-78-1

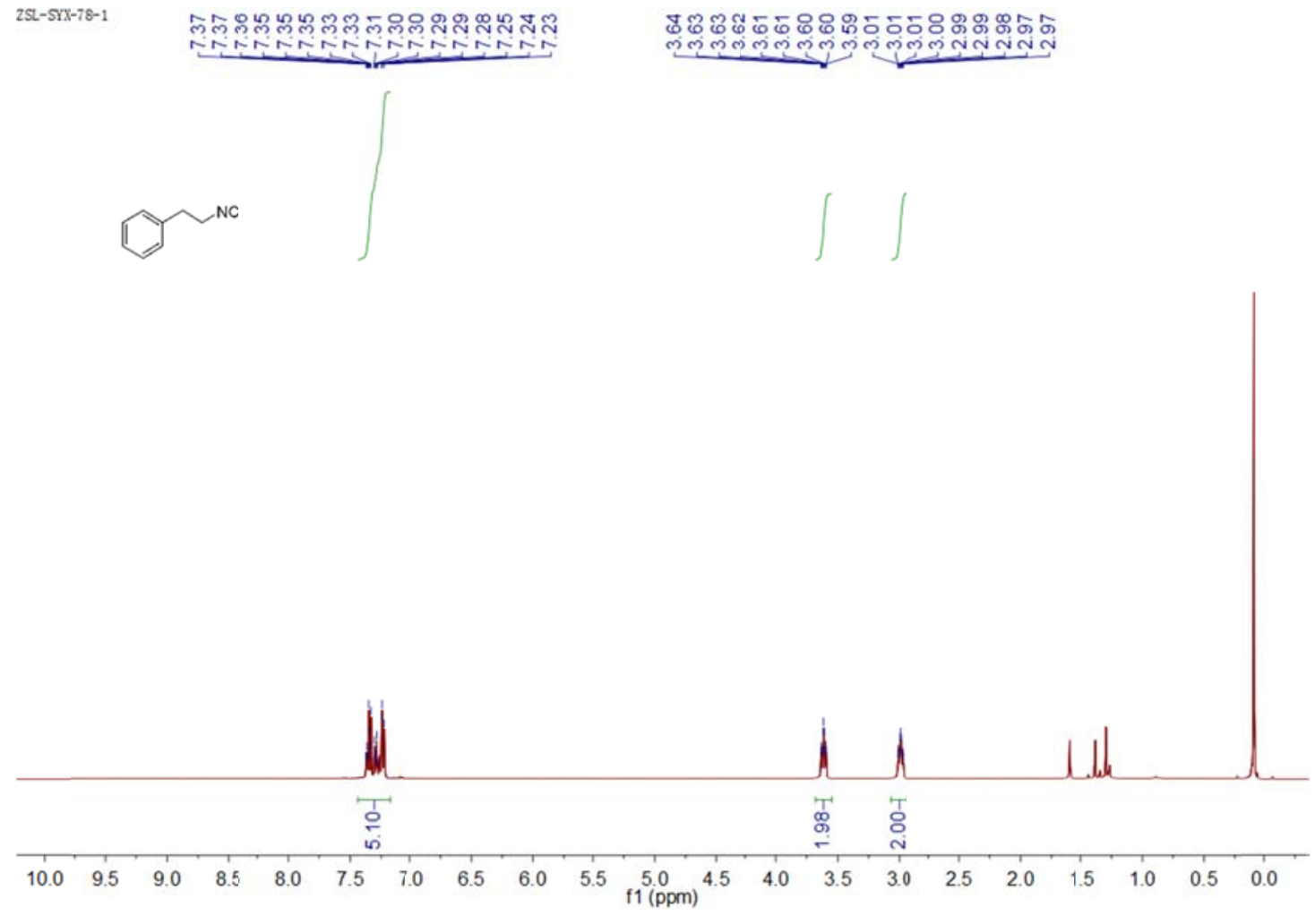

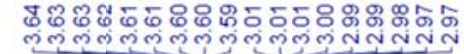

$2 S L-S Y X-7 @ D$

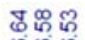

때요요

는

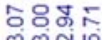

ขูงู่ติ
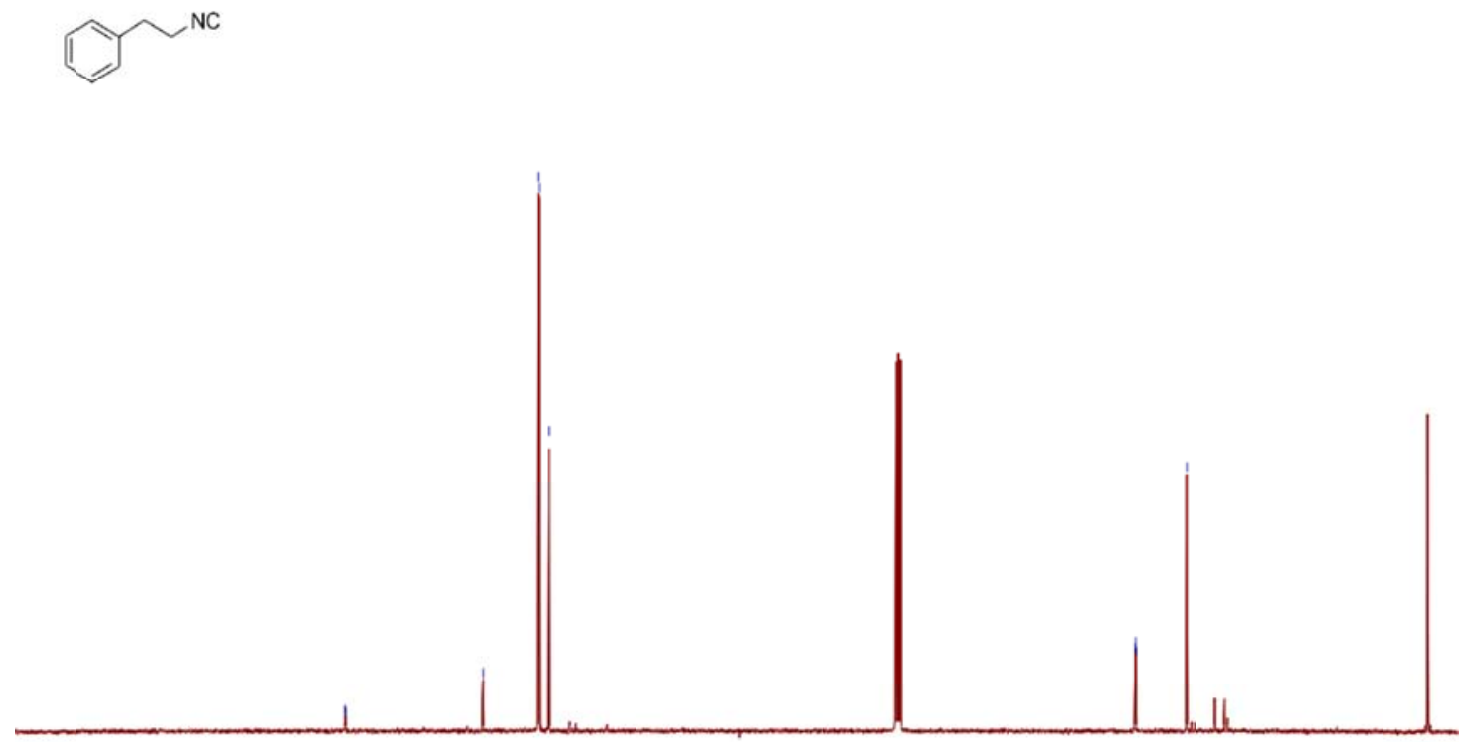

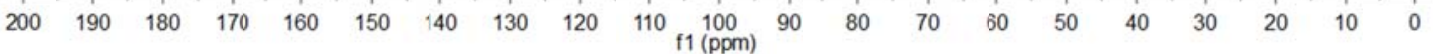


1-(2-isocyanoethyl)-4-methoxybenzene (3s. ${ }^{1} \mathrm{H}$ NMR $400 \mathrm{MHz}, \mathrm{CDCl}_{3} ;{ }^{13} \mathrm{C}$ NMR $101 \mathrm{MHz}$, $\left.\mathrm{CDCl}_{3}\right)$.

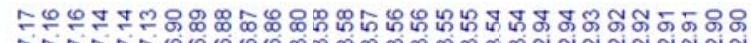

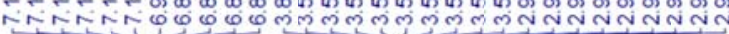
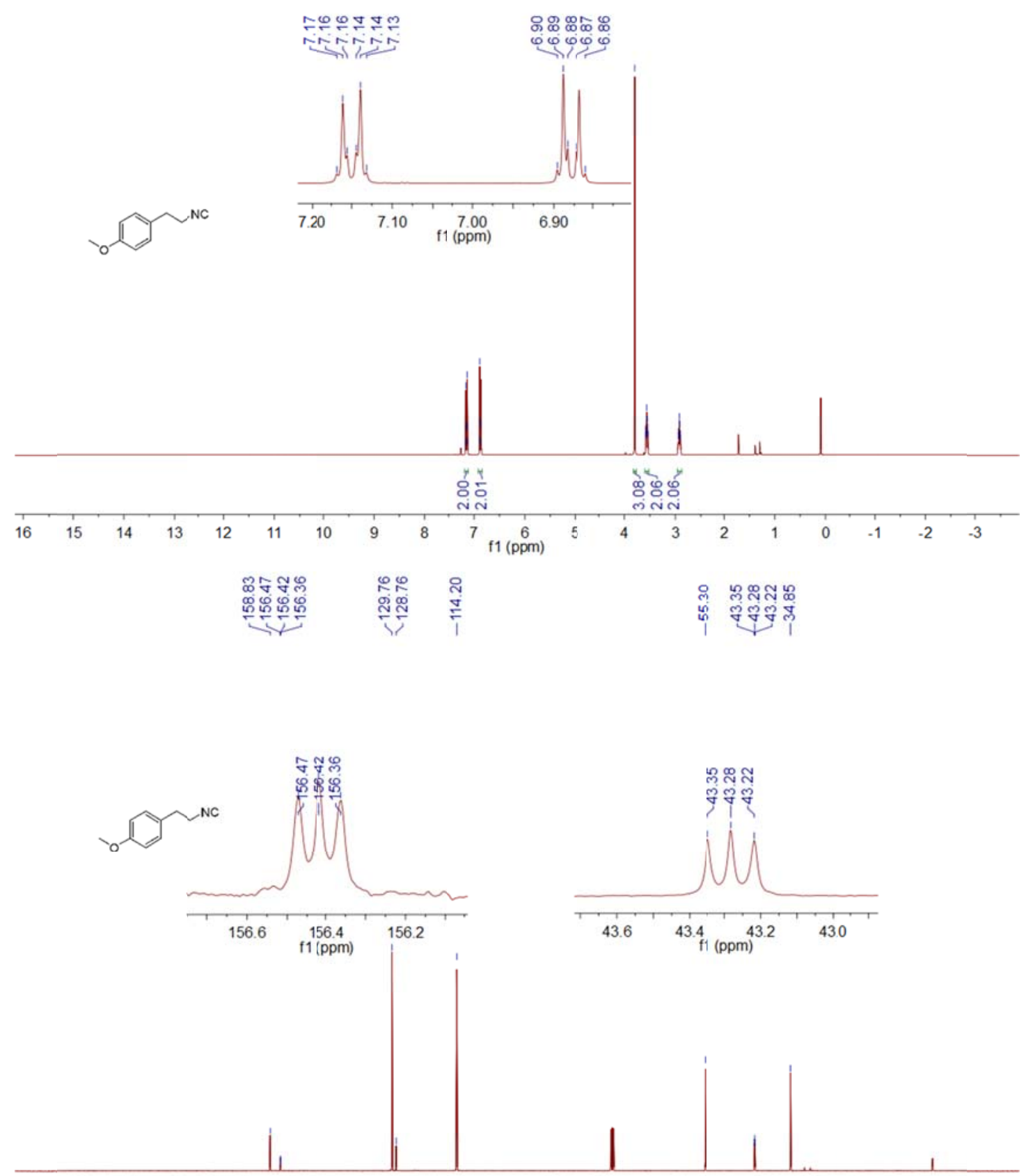

$\begin{array}{lllllllllllllllllllllll}210 & 200 & 190 & 180 & 170 & 160 & 150 & 140 & 130 & 120 & 110 & 100 & 90 & 80 & 70 & 60 & 50 & 40 & 30 & 20 & 10 & 0 & -10\end{array}$ 
(3-isocyanobutyl)benzene (3t. ${ }^{1} \mathrm{H}$ NMR $400 \mathrm{MHz}, \mathrm{CDCl}_{3} ;{ }^{13} \mathrm{C} \mathrm{NMR} 101 \mathrm{MHz}, \mathrm{CDCl}_{3}$ ).

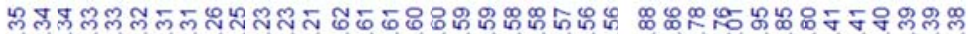

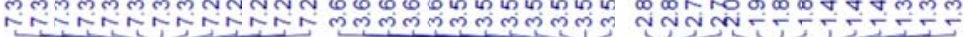
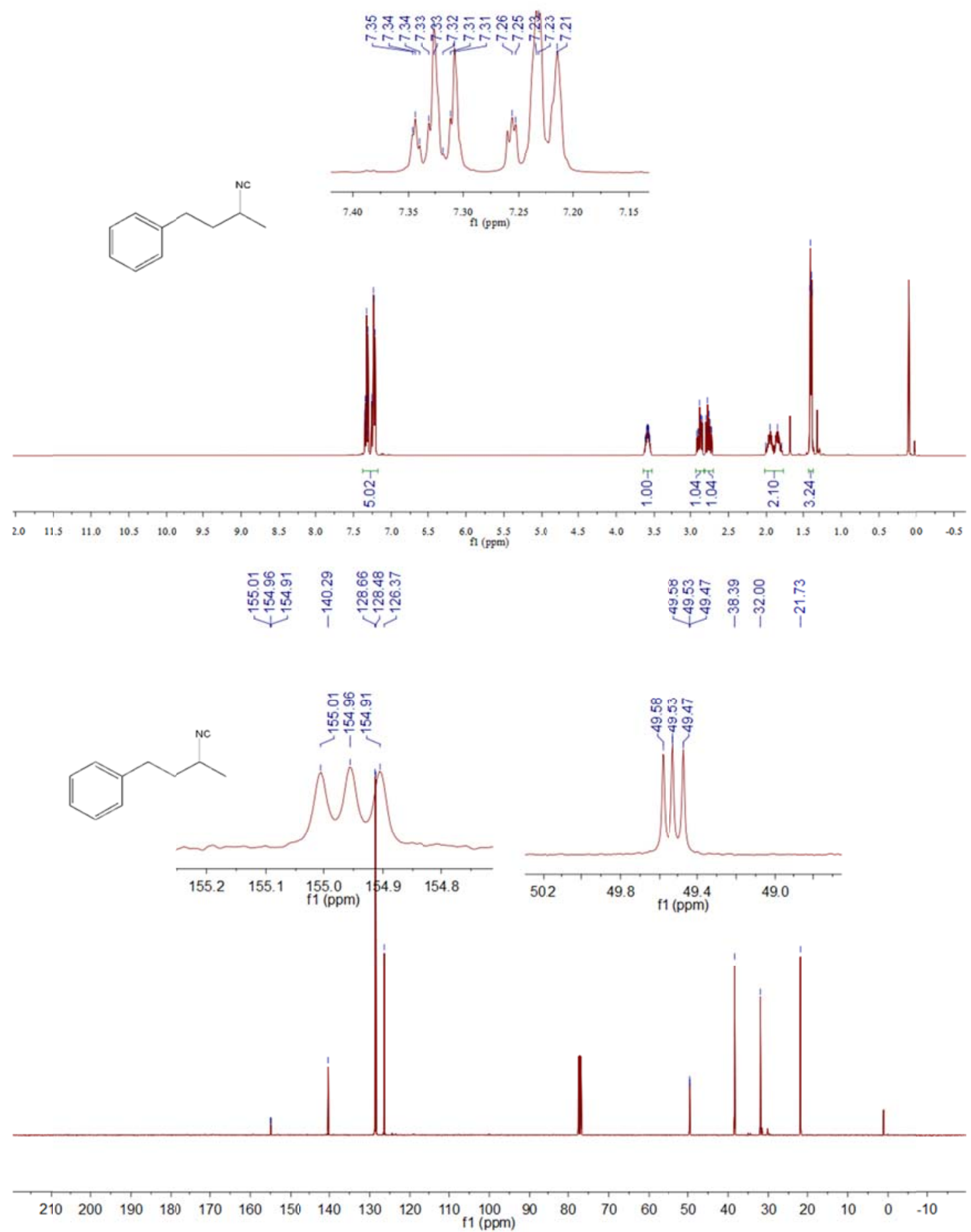
(2-isocyanopropan-2-yl)benzene (3u. ${ }^{1} \mathrm{H}$ NMR $400 \mathrm{MHz}, \mathrm{CDCl}_{3} ;{ }^{13} \mathrm{C} \mathrm{NMR} 101 \mathrm{MHz}, \mathrm{CDCl}_{3}$ ). 2SL-STX-92

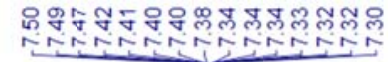<smiles>CC(C)(C)c1ccccc1</smiles>

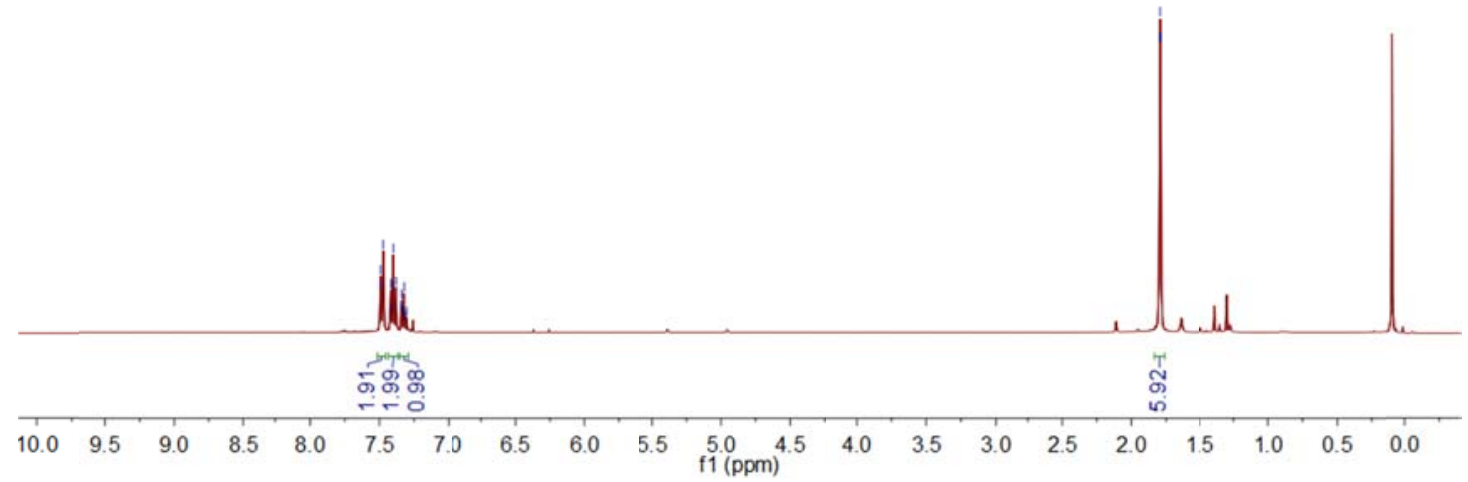

$2 S L-S T X-92 C D$

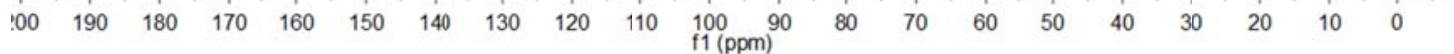


4-(2-isocyanoethyl)aniline (3v. ${ }^{1} \mathrm{H} \mathrm{NMR} 400 \mathrm{MHz}, \mathrm{CDCl}_{3} ;{ }^{13} \mathrm{C} \mathrm{NMR} 101 \mathrm{MHz}, \mathrm{CDCl}_{3}$ ).

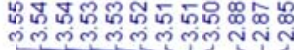

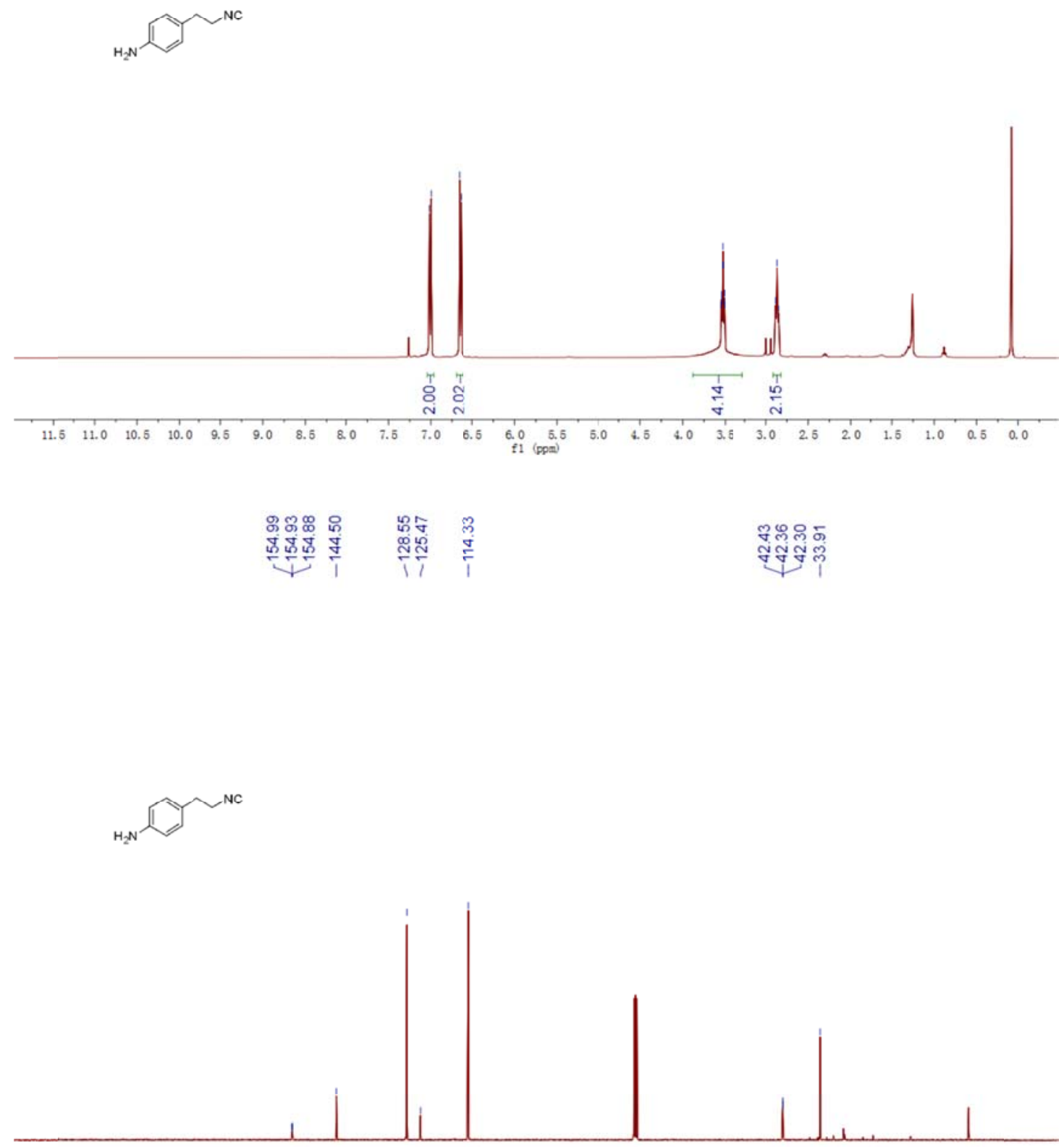

$210 \quad 200 \quad 190 \quad 180 \quad 170 \quad 160 \quad 150 \quad 140 \quad 130 \quad 120 \quad 110 \quad 100 \quad 90 \quad 80 \quad 70 \quad 60 \quad 50 \quad 40 \quad 30 \quad 20 \quad 10 \quad 0 \quad-10 \quad-2$ 
4-(2-chlorophenyl)-2-((2-isocyanoethoxy)methyl)-6-methyl-1,4-dihydropyridine-3,5-dicarbox ylate (3x. HRMS (ESI); ${ }^{1} \mathrm{H}$ NMR $400 \mathrm{MHz}, \mathrm{CDCl}_{3} ;{ }^{13} \mathrm{C} \mathrm{NMR} 101 \mathrm{MHz}, \mathrm{CDCl}_{3}$ ).

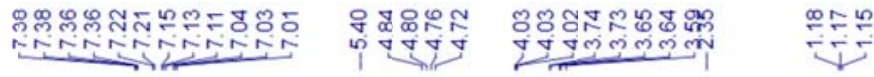
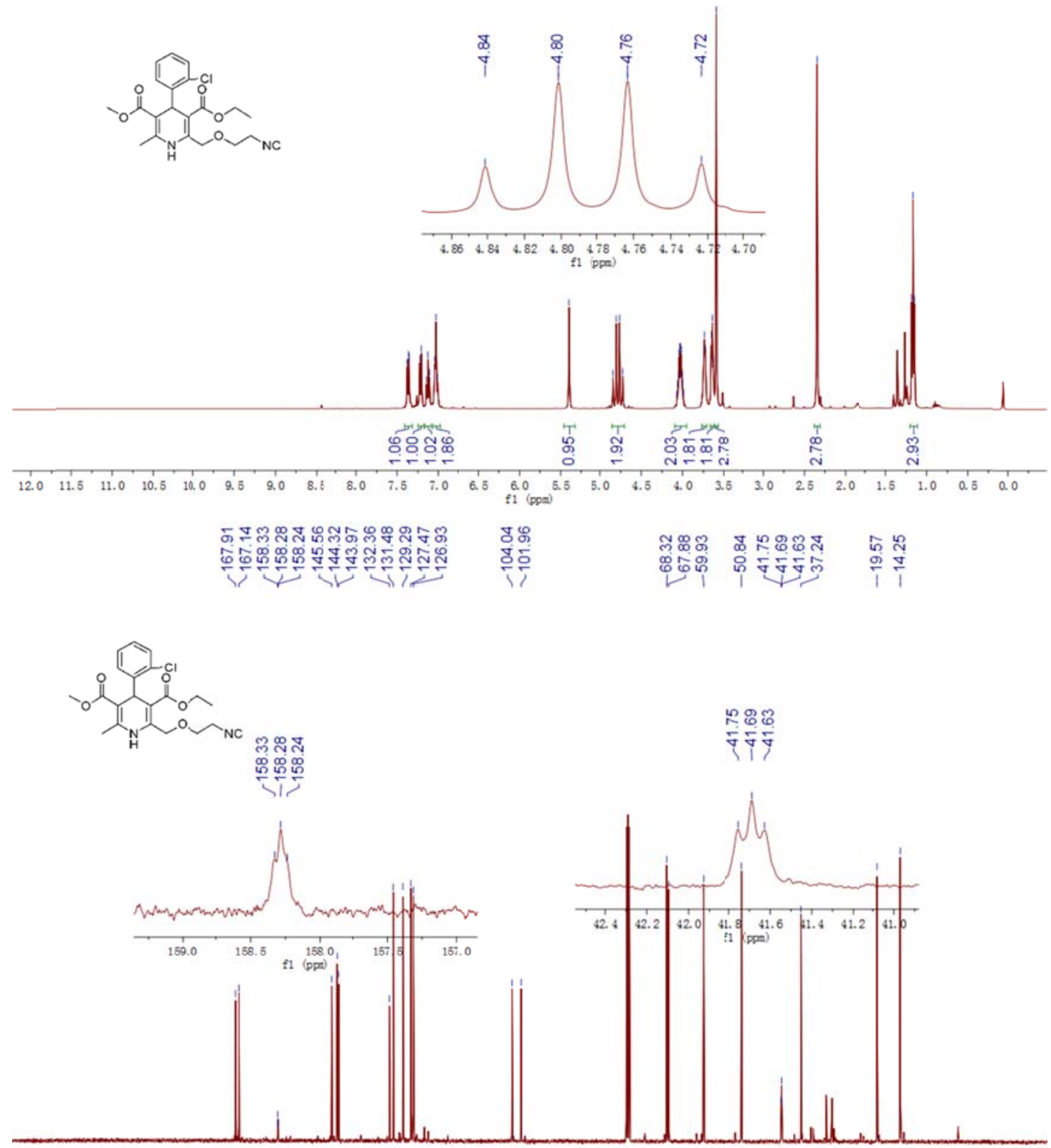

$\begin{array}{llllllllllll}210 & 200 & 190 & 180 & 170 & 160 & 150 & 140 & 130 & 120 & 110 & 100\end{array}$ 
1-25 \#16 RT: $0.10 \quad$ AV: $1 \quad$ NL: 1.55E9

T: FTMS + p ESI Full lock ms [80.0000-1200.0000]

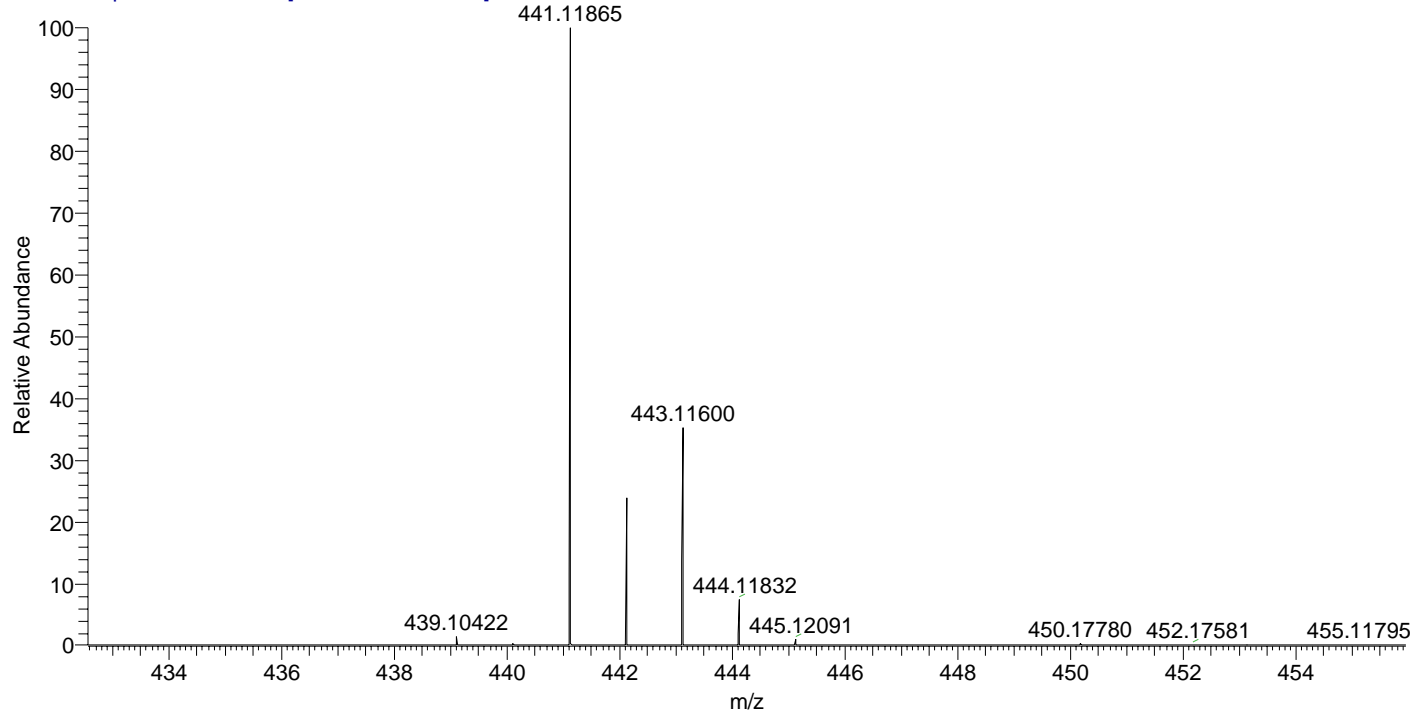


4-(4-(isocyanomethyl)phenyl)morpholine (3y. ${ }^{1} \mathrm{H}$ NMR $400 \mathrm{MHz}, \mathrm{CDCl}_{3} ;{ }^{13} \mathrm{C}$ NMR $101 \mathrm{MHz}$, $\left.\mathrm{CDCl}_{3}\right)$.

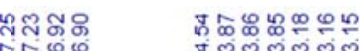

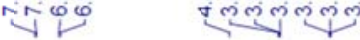
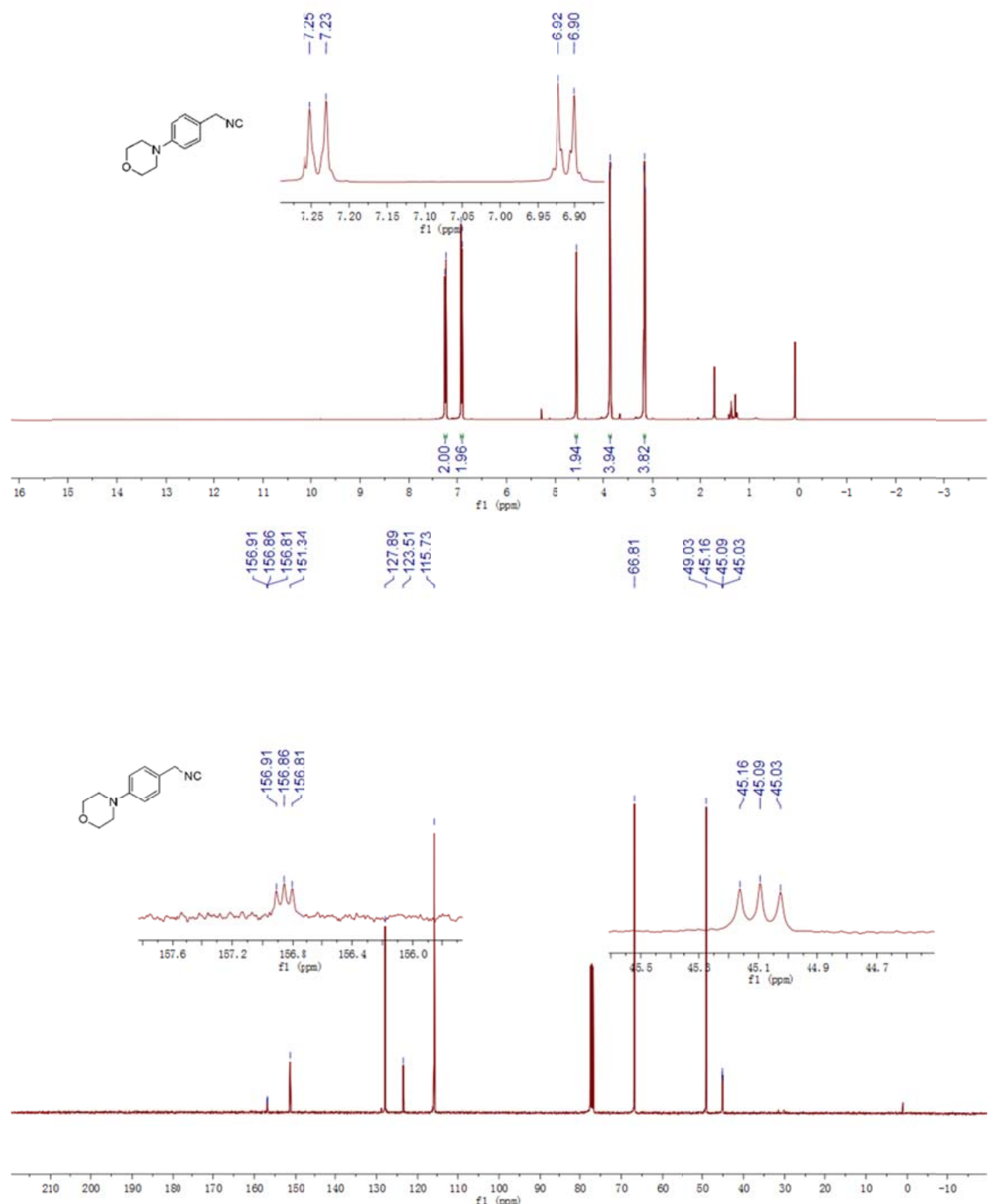
(1R,4aS,10aR)-1-(isocyanomethyl)-7-isopropyl-1,4a-dimethyl-1,2,3,4,4a,9,10,10a-octahydrop henanthrene (3z. HRMS (ESI); ${ }^{1} \mathrm{H}$ NMR $400 \mathrm{MHz}, \mathrm{CDCl}_{3} ;{ }^{13} \mathrm{C} \mathrm{NMR} 101 \mathrm{MHz}, \mathrm{CDCl}_{3}$ ).

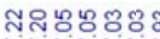

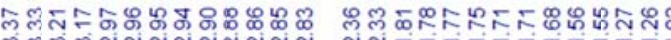

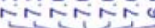
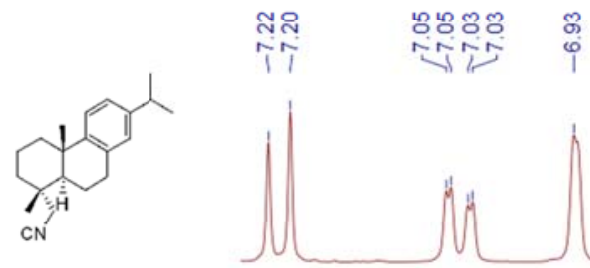

$\begin{array}{lllllllll}7.20 & 7.15 & 7.10 & 7.06 & 7.00 & 6.96 & 6.90 & 6.85 & 6.80\end{array}$
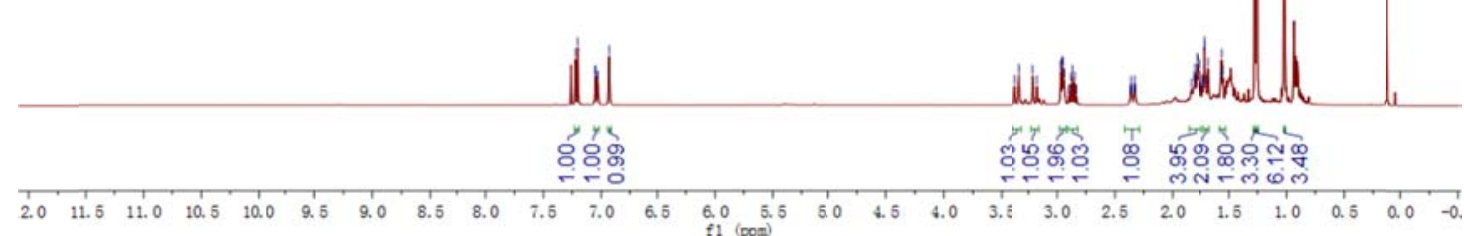

$\begin{array}{llllllllllllllllllllllllll}2.0 & 11.5 & 11.0 & 10.5 & 10.0 & 9.5 & 9.0 & 8.5 & 8.0 & 7.5 & 7.0 & 6.5 & 6.0 & 5.5 & 5.0 & 4.5 & 4.0 & 3.6 & 3.0 & 2.5 & 2.0 & 1.5 & 1.0 & 0.5 & 0.0 & -0.0\end{array}$

ل

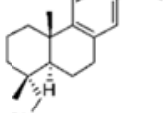

cí

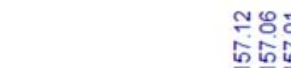

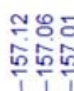

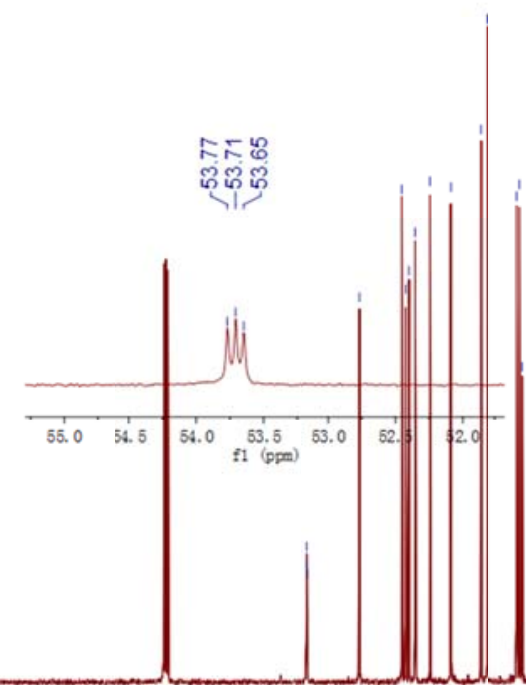

$210 \quad 200 \quad 190 \quad 190$

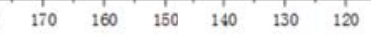

$110 \underset{f 1}{100}(\mathrm{ppm})$ 
1-26 \#19 RT: $0.11 \quad$ AV: 1 NL: 4.96E8

T: FTMS + p ESI Full lock ms [80.0000-1200.0000]

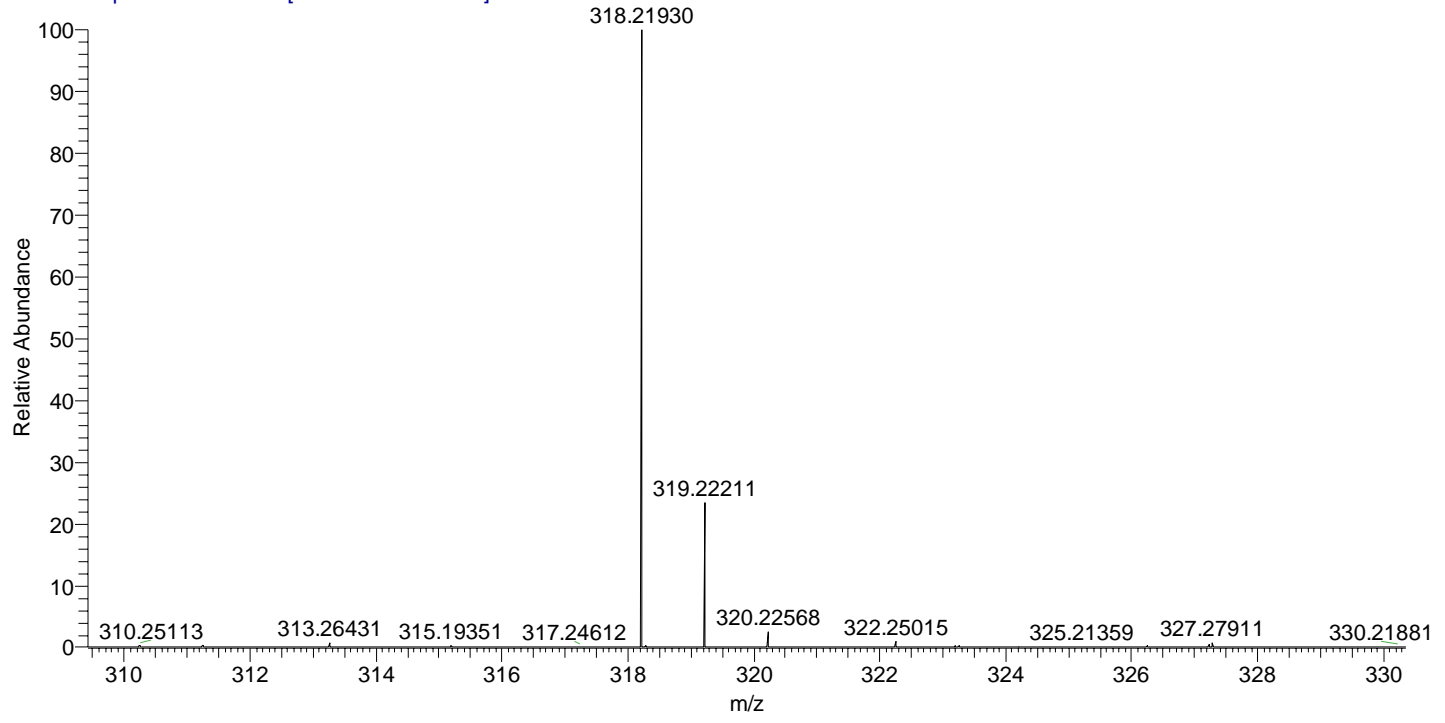


6-Methylbenzo[d]oxazole (5a. ${ }^{1} \mathrm{H}$ NMR $400 \mathrm{MHz}, \mathrm{CDCl}_{3} ;{ }^{13} \mathrm{C} \mathrm{NMR} 101 \mathrm{MHz}, \mathrm{CDCl}_{3}$ ).

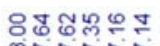

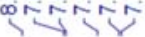
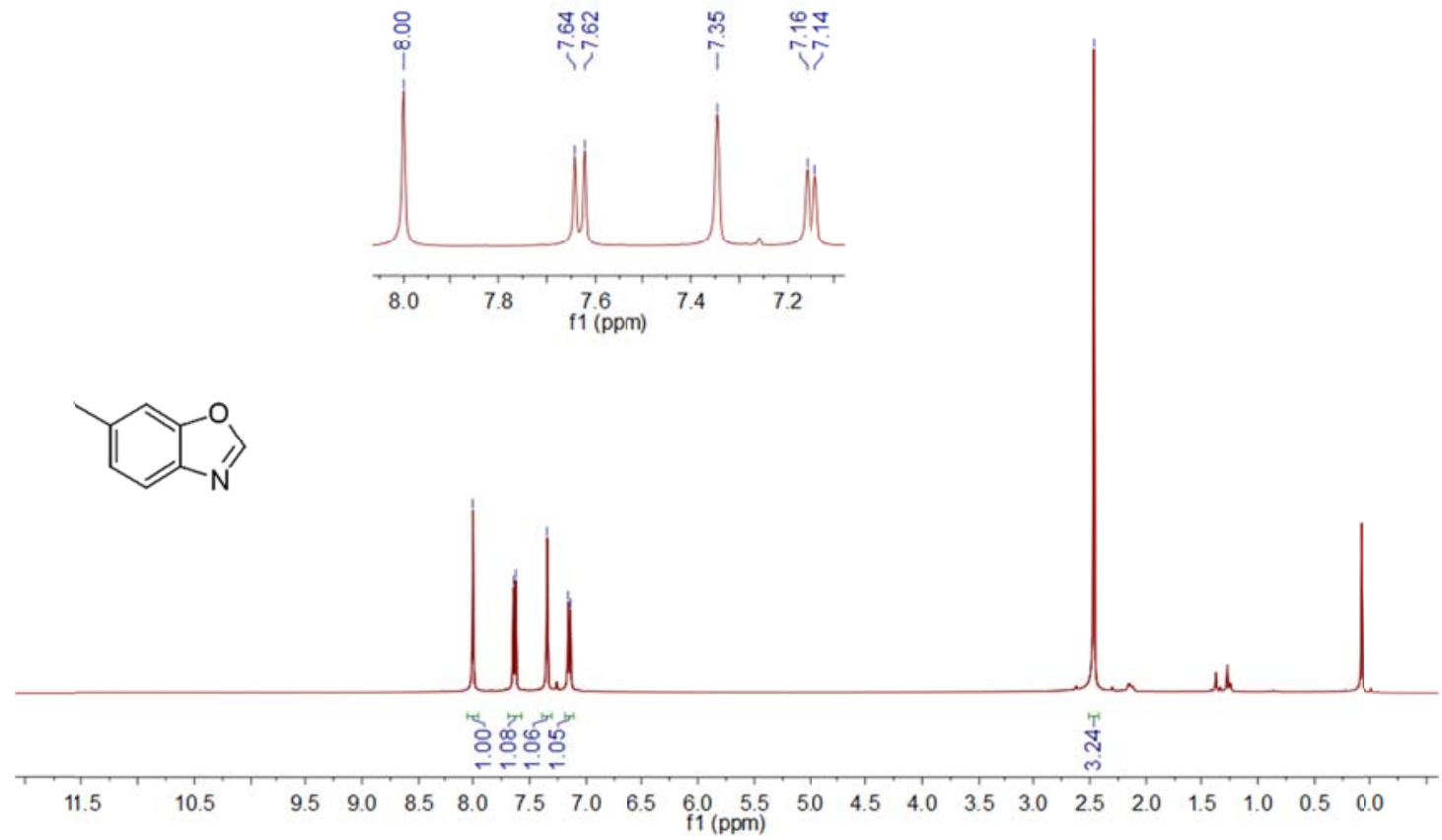

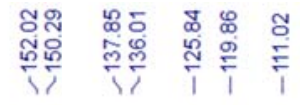

$\stackrel{\oplus}{\grave{N}}$

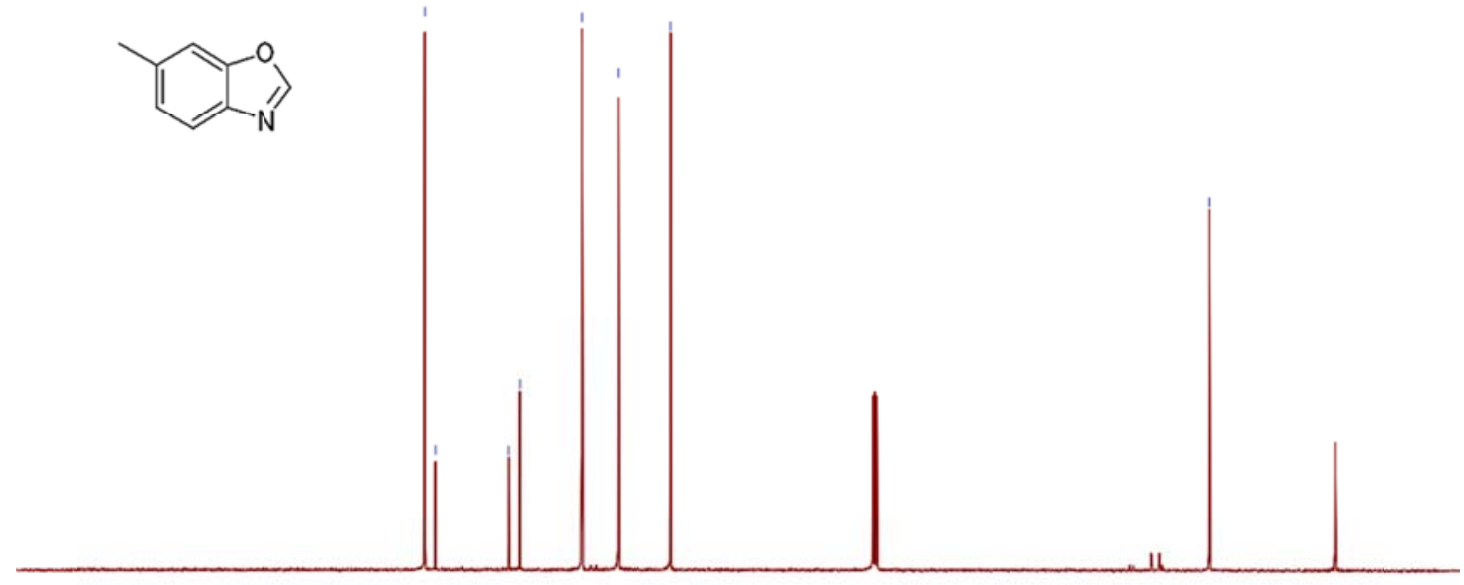

$\begin{array}{lllllllllllllllllllllll}210 & 200 & 190 & 180 & 170 & 160 & 150 & 140 & 130 & 120 & 110 & \begin{array}{l}100 \\ \mathrm{f} 1(\mathrm{ppm})\end{array} & 90 & 80 & 70 & 60 & 50 & 40 & 30 & 20 & 10 & 0 & -10\end{array}$ 
Benzo[d]oxazole (5b. ${ }^{1} \mathrm{H}$ NMR $400 \mathrm{MHz}, \mathrm{CDCl}_{3} ;{ }^{13} \mathrm{C} \mathrm{NMR} 101 \mathrm{MHz}, \mathrm{CDCl}_{3}$ ).

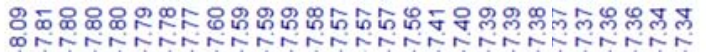

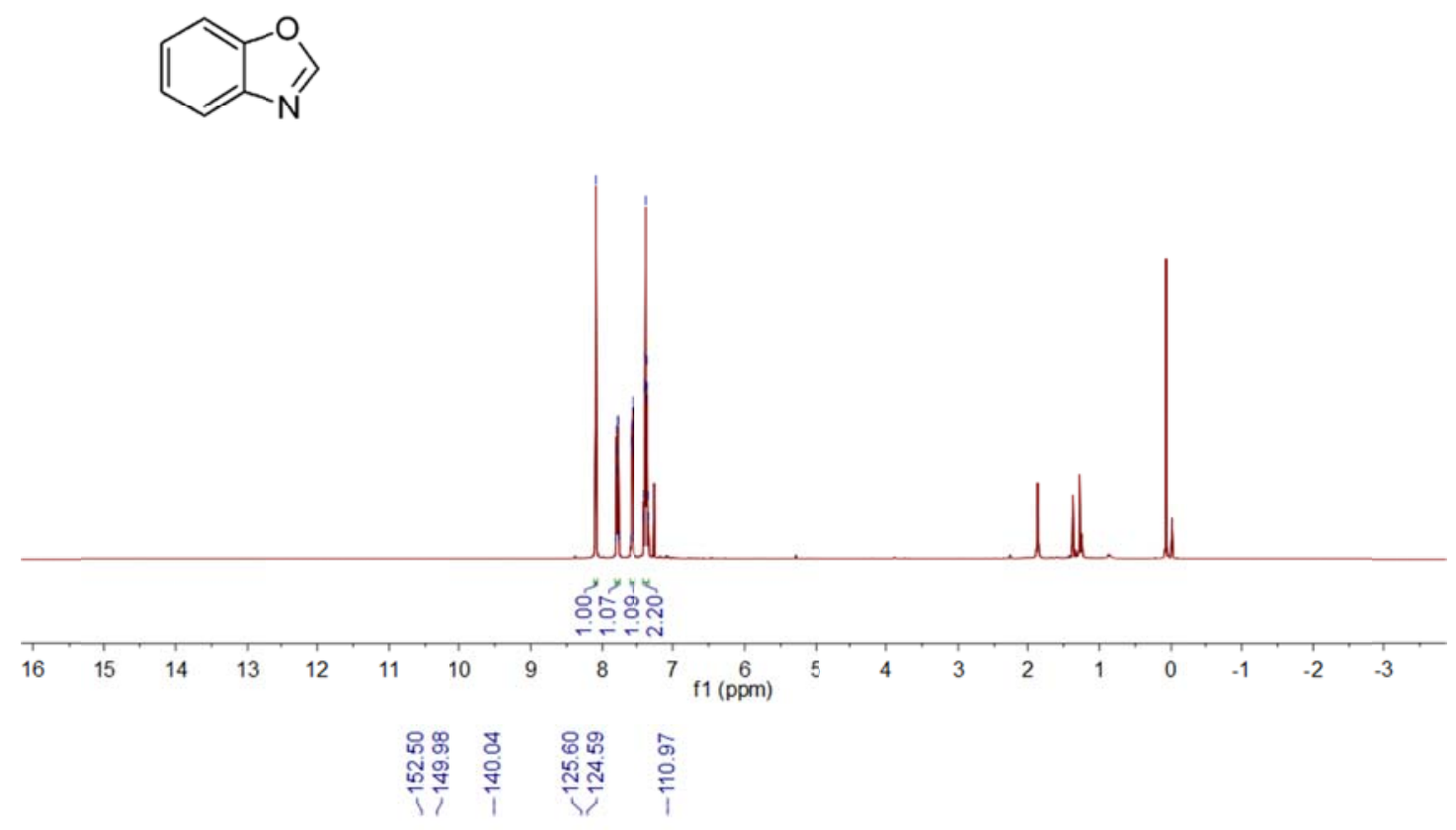

-

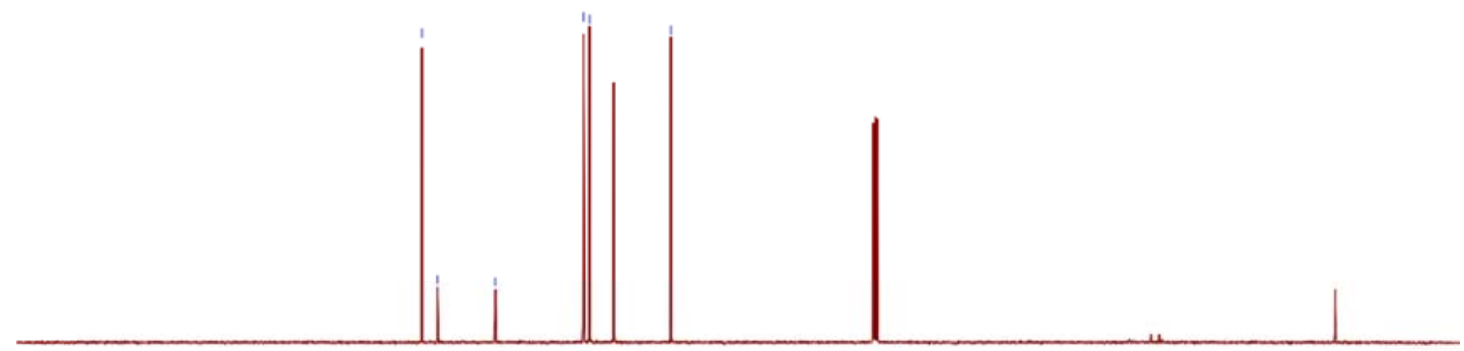

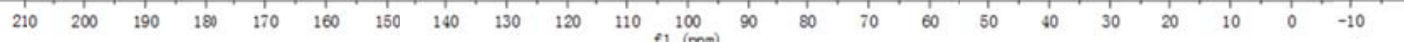


1-(Difluoromethyl)-1H-benzo[d]imidazole (5c. ${ }^{1} \mathrm{H}$ NMR $400 \mathrm{MHz}, \mathrm{CDC}_{13} ;{ }^{19} \mathrm{~F}$ NMR $376 \mathrm{MHz}$, $\mathrm{CDCl} 3 ;{ }^{13} \mathrm{C}$ NMR $\left.101 \mathrm{MHz}, \mathrm{CDCl} 3\right)$.
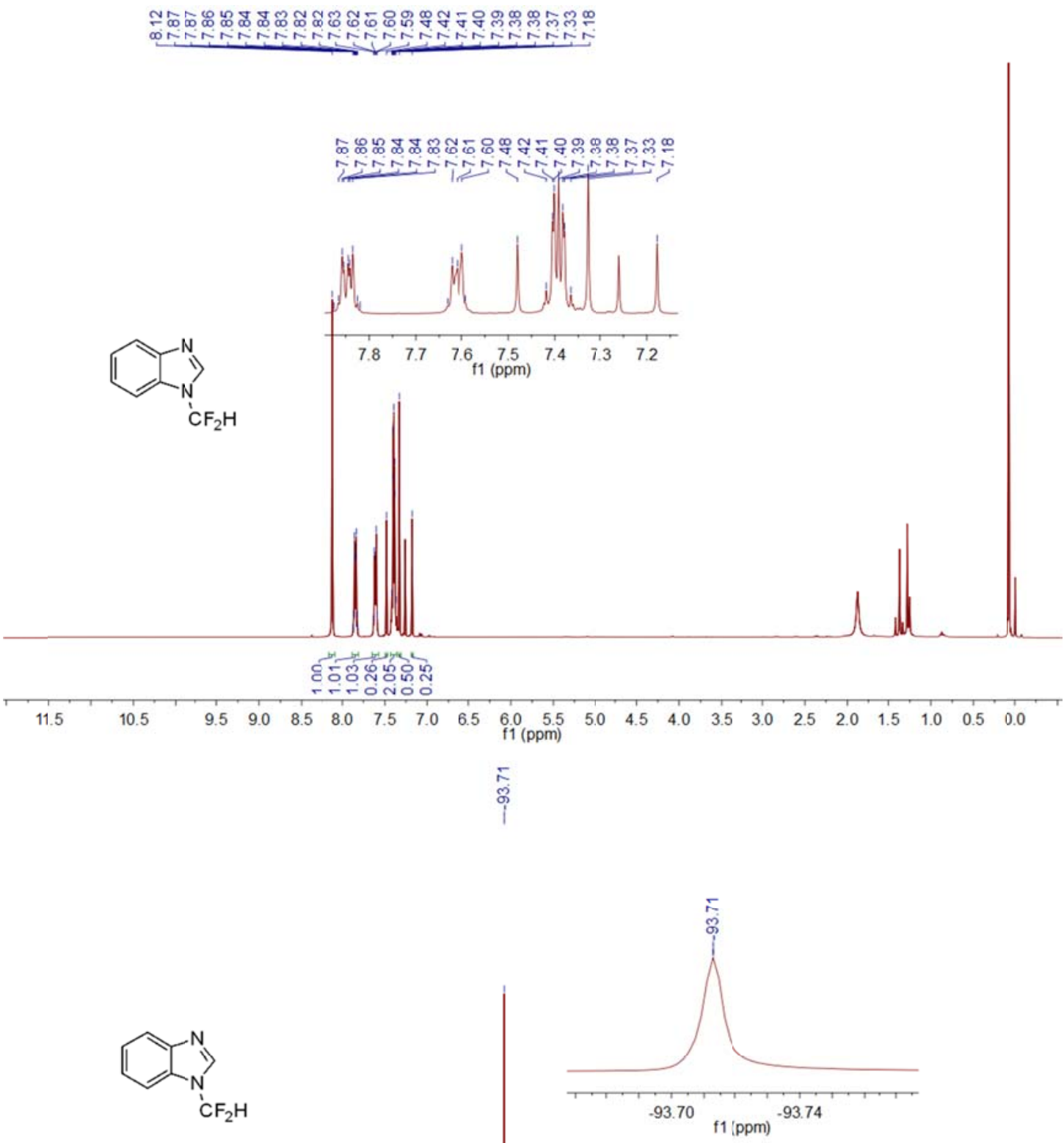

$\begin{array}{llllllllllllllll}10 & 0 & -10 & -20 & -30 & -40 & -50 & -60 & -70 & -80 & -90 \begin{array}{c}-100 \\ \mathrm{f} 1(\mathrm{ppm})\end{array} & -120 & -140 & -160 & -180 & -200\end{array}$ 

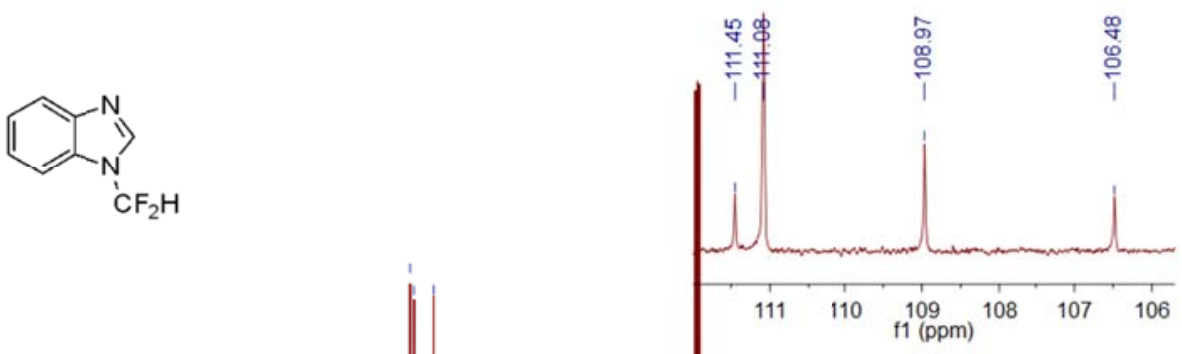

$\begin{array}{lllllllllllllllllllllll}210 & 200 & 190 & 180 & 170 & 160 & 150 & 140 & 130 & 120 & 110 & 100 & 90 & 80 & 70 & 60 & 50 & 40 & 30 & 20 & 10 & 0 & -10\end{array}$

4,5-diphenyl-1-tosyl-4,5-dihydro-1H-imidazole (5d. HRMS (ESI); ${ }^{1} \mathrm{H}$ NMR $400 \mathrm{MHz}, \mathrm{CDCl}_{3}$; ${ }^{13} \mathrm{C}$ NMR $101 \mathrm{MHz}, \mathrm{CDCl}_{3}$ ).

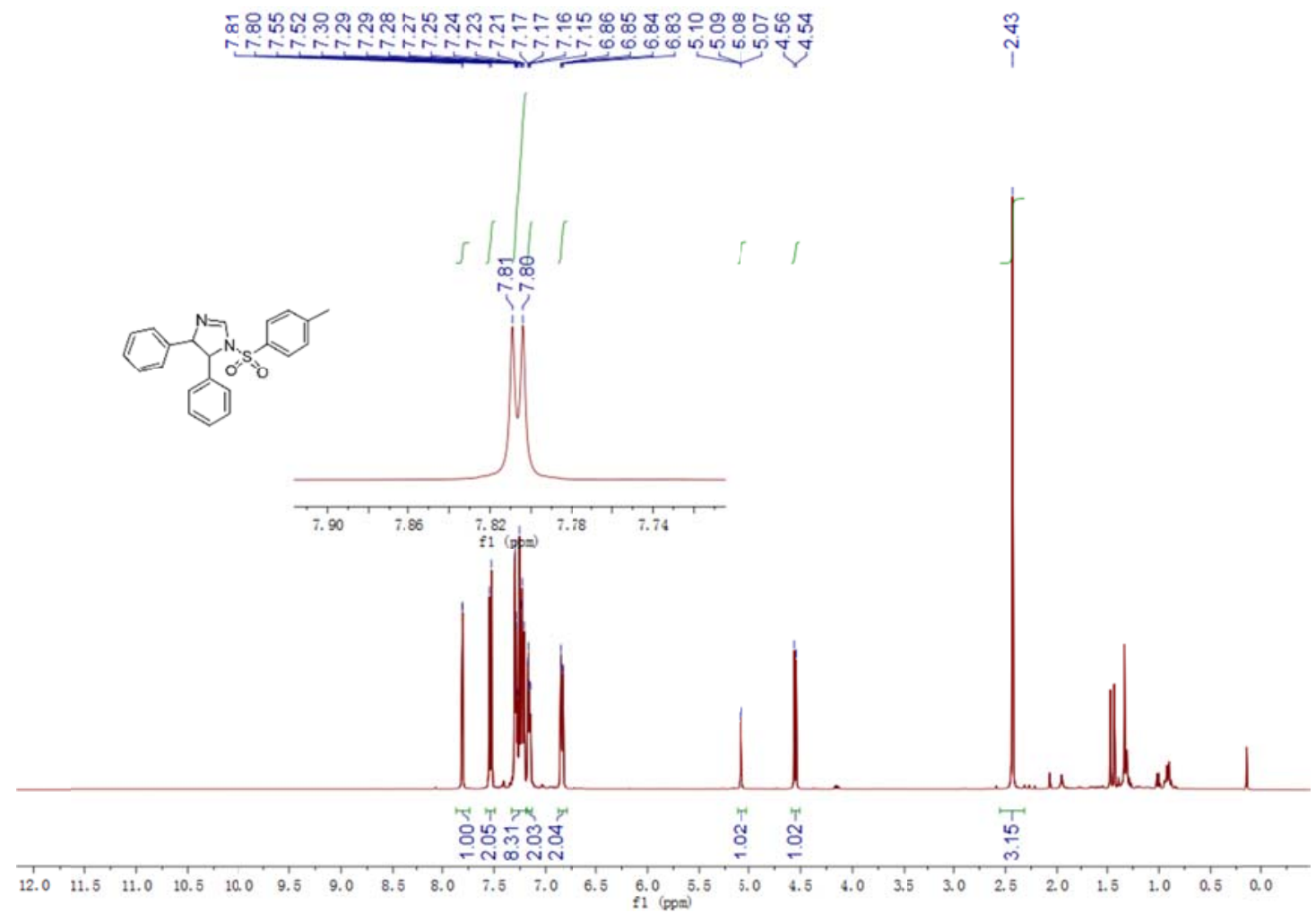




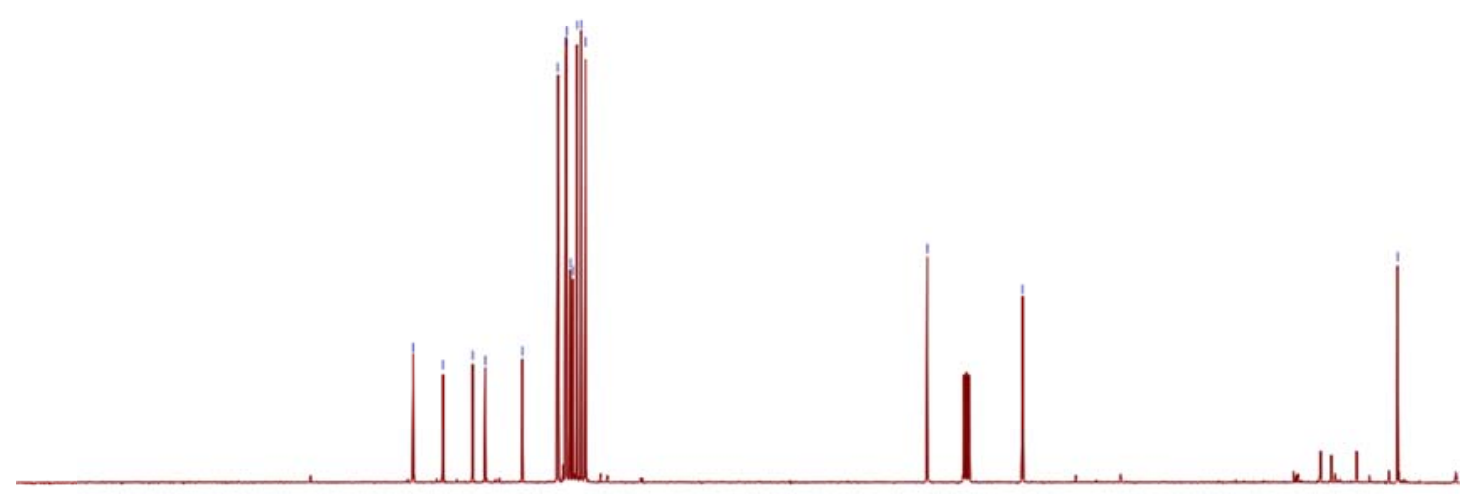

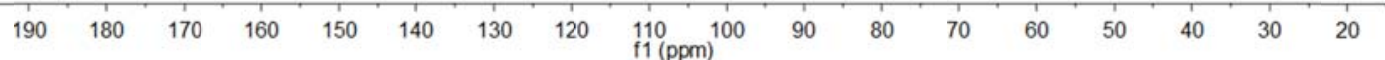

1-27 \#13 RT: 0.08 AV: 1 NL: $2.90 E 8$

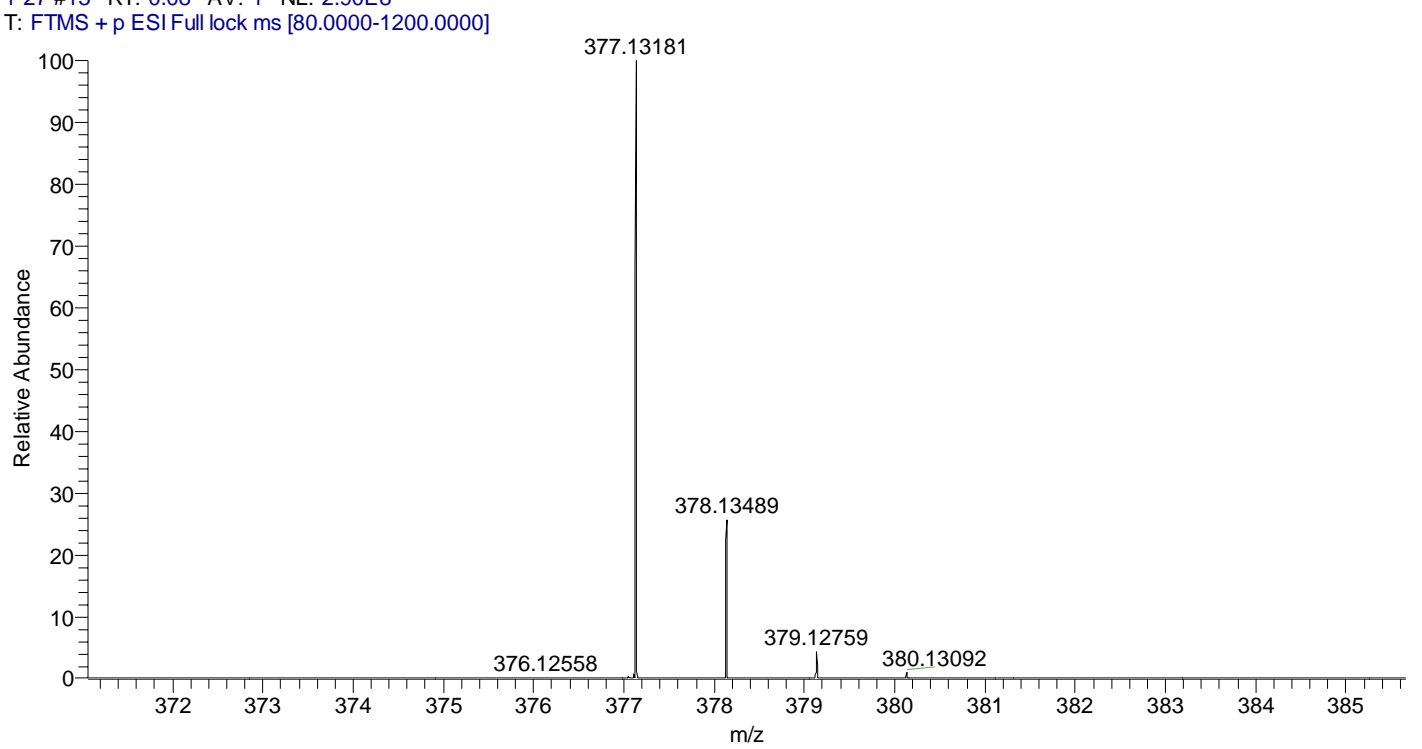


(E)-N-(2-(benzylamino)-1-(4-chlorophenyl)-2-oxoethyl)- $N$-phenylbut-2-enamide (9a. ${ }^{1} \mathrm{H}$ NMR

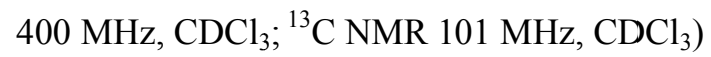
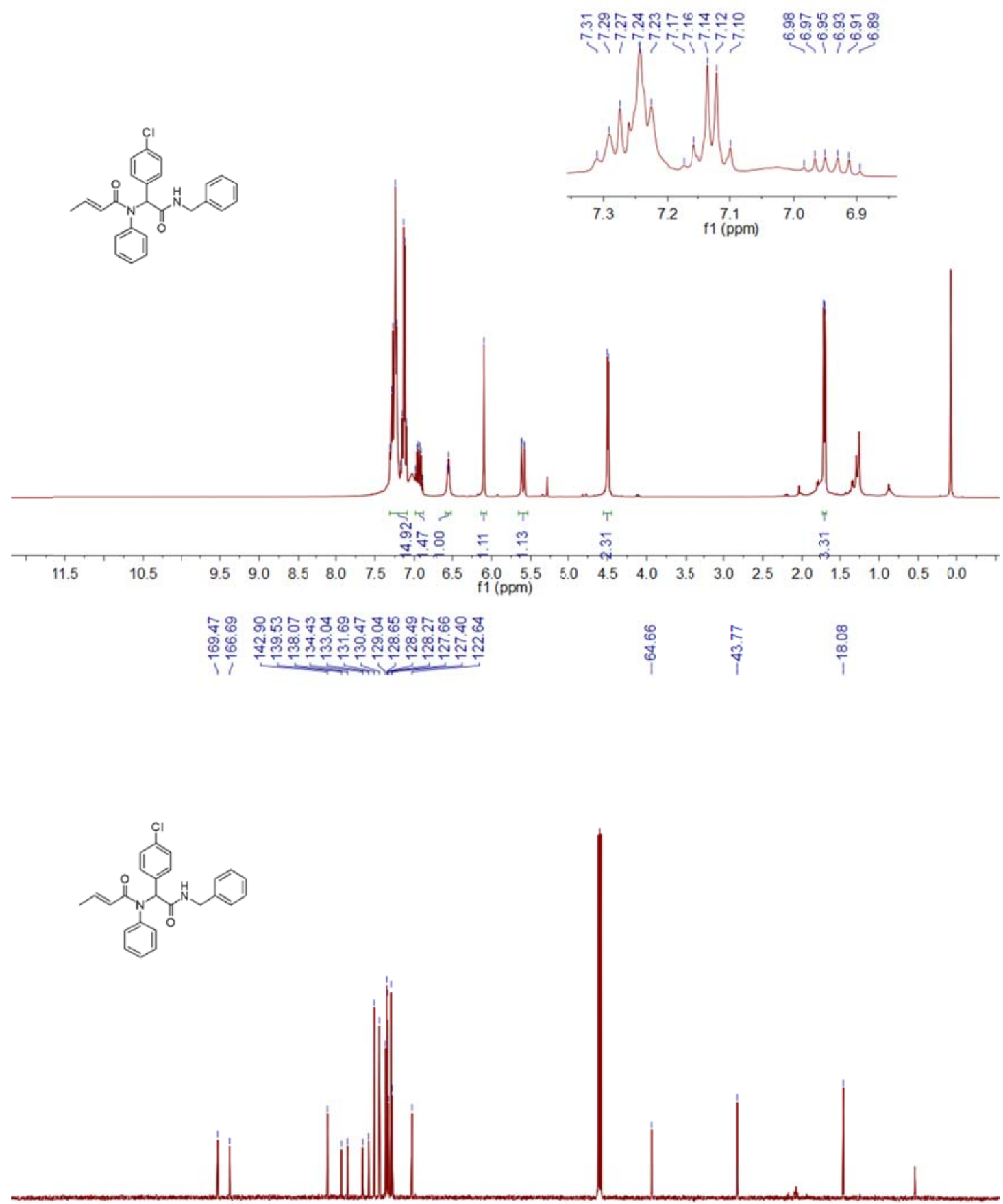

$\begin{array}{lllllllllllllllllllllll}210 & 200 & 190 & 180 & 170 & 160 & 150 & 140 & 130 & 120 & 110 \underset{\mathrm{f} 1(\mathrm{ppm})}{100} & 90 & 80 & 70 & 60 & 50 & 40 & 30 & 20 & 10 & 0 & -10\end{array}$ 
(E)- $N$-(2-oxo-1-phenyl-2-((2-phenylpropan-2-yl)amino)ethyl)- $N$-phenylbut-2-enamide $\left(9 b .{ }^{1} \mathrm{H}\right.$ $\mathrm{NMR} 400 \mathrm{MHz}, \mathrm{CDCl}_{3} ;{ }^{13} \mathrm{C} \mathrm{NMR} 101 \mathrm{MHz}, \mathrm{CDCl}_{3}$ ).

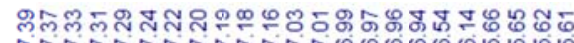

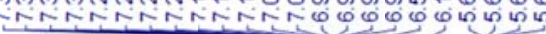
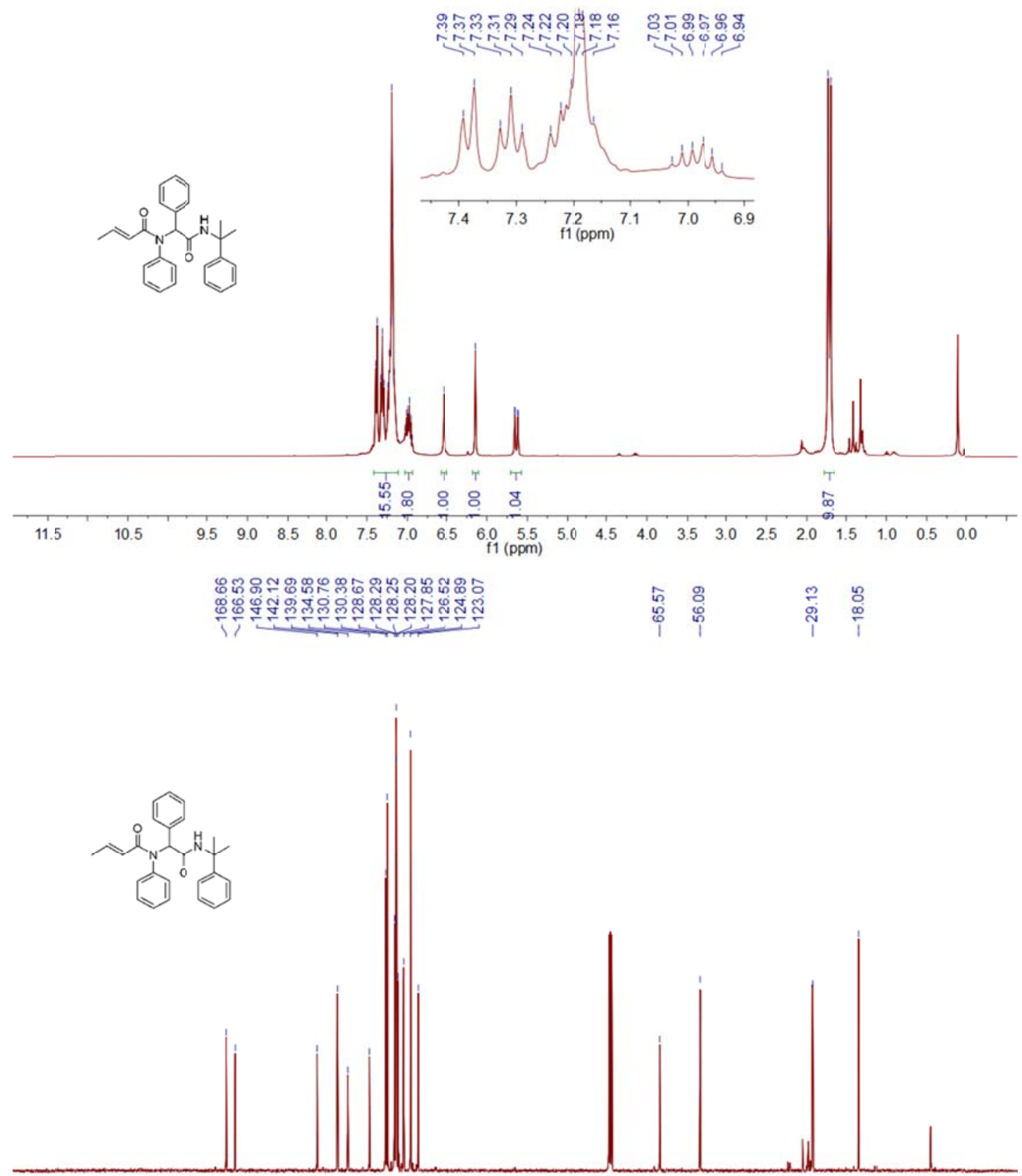

$\begin{array}{lllllllllllllllllllllll}210 & 200 & 190 & 180 & 170 & 160 & 150 & 140 & 130 & 120 & 110 & \begin{array}{c}100 \\ \mathrm{f} 1(\mathrm{ppm})\end{array} & 90 & 80 & 70 & 60 & 50 & 40 & 30 & 20 & 10 & 0 & -10\end{array}$ 
(E)-N-(1-(4-methoxyphenyl)-2-oxo-2-((2-phenylpropan-2-yl)amino)ethyl)- $N$-phenylbut-2-ena mide (9c. ${ }^{1} \mathrm{H}$ NMR $400 \mathrm{MHz}, \mathrm{CDCl}_{3} ;{ }^{13} \mathrm{C} \mathrm{NMR} 101 \mathrm{MHz}, \mathrm{CDCl}_{3}$ ).
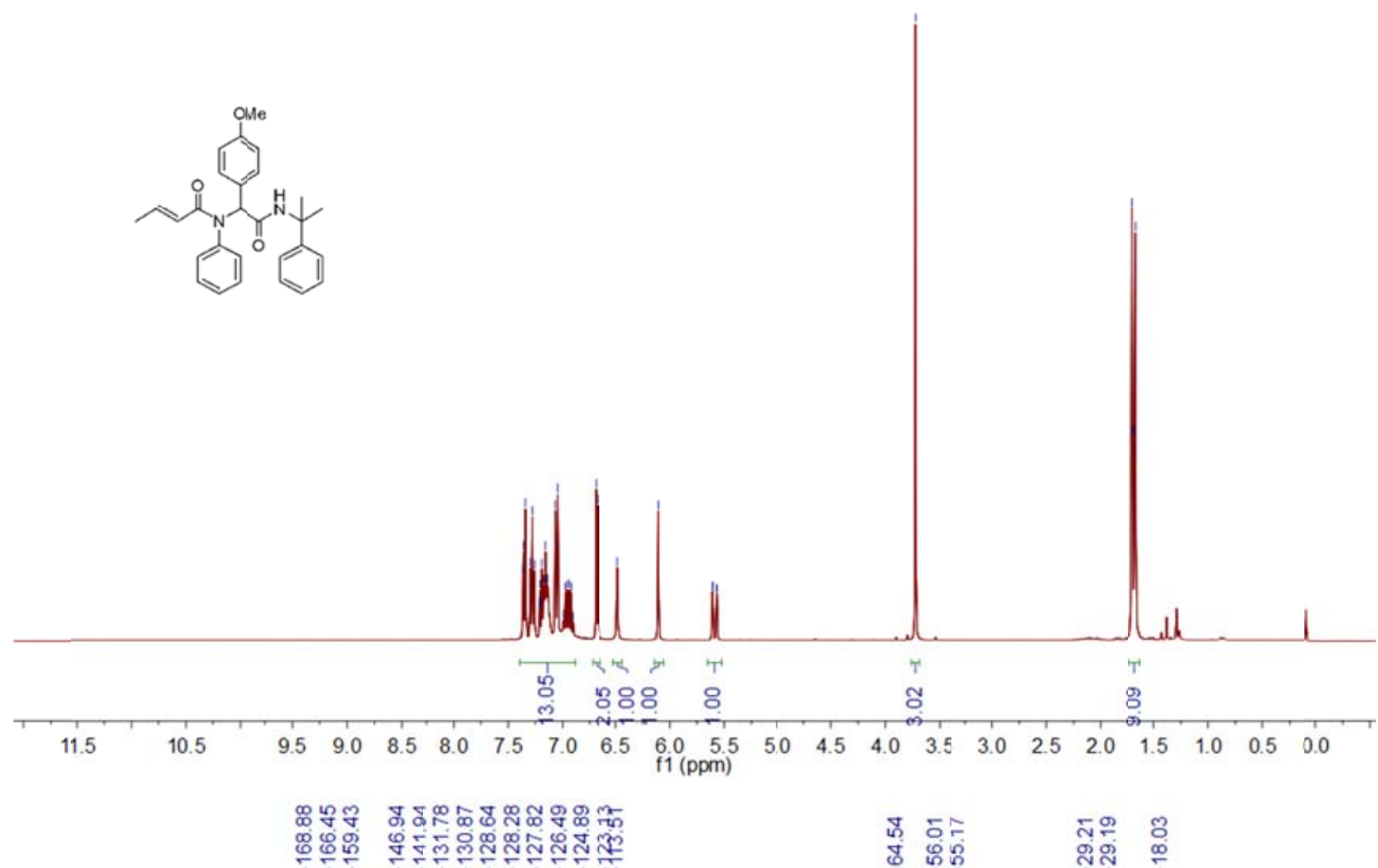

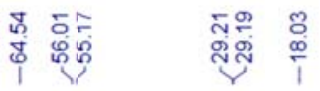

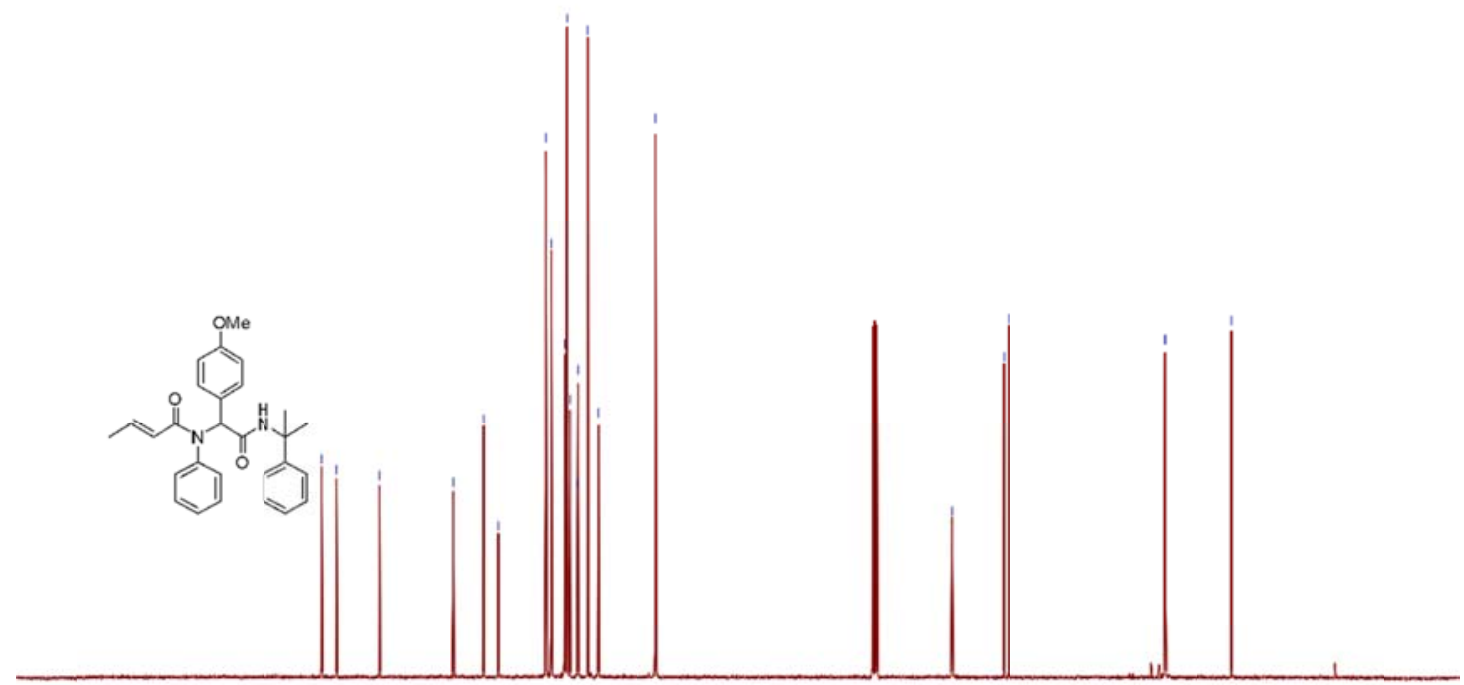

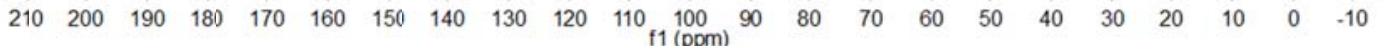


methyl (2-oxo-1-phenyl-2-((2-phenylpropan-2-yl)amino)ethyl)phenylalaninate (13a. HRMS (ESI)).

1-1 \#22 RT: $0.12 \quad$ AV: 1 NL: $2.42 E 9$

T: FTMS + p ESI Full ms [80.0000-1200.0000]

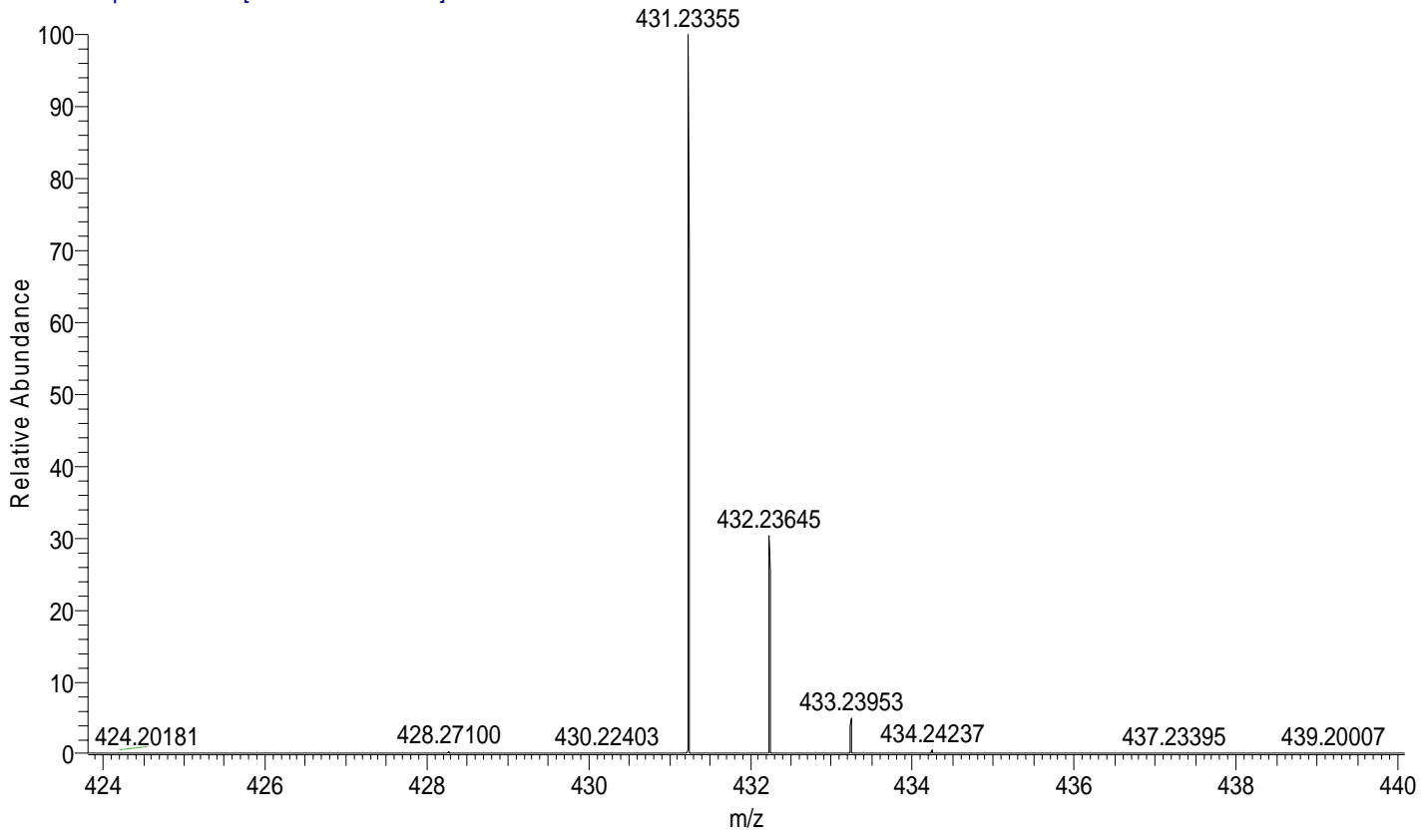

methyl

$N$-(2-oxo-1-phenyl-2-((2-phenylpropan-2-yl)amino)ethyl)- $N$-((2-oxo-1-phenyl-2-((2-phenylpr opan-2-yl)amino)ethyl)phenylalanyl)phenylalaninate (14a. HRMS (ESI))

1-1 \#22 RT: 0.12 AV: 1 NL: 8.71E6

T: FTMS + p ESI Full ms [80.0000-1200.0000]

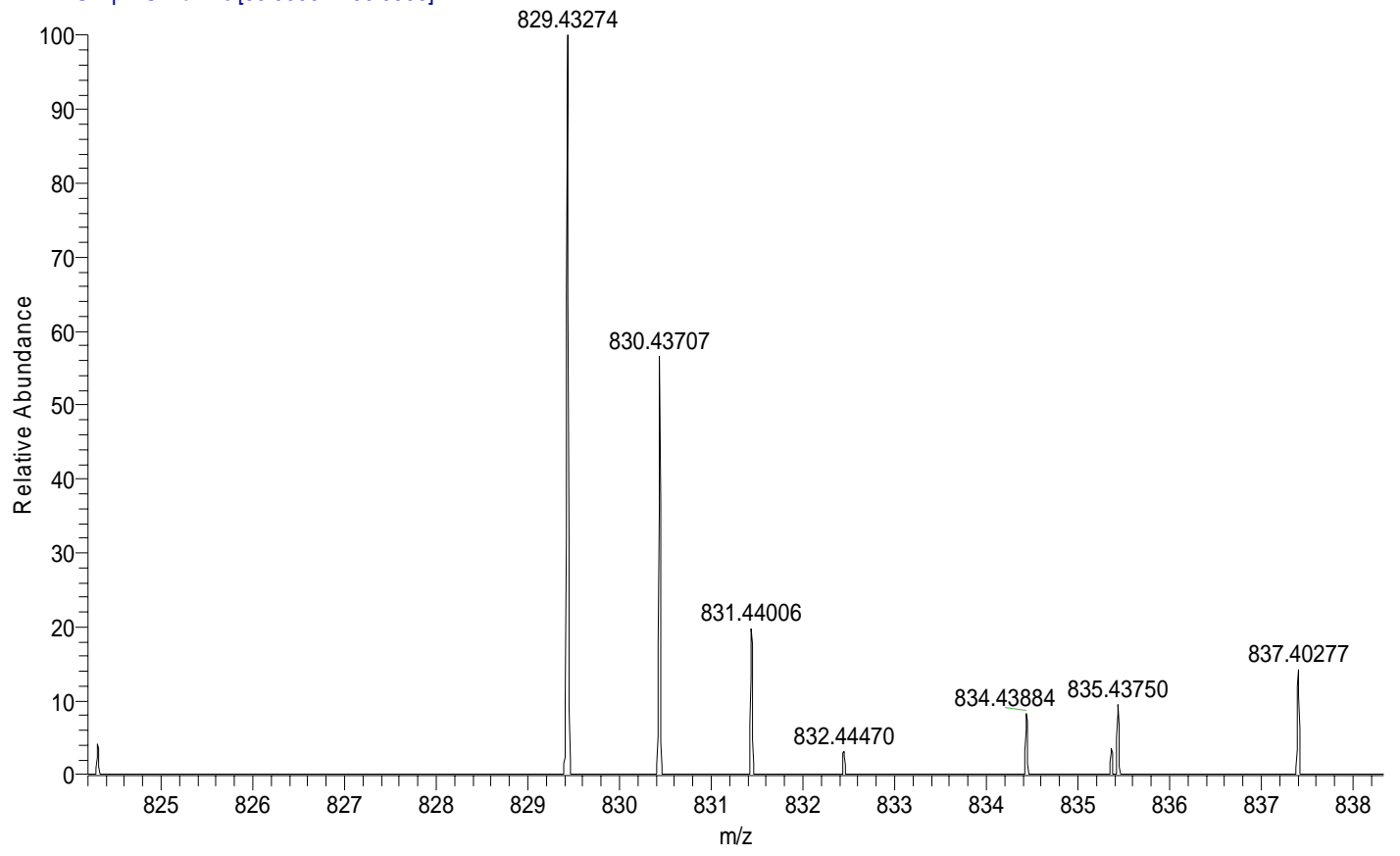


methyl (2-oxo-1-phenyl-2-((2-phenylpropan-2-yl)amino)ethyl)tryptophanate (13b. HRMS (ESI))

1-2 \#18 RT: 0.10 AV: 1 NL: 3.20E9

T: FTMS + p ESI Full lock ms [80.0000-1200.0000]

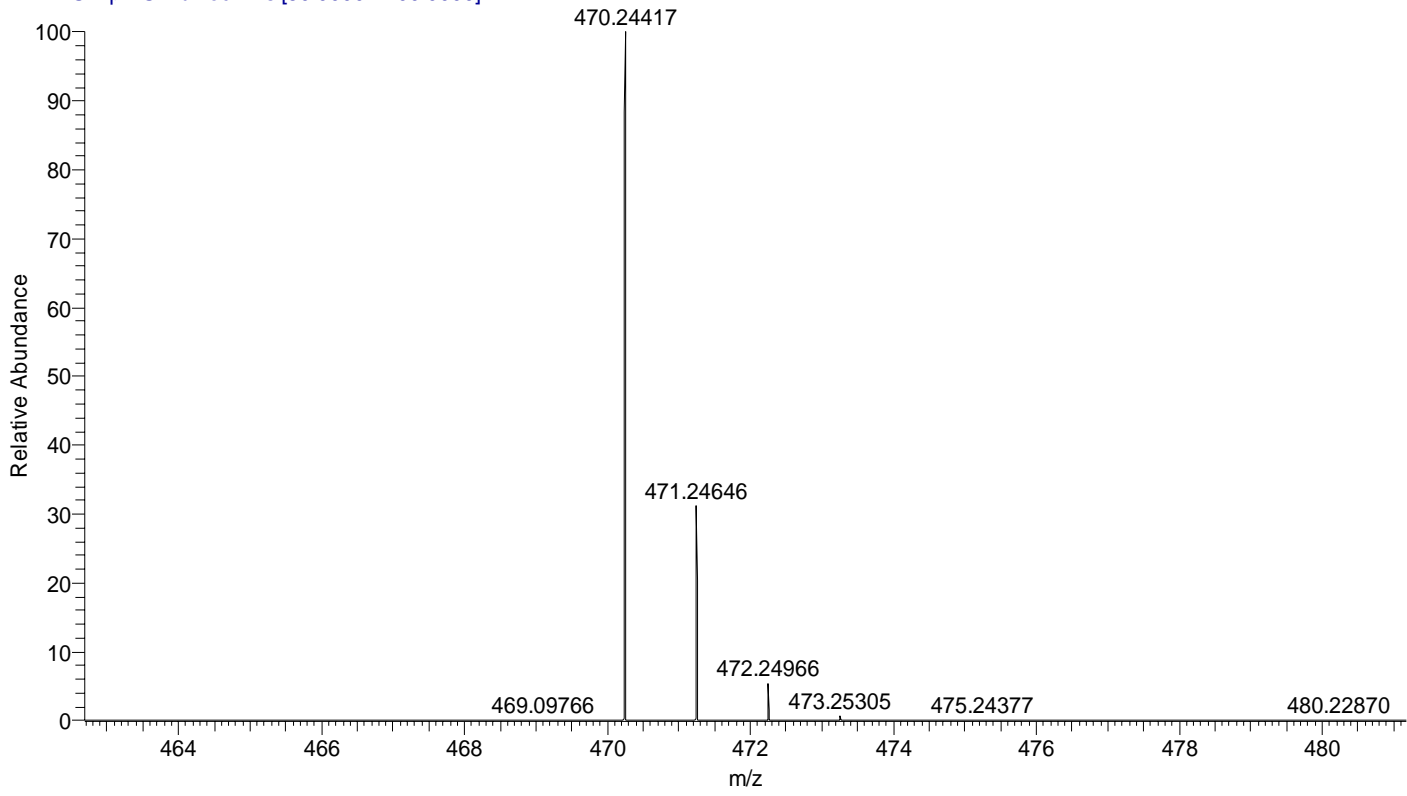

methyl

$N$-(2-oxo-1-phenyl-2-((2-phenylpropan-2-yl)amino)ethyl)- $N$-((2-oxo-1-phenyl-2-((2-phenylpr opan-2-yl)amino)ethyl)tryptophyl)tryptophanate (14b. HRMS (ESI))

1-2 \#18 RT: $0.10 \quad$ AV: 1 NL: $1.02 E 6$

T: FTMS + p ESI Full lock ms [80.0000-1200.0000]

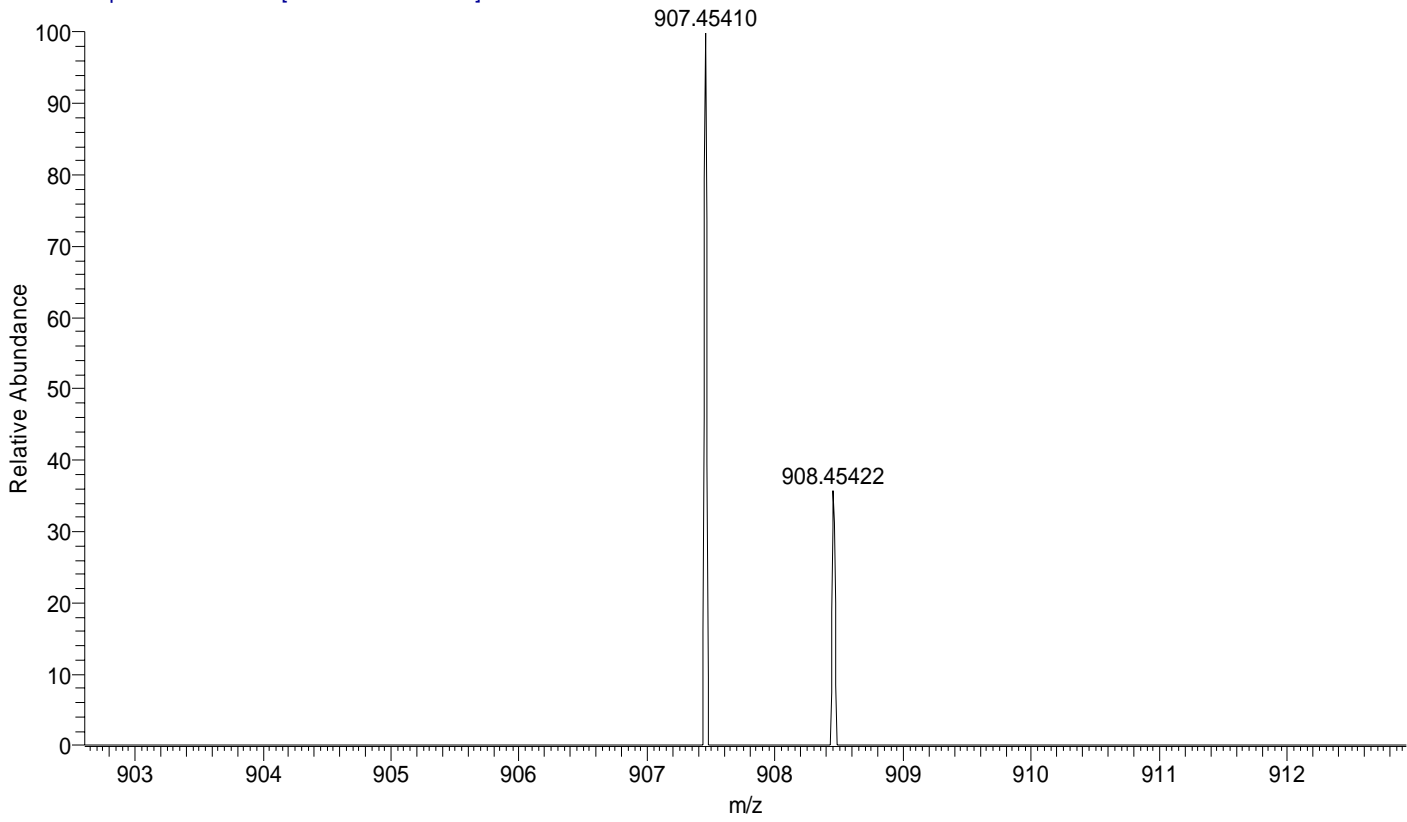

